\title{
Diversidad y conservación de los murciélagos de Loreto, Perú
}

\author{
Diversity and conservation of bats from Loreto, Peru
}

Laura Graham-Angeles* 1

https://orcid.org/0000-0003-1966-6534

laura.graham@unmsm.edu.pe

Pamela Sánchez-Vendizú ${ }^{1,2}$

https://orcid.org/0000-0002-3374-6031

p.sanchez.vendizu@gmail.com

Silvia Diaz ${ }^{1}$

https://orcid.org/0000-0002-9344-4991

silvia.diaz2@unmsm.edu.pe

Víctor Pacheco ${ }^{1,3}$

https://orcid.org/0000-0002-1005-135X

vpachecot@unmsm.edu.pe

\section{*Corresponding author}

1 Universidad Nacional Mayor de San Marcos, Museo de Historia Natural, Apartado 14-0434, Lima-15072, Perú.

2 Programa de Pos-Graduação em Zoología, Universidade Federal do Pará, Museu Paraense Emilio Goeldi, Brazil.

3 Universidad Nacional Mayor de San Marcos, Facultad de Ciencias Biológicas, Institutode Ciencias Biológicas "Antonio Raimondi", Lima, Perú.

\section{Citación}

Graham-Angeles L, Sánchez-Vendizú P, Diaz S, Víctor Pacheco V. 2021. Diversidad y conservación de los murciélagos de Loreto, Perú. Revista peruana de biología 28(especial): e21917 001- 056 (Diciembre 2021). doi: http://dx.doi.org/10.15381/rpb.v28iespecial.21917

\section{Presentado: $\quad 09 / 02 / 2021$

Aceptado: $\quad 10 / 08 / 2021$ \\ Publicado online: 30/12/2021}

Editor:

Leonardo Romero

\section{Resumen}

En este trabajo presentamos una lista actualizada de los murciélagos de Loreto basada en la revisión de bases de datos, colecciones científicas y literatura especializada. Encontramos que Loreto es el departamento con mayor diversidad de murciélagos del Perú, con 114 especies agrupadas en 7 familias (19.9\% de la mastofauna peruana), y que incluyen dos de las nueve especies endémicas para Perú: Micronycteris (Micronycteris) matses y Hsunycteris dashe. Además, se encontraron 444 localidades diferentes con registros de especímenes, principalmente ubicadas a lo largo de ríos. Las especies registradas en cuatro o menos localidades únicas fueron consideradas como raras, mientras que las especies registradas en 111 o más localidades únicas fueron consideradas comunes. Presentamos un mapa de las ecorregiones de Loreto con las localidades únicas, tanto históricas como contemporáneas, además de un mapa de densidad de localidades por cada $25 \mathrm{~km}^{2}$ donde se identifican 6 zonas de vacíos de información: 1) norte y centro de Putumayo 2) noroeste de Maynas y noreste de Loreto, 3) centro de Maynas, 4) centro-norte del Datem del Marañón, norte de Alto Amazonas y extremo occidental de Loreto, 5) centro-sur de Requena y extremo oriental de Ucayali y 6) extremo occidental de Ucayali. Finalmente presentamos mapas de distribución para cada especie aquí reportada para Loreto.

\section{Abstract}

In this work, an updated list of species of bats from Loreto is presented, this was elaborated using information of databases, zoological collections, and specialized literature. The results confirm Loreto as the most diverse department of Peru with 114 species of bats grouped into 7 families (19.9\% of the Peruvian mastofauna), which include two of the nine endemic species to Peru: Micronycteris (Micronycteris) matses and Hsunycteris dashe. Additionally, 444 different locations were found with specimen records, located mainly along rivers. Species recorded in four or less different locations are considered rare, while species recorded in 111 or more different locations are considered common. We present a map of the Loreto ecoregions with the different localities, both historical and contemporary, as well as a map of density of locations per $25 \mathrm{~km}^{2}$, which identifies 6 areas of information gaps: 1) North and middle Putumayo, 2) Northwest Maynas and northeast Loreto, 3) Middle Maynas, 4) North-middle Datem del Marañón, north Alto Amazonas and western end of Loreto, 5) South-middle Requena and eastern end of Ucayali and 6) Western end of Ucayali. Finally we present distribution maps for each species reported here for Loreto.

Palabras clave:

Amazonia; Distribución; Endemismo; Mammalia; Selva baja.

Keywords:

Amazonia; Distribution; Endemism; Mammalia; lowland forest. 


\section{Introducción}

El Perú se encuentra entre los cinco países a nivel mundial con mayor diversidad de mamíferos, estimándose que existen no menos de 573 especies de mamíferos a lo largo del territorio peruano (Pacheco et al. 2021); no obstante, se conoce poco sobre la diversidad de mamíferos a nivel de departamentos, contando solo con listas para Arequipa, Ucayali y Loreto (Zeballos et al. 2001, Quintana et al. 2009, Pitman et al. 2013). A pesar de que Loreto es el departamento más grande del Perú, ubicado en la Amazonia occidental y ocupa el $28.7 \%$ del territorio nacional con una extensión de 368851 km², el conocimiento de su fauna es escaso, y en particular la diversidad de murciélagos, perjudicando la correcta toma de decisiones en conservación y de desarrollo sostenible.

En la lista más reciente de mamíferos de Loreto presentada por Pitman et al. (2013) se estimaron 140 especies de murciélagos para este departamento, entre especies registradas (131) y esperadas (9). Las especies registradas estuvieron basadas en mapas de distribución de la IUCN y en la lista anotada de Pacheco et al. (2009), mientras que las especies potenciales fueron aquellas cuya distribución según la IUCN abarca el departamento de Loreto.

Debido a considerables cambios taxonómicos, descripción de especies nuevas, así como nuevos registros de especies, se hace necesaria la actualización de la lista presentada por Pitman et al. (2013). Con esta finalidad, el presente trabajo documenta la diversidad de murciélagos para el departamento de Loreto basado en la revisión de bases de datos de museos nacionales e internacionales y literatura especializada. Los resultados incluyen: 1) Una lista de especies, señalándose su presencia en las provincias, localidades, endemismo y categoría de conservación según la IUCN y el DS № 004-2014,2) un mapa de la ubicación de las localidades únicas, 3) un mapa de vacíos de información en base a la densidad de las localidades únicas y 4) mapas de distribución para cada especie distribuida en Loreto.

\section{Material y métodos}

Área de estudio. - El departamento de Loreto se localiza en el nororiente de Perú, aproximadamente entre los paralelos $0^{\circ} 2^{\prime} 5^{\prime \prime}-8^{\circ} 42^{\prime} 6^{\prime \prime} \mathrm{S}$ y los meridianos $77^{\circ} 47^{\prime} 14^{\prime \prime}$ $69^{\circ} 56^{\prime} 41^{\prime \prime}$ W. Con un área de $368851 \mathrm{~km}^{2}$ representa un tercio del territorio nacional, donde predomina la ecorregión del Bosque Húmedo Amazónico (BHA, Britto 2017). Su topografía es relativamente plana, que va de 70 a 220 m aprox., excepto en la región de la Cordillera Escalera que puede llegar a los 2000 m (Patterson \& López Wong 2014). Loreto presenta un clima cálido y muy húmedo con abundantes precipitaciones pluviales. Al oeste de Loreto, los ríos Morona, Pastaza, Tigre y Huallaga son grandes afluentes del río Marañón, el cual se une con el río Ucayali cerca a Nauta para formar el gran río Amazonas. Al este de Loreto, el río Curaray es afluente del río Napo, que a su vez vierte sus aguas al río Amazonas cerca de Francisco de Orellana. El río Putumayo limita a Loreto con Colombia, pero desemboca en el río Amazonas ya en territorio brasileño.
Según el SERNANP, en el departamento de Loreto encontramos 11 Áreas Naturales Protegidas por el Estado bajo el Sistema Nacional de Áreas Naturales Protegidas por el Estado (SINANPE), divididas en 4 Parques Nacionales, 4 Reservas Nacionales, 2 Reservas Comunales y una Zona Reservada, además de 34 Áreas Naturales Protegidas regionalmente o a nivel privado divididas en 4 Áreas de Conservación Regional y 30 Áreas de Conservación Privadas (ACP). Las 11 áreas dirigidas bajo el SINANPE suman un aproximado de $71988.12 \mathrm{~km}^{2}$ solo en Loreto, sin contar las áreas compartidas con otros departamentos.

Datos y análisis. - Para elaborar la lista de especies se tomó como punto de partida los artículos de Pacheco et al. (2018, 2021). Además se consultó literatura especializada, bases de datos disponibles en portales especializados (GBIF, VERNET) que incluyen los repositorios más importantes como las colecciones del American Museum of $\mathrm{Na}$ tural History, New York (AMNH), Field Museum of Natural History, Chicago (FMNH), Louisiana State University, Museum of Zoology (LSUMZ), Museum of Texas Tech University (MTTU), Museum of Comparative Zoology, Massachusetts (MCZ), Natural History Museum of Kansas University (KU), Royal Ontario Museum, Toronto (ROM), Texas A\&M Biodiversity Research and Teaching Collections (TCWC) y el Smithsonian National Museum of Natural History, Washington, DC (USNM). A nivel nacional, se incluyó la base de datos de la colección del Museo de Historia Natural, Universidad Nacional Mayor de San Marcos (MUSM). Para la nomenclatura y arreglo taxonómico se siguió a Gardner (2008) y a Pacheco et al. (2009), agregándose cambios taxonómicos recientes. Para murciélagos, estos cambios incluyen a Velazco y Simmons (2011), Parlos et al. (2014), Hurtado y Pacheco (2014), Baker et al. (2016), Velazco y Patterson (2014), Velazco et al. (2017), Basantes et al. (2020) y Velazco et al. (2021).

De las bases de datos y las publicaciones consultadas, se tomaron los siguientes datos para cada registro: nombre científico, nombre común, tipo de registro (observación directa o colecta), museo o instituto donde se depositó la muestra, número de catálogo (si fue colecta), referencia bibliográfica, nombre de la localidad de colecta y coordenadas geográficas.

Se consideraron como localidades únicas todos aquellos puntos que tenían el mismo nombre de la localidad y coordenadas similares, pero cuyos registros fueron obtenidos en una misma campaña (fechas cercanas). De tratarse de coordenadas tomadas por un mismo colector en distintas redes con una distancia de menos de $1 \mathrm{~km}$ entre ellas, se tomó la coordenada del punto central como representativa para esa localidad; pero de ser la distancia mayor, se separaron con una letra al final del nombre (por ejemplo, Quebrada Blanco A, Quebrada Blanco B). En las localidades sin coordenadas, éstas fueron estimadas de publicaciones como Stephens y Traylor (1983), Hershkovitz $(1983,1987)$, Valqui (2001), Willey (2010) o Patton et al. (2015). En caso de no haber referencia para las coordenadas, éstas se colocaron en base a otros registros del mismo colector $\mathrm{y} / \mathrm{o}$ fechas cercanas 
y/o mismo nombre de localidad. Además, especialistas en murciélagos amazónicos ayudaron a confirmar la presencia de las especies en tales localidades.

El estado de conservación de las especies está basado en la lista roja de la Unión Internacional para la Conservación de la Naturaleza (IUCN, https://www.iucnredlist. org/) y la legislación peruana (D.S. 004-2014). La información referente a nivel de endemismo se basó en Pacheco et al. (2009) y se actualizó con publicaciones científicas recientes (Mantilla-Meluk et al. 2009, Regalado \& Albuja 2012, Medina et al. 2014, Velazco et al. 2017, Moratelli et al. 2019, Málaga et al. 2020, Morales-Martínez et al. 2020). De tal manera que, las especies endémicas para el departamento de Loreto (que a su vez son endémicas para el país) van señaladas por una $\mathrm{R}$ (Tabla 1). Consideramos una especie como rara cuando estaba representada en cuatro localidades únicas o menos $(\leq 1 \%)$ y común cuando se presenta en al menos 111 localidades únicas $(\geq 25 \%)$.

Los mapas fueron elaborados con el programa ArcMap v.10.3. El primero (Fig 1) muestra la distribución de las localidades únicas en las diferentes ecorregiones según Britto (2017) señalando las localidades con registros considerados históricos, que van desde el año 1912 al 2000 y registros considerados contemporáneos, que van desde el 2001 hasta el 2018. El segundo mapa (Fig 2) muestra la densidad de localidades considerando celdas de $25 \mathrm{~km}^{2}$, exponiendo así las áreas con pocas o ninguna localidad muestreada, denominadas 'vacíos de información'.

\section{Resultados}

Diversidad y localidades.- A partir de las revisiones, el recuento de murciélagos para Loreto es de 114 especies, distribuidas en siete familias, 12 subfamilias y 53 géneros (Tabla 1), representando el 19.9\% del total de mamíferos registrados para Perú (573 especies, Pacheco et al. 2021), cerca del 44\% de mamíferos registrados para Loreto (261 spp.) y el $60 \%$ del total de murciélagos registrados para el país (189 especies, Pacheco et al. 2021), confirmando así la alta diversidad de este grupo de mamíferos existentes en la selva baja (Pacheco 2002) (Tabla 1).

Se encontraron 444 localidades únicas con registros de murciélagos, distribuidas en 8 provincias de Loreto, las cuales se encuentran en mayor cantidad a lo largo de los principales ríos y ciudades (Fig. 1, Anexo 1). Las referencias que soportan estas localidades para los registros de murciélagos se encuentran en el Anexo 2, mientras que los mapas de distribución de las 114 especies listadas se encuentran en el Anexo 3.

Maynas es la provincia con mayor cantidad de localidades únicas (283), mientras que las que tienen menos localidades muestreadas son Mariscal Ramón Castilla (12), Alto Amazonas (8) y Putumayo (8). De forma similar, las provincias que tienen la mayor diversidad de murciélagos son Maynas (98 especies) y Requena (90 especies), mientras que las de menor diversidad son Alto Amazonas y Putumayo, donde se registran 33 y 25 especies, respectivamente.

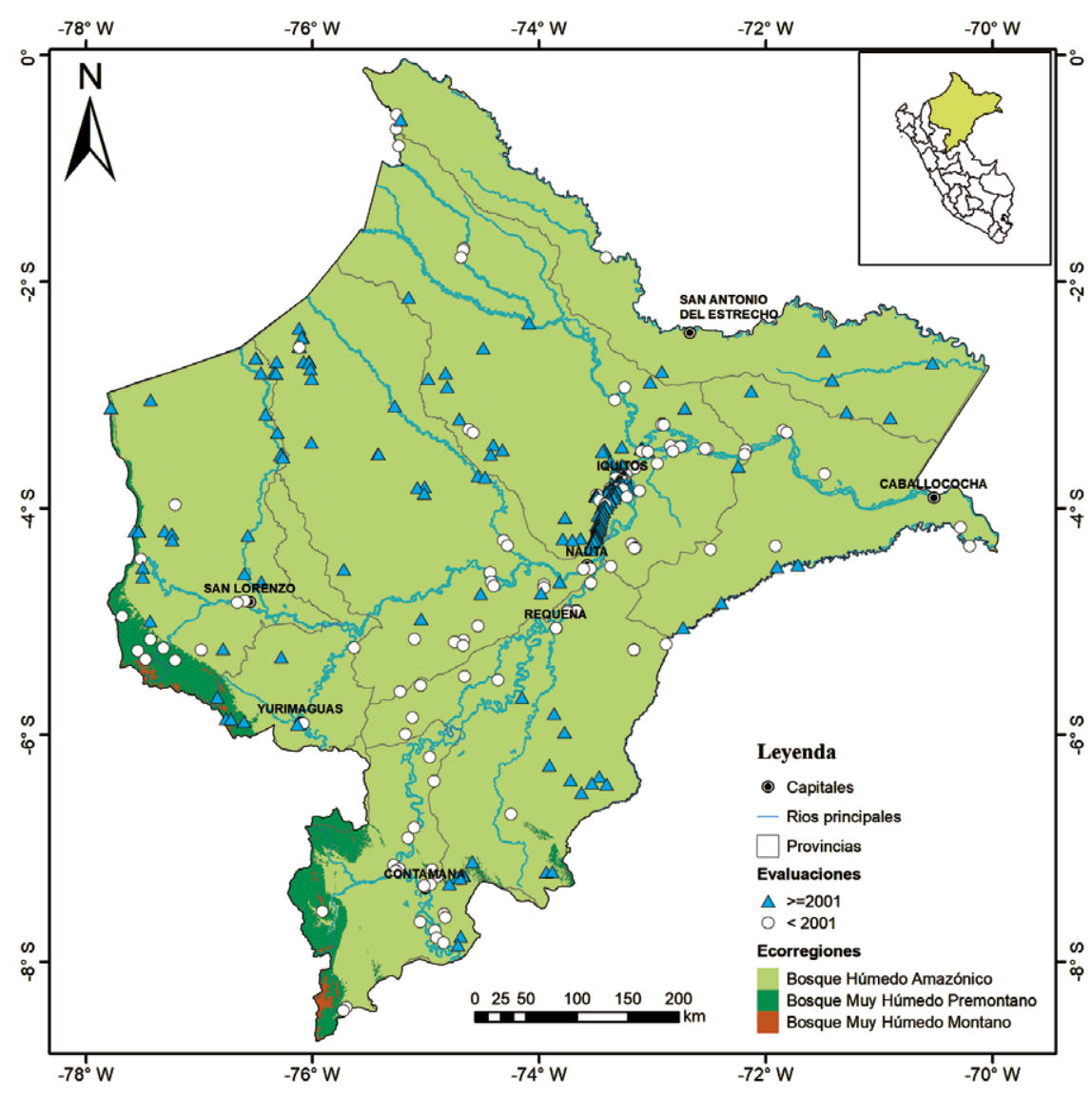

Figura 1. Mapa de localidades únicas históricas $(<2001)$ y contemporáneas $(>=2001)$ de especies de murciélagos por ecorregiones sensu Britto (2017) para el departamento de Loreto, Perú. 
Así mismo, siguiendo las ecorregiones propuestas por Britto (2017), 432 localidades se ubican en Bosque Húmedo Amazónico (BHA), 12 localidades, en Bosque Muy Húmedo Premontano (BMHP) y ninguna localidad se encontró en el Bosque Muy Húmedo Montano (BMHM).

Especies Endémicas.- El Perú tiene nueve especies endémicas del orden Chiroptera (Pacheco et al. 2021) y de éstas, sólo dos se han reportado para Loreto: Hsunycteris dashe y Micronycteris (Micronycteris) matses. Actualmente, la información referente a estas especies es escasa y solo han sido reportadas en dos localidades diferentes en la provincia de Requena (Tabla 1).

Conservación y rareza de especies.- Reportamos 78 especies protegidas en alguna de las Áreas Naturales Protegidas de Loreto y entre éstas, en las que mayor diversidad de murciélagos se han registrado son el Parque Nacional Sierra del Divisor (PNSD, 52 especies), la Reserva Nacional Allpahuayo Mishana (RNAM, 45 especies) y la Reserva Nacional Pacaya Samiria (RNPS, 42), mientras que las que poseen menor diversidad son la Zona Reservada de Santiago Comaina (ZRSC) y el Parque Nacional Cordillera Azul (PNCAZ), contando ambas con 9 especies en el territorio de la ANP correspondiente a Loreto.

No se registraron especies protegidas por categorías de amenaza (CR, EN, VU) para Loreto; sin embargo, la IUCN lista una especie en situación Casi Amenazada
(NT): Vampyrum spectrum, además de 100 especies en Preocupación Menor (LC) y 9 en Datos insuficientes (DD) (Tabla 1). Mientras que la Legislación Peruana, lista a dos especies con Datos insuficientes (DD): $\mathrm{Mi}$ cronycteris (Micronycteris) matses y Sphaeronycteris toxophyllum (Tabla 1).

Por otro lado, basados en el número de localidades en donde han sido registradas las especies, encontramos que ocho especies son comunes, con Carollia perspicillata y Artibeus planirostris liderando esta lista, mientras que las más raras son 33, dentro de las cuales están Anoura aequatoris, Artibeus bogotensis, Diclidurus albus, D. isabella, Eumops maurus, Lonchorhina aurita, Sturnira oporaphilum y Thyroptera wynneae, todas estas con solo un registro por especie para todo el departamento (Tabla 1).

Vacíos de Información. - La región de Loreto presenta algunas zonas con muy pocos o ningún registro de murciélagos, y son consideradas aquí como Vacíos de Información (Fig. 2), resaltadas en seis zonas de norte a sur: 1) Norte y centro de Putumayo, 2) Noroeste de Maynas y noreste de Loreto, 3) Centro de Maynas, 4) Centronorte del Datem del Marañón, norte de Alto Amazonas y extremo occidental de Loreto, 5) Centro-sur de Requena y extremo oriental de Ucayali y 6) Extremo occidental de Ucayali. Por lo que se sugiere dar prioridad a estas zonas en estudios de diversidad y conservación.

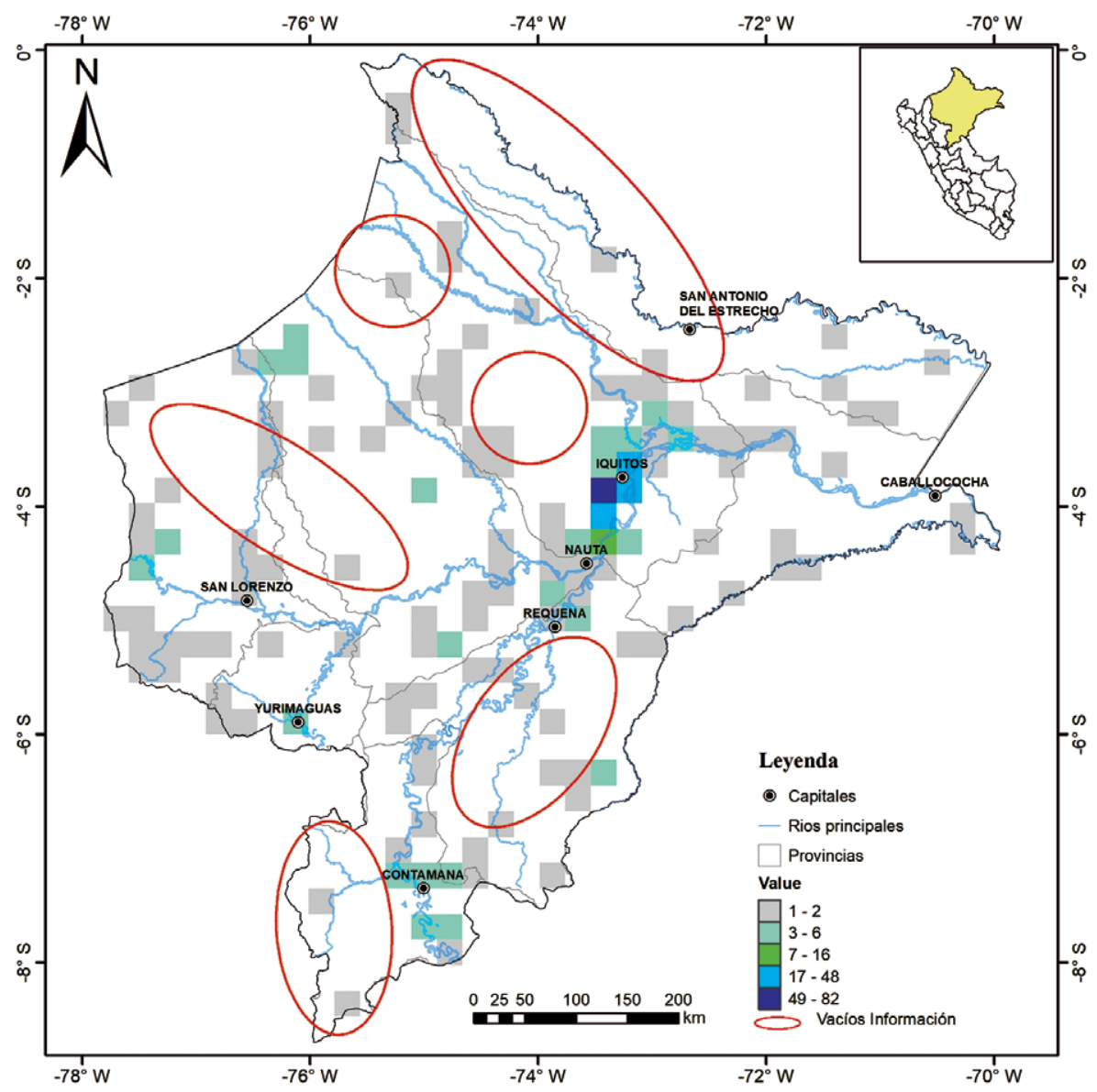

Figura 2. Mapa de densidad de localidades únicas registradas para las especies de murciélagos en el departamento de Loreto, Perú y sus vacíos de información. 


\section{Discusión}

Diversidad. - Este trabajo reúne información de diversas fuentes para estimar la riqueza de especies de murciélagos y su distribución geográfica dentro del departamento de Loreto, y logra compilar alrededor de 11000 registros basados en especímenes preservados en colecciones. Producto de esta compilación de datos, se elaboró un mapa con las localidades (únicas) donde se han registrado las 114 especies de murciélagos transpuestas sobre las 3 ecorregiones que presenta Loreto (Britto 2017). Se espera que esta información contribuya a la toma de decisiones en distintos niveles, como monitoreo de especies, sostenibilidad, conservación, estudios sobre enfermedades zoonóticas, entre otros.

Para confirmar la identificación de las especies, se consultaron con curadores y revisaron publicaciones, entre otras actividades. No obstante, en los casos de existir incertidumbre taxonómica respecto de algún registro, se recurrieron a las fuentes originales (por ejemplo, ejemplares depositados en Museos de Historia Natural), las cuales son listadas en el Anexo 2. En ese sentido, este trabajo se fundamenta en la trazabilidad de la información y facilita la ubicación de ejemplares y fuentes bibliográficas de donde se obtuvo la información y que permitirá a otras investigaciones su revisión.

De las 140 especies estimadas por Pitman et al. (2013), incluimos 101 especies en este trabajo dado que 39 no cuentan con especímenes que soportan su presencia en el departamento de Loreto. De estas 39 especies, 32 se han registrado en los departamentos contiguos de Ucayali, San Martín y Amazonas (Pacheco \& Patterson 1992, Solari et al. 2001, Pacheco 2003, Handley \& Gardner 2008, Davis \& Gardner 2008, Quintana et al. 2009, Rengifo et al. 2011, Tavares et al. 2014) pero no en Loreto, representando especies con potencial ocurrencia en este departamento. Algunas de ellas son Lichonycteris degener, Sturnira erythromos o Vampyressa melissa, que por ahora no incluimos en la lista de especies.

De las siete especies "esperadas" para Loreto según Pitman et al. (2013), consideramos que solamente tres podrían ser encontradas en Loreto, principalmente por estar registradas en zonas adyacentes al departamento: Eumops bonariensis (Peters, 1874) y Molossus currentium Thomas, 1901, ambas presentes en el estado de Amazonas, Brasil (Dos Reis et al. 2007, Nogueira et al. 2014) y a Molossus pretiosus Miller, 1902 reportada en el departamento de Putumayo, Colombia (Ramírez-Cháves et al. 2013) y el estado de Amazonas, Brasil (Dos Reis et al. 2007, Nogueira et al. 2014).

Además, en nuestra lista incluimos 14 especies para Loreto no consideradas en la lista de Pitman et al. (2013): Anoura aequatoris (Patterson \& López Wong 2014), Hsunycteris pattoni (MUSM 31928), Cynomops milleri, Lampronycteris brachyotis y Phyllostomus latifolius (Pacheco et al. 2009), Carollia sp. sensu Solari \& Baker (2006) (Ruelas \& López 2018), Platyrrhinus fusciventris (Velazco et al. 2021), Peropteryx pallidoptera (Lim et al. 2010), Eumops maurus, E. delticus (Díaz 2011), Molossus alvarezi (González-Ruiz et al. 2011), Artibeus bogotensis (Calderón \& Pacheco 2012), Thyroptera wynneae (Velazco et al. 2014) y Hsunycteris dashe (Velazco et al. 2017).

Por otro lado, Fernández-Arellano y Torres-Vásquez (2013) incluyen en su recopilación de murciélagos de la Amazonia a Artibeus watsoni y Artibeus phaeotis para Loreto, basándose en las tesis de Saavedra (2010) y Cevillano y Ramos (2011) respectivamente. Sin embargo, la distribución confirmada de Artibeus watsoni va desde el Sur de México hasta el sureste de Panamá (IUCN) mientras que $A$. phaeotis se encuentra restringida a México y Centroamérica, siendo los especímenes de América del Sur, actualmente, Artibeus ravus (Hoofer et al. 2008). A. ravus está reportada en Perú, pero no en Loreto (Pacheco et al. 2009), quedando pendiente la revisión de esta especie. Lamentablemente, en las tesis mencionadas no se presentan los vouchers para estos especímenes, limitando su verificación.

Así mismo, formalmente no se encontraron localidades muestreadas en el Bosque Muy Húmedo Montano (BMHM). Aunque es la ecorregión de Loreto con menor área, podría contener especies diferentes porque forma parte de las Yungas (su altitud oscila entre los $1500 \mathrm{y}$ $2500 \mathrm{~m}$ ), conocidas por su alta diversidad de especies y endemismos (Pacheco et al. 2009, 2021). Esta ecorregión podría albergar especies montanas sugeridas por Pitman et al. (2013) para Loreto como 1) Eptesicus andinus, encontrada en la localidad de Cajaruru a $965 \mathrm{~m}$ de altitud en el departamento de Amazonas y en el Parque Nacional Río Abiseo (PNRA) a $2250 \mathrm{~m}$ de altitud en el departamento de San Martín (FMNH 106756, Davis \& Gardner 2008) y 2) Platyrrhinus ismaeli, encontrada en Amazonas a 1206 m y 1945 m de altitud y en San Martín, PNRA, a $2100 \mathrm{~m}$ y a $2211 \mathrm{~m}$ de altitud (Velazco \& Gardner 2009, Rengifo et al. 2011).

De las 78 especies registradas en alguna de las ANP de Loreto, 2 fueron reportadas sólo en el PNSD: Cyttarops alecto y Lonchorhina aurita (Ludeña \& Medina 2017, MUSM 17981) y una en el PNCAZ: Sturnira oporaphilum (Pacheco \& Arias 2001), mientras que 35 especies no se han registrado dentro de alguna ANP.

Endemismo, Conservación y Rareza de especies. - La reciente descripción de la especie endémica Hsunycteris dashe (Velazco et al. 2017) y el reconocimiento de Tonatia maresi y Glossophaga bakeri como especies válidas (Basantes et al. 2020, Velazco et al. 2021) hacen necesaria la evaluación de su categoría de Amenaza según las normas de la IUCN.

De la única especie en la categoría de Casi Amenazada, Vampyrum spectrum (IUCN), se tiene 10 registros en total para Loreto (Anexo 2, Tabla 1). Aunque por la cantidad de localidades (5, Tabla 1) en la que fue encontrada no la consideramos como una especie rara, el número de registros para la gran extensión del departamento de Loreto es bajo, por lo que se sugiere una reevaluación de los criterios de categorización para $V$. spectrum, ya que actualmente podría satisfacer los criterios para ser Vulnerable (IUCN, 2012). 
Con un mayor esfuerzo de muestreo e investigación enfocado en estas especies se podría tener mayor conocimiento de su historia natural y poblacional para categorizar a las especies apropiadamente, a la par que ocurren los importantes cambios taxonómicos, con el fin de tomar las medidas adecuadas para su conservación (Schipper et al. 2008).

\section{Vacíos de información. -}

1) Norte y centro de Putumayo. La provincia de $\mathrm{Pu}-$ tumayo, recientemente separada de Maynas en el 2014 (Ley $\mathrm{N}^{\circ}$ 30186) solo cuenta con 45 registros de murciélagos en los distritos de Yaguas y Putumayo (Montenegro \& Escobedo 2004, Bravo \& Borman 2008, Bravo 2010, Montenegro \& Moya 2011). Este bajo número puede explicarse por el difícil acceso a la zona y la gran extensión de territorio que está protegida bajo alguna ANP (P.N. Güeppí-Sekime, P.N. Yaguas, R.C Airo Pai, R.C. Humeiki, ACR Maijuna-Kichwa y ACR AmpiyacuApayacu). Tal como se documentó la diversidad biológica en el límite con Brasil (Escobedo 2003), sugerimos darle el énfasis al interfluvio Napo y Putumayo y a lo largo del río Putumayo porque soporta gran parte de la frontera con Colombia lo que podría permitir el registro de especies reportadas en zonas colombianas, pero no en Loreto. Es necesario también, completar inventarios que no cubrieron el grupo de murciélagos (Bravo \& Borman 2008, López Wong 2013, Bravo et al. 2016). De esta manera, se tendría un mejor conocimiento del número de especies para Loreto y por tanto para Perú (Schipper et al. 2008).

2) Noroeste de Maynas y noreste de Loreto y 3) Centro de Maynas. De los más de 8 mil registros de murciélagos de la provincia de Maynas, el 70\% se concentra en la carretera Iquitos-Nauta, en los distritos de San Juan Bautista e Iquitos y el 80\% de registros son contemporáneos (Hice et al. 2004, Montenegro \& Escobedo 2004, Quintana \& Pacheco 2007, Bravo \& Ríos 2007, Díaz 2011, Díaz \& Linares García 2012, Ruelas \& Pacheco 2015, Ruelas \& López 2018, Vásquez Újina 2019). Mientras que los distritos del centro y norte de Maynas (Torres Causana, Napo, Mazán y Alto Nanay), si bien presentan pocos registros, están restringidos a una zona en especial o son registros históricos (siglo XX) (Bravo \& Ríos 2007, Bravo \& Borman 2008, Bravo 2010, Ruelas y López 2018). Parte del $2^{\circ}$ 'vacío de información' cae en la cuenca de los ríos Napo y Curaray en los distritos de Napo y Torres Causana, zona que se solapa con el sitio prioritario para la conservación de la diversidad biológica de Loreto denominado 'Napo' (Mercado et al. 2016) donde se ha registrado una alta diversidad de murciélagos, resaltando la importancia de proteger las cabeceras de los ríos como el Arabela (Bravo \& Ríos 2007) pero que aún no se ha logrado oficialmente.
4) Centro-norte del Datem del Marañón, norte de Alto Amazonas y extremo occidental de Loreto. Esta zona abarca los distritos de Barranca, Pastaza y Andoas en el Datem del Marañón, Lagunas en Alto Amazonas y Urarinas en Loreto, sumando en total 221 registros en 22 localidades y 40 especies de murciélagos, entre los cuales están los tres hematófagos Desmodus rotundus, Diphylla ecaudata y Diaemus youngi, cuya presencia, sobretodo de D. rotundus, se debe a la existencia de zonas ganaderas (Bravo 2010). Esta zona no se encuentra protegida por ninguna ANP, sin embargo, se solapa con el sitio prioritario más grande para la conservación de la diversidad biológica de Loreto denominado 'Abanico del Pastaza' (Mercado et al. 2016) reconocido oficialmente por el Gobierno Regional de Loreto (GOREL) mediante la Ordenanza Regional N025-2016-GRL-CR, abarcando el territorio entre las laderas del río Pastaza y los ríos Marañón y Tigre.

5) Centro-sur de Requena y extremo oriental de Ucayali. Estas zonas están comprendidas en el corredor Ucayali-Yavarí, altamente diverso por la variedad de microhábitats que posee, sobre todo en el PNSD, ANP compartida con el departamento de Ucayali y limítrofe con Brasil. En la porción del PNSD correspondiente a Loreto se reportaron 52 especies de murciélagos (Jorge \& Velazco 2006, Medina et al. 2015), siendo la ANP más diversa del departamento; y a pesar de esto, se conoce muy poco acerca de las comunidades de murciélagos en la región. Este corredor comprende también el interfluvio Tapiche-Blanco, donde registraron 12 de las más de 100 especies de murciélagos esperadas para la zona (Escobedo 2015) y aunque aún no está protegida por el Estado, es parte del sitio prioritario para la conservación de la diversidad biológica de Loreto denominado 'Tapiche Blanco' (Mercado et al. 2016, O.R. Nº25-2016GRL-CR), lo cual es importante para completar el plan de conservación de la biodiversidad en todo el corredor Ucayali-Yavarí. Casi la mitad de los registros de Requena y Ucayali están concentrados en los distritos de Yaquerana (Requena) y Vargas Guerra (Ucayali); y a pesar que el $90 \%$ de estos registros son históricos, se determinaron dos especies nuevas y endémicas así como nuevos registros para Loreto a partir del estudio taxonómico de los especímenes colectados en esta zona (Simmons et al. 2002, Velazco et al. 2017, 2021), comprobando la importancia de colectar especímenes en campo que sirvan como vouchers de la diversidad de un lugar.

6) Extremo occidental de Ucayali. Esta zona de 'vacío de información' bañado por los ríos Pauya, Cushabatay, Pisqui y Tahuaya, que contribuyen al Bajo río Ucayali en Loreto, cubre los distritos de Pampa Hermosa y Contamana en la provincia de Ucayali, que en total cuentan con 139 registros repartidos en 13 localidades principalmente en el PNCAZ. 
Gracias a la exploración de las partes altas del Pauya y Pisqui en el 2000 (Pacheco \& Arias 2001), se encontraron 13 especies de murciélagos incluido Sturnira oporaphilum, que viene a ser el único registro de la especie para Loreto. No existen estudios de diversidad de murciélagos en el PNCAZ después de esta expedición y el resto de esta zona al oeste del río Ucayali permanece sin registros, resaltando una vez más la necesidad de explorar este extremo de Ucayali, sobretodo porque cuenta con importantes parches de Bosque Muy Húmedo Montano (BMHM).

Este trabajo está sustentado básicamente en el registro de especímenes de colección, pero novedosos métodos basados en las grabaciones acústicas de los murciélagos son cada vez más empleados en países vecinos como Brasil, Chile y Ecuador, que han logrado guías o claves de identificación acústica (Ossa et al. 2010, Rodríguez-San Pedro et al. 2016, Arias-Aguilar et al. 2018) o cuentan con una biblioteca virtual de las llamadas de ecolocación de murciélagos (Rivera et al. 2019). En Perú se ha seguido esta metodología acústica para detectar murciélagos en ecosistemas de la costa (Malo de Molina et al. 2011, Pacheco et al. 2015, Pacheco et al. 2020, Ugarte-Núñez 2020, Zegarra et al. 2020); pero lamentablemente ha sido poco aplicado en la selva. Sin duda, el mejor desarrollo de bibliotecas acústicas permitirá en un futuro cercano tener identificaciones más confiables y así completar las zonas de vacío de información en el departamento de Loreto y otras áreas del Perú, reforzar estudios previos y confirmar la presencia de especies potenciales o esperadas. Sin embargo, esto no debe ser considerado como reemplazo de las técnicas tradicionales como el uso de redes de neblina, búsqueda de refugios y colecta de vouchers sino como un método complementario del muestreo de tal manera que se pueda realizar estudios posteriores de taxonomía, ecología e incluso investigaciones sobre la relación hospedero-patógeno de enfermedades infecciosas emergentes como el reciente SARS-CoV-2 (Thompson et al. 2021).

El hecho de que la mayoría de nuestros registros contemporáneos (2001-2018) estén concentrados en Maynas, hace evidente la necesidad de fortalecer las instituciones como el Instituto de Investigaciones de la Amazonia Peruana (IIAP) y la Universidad Nacional de la Amazonia Peruana (UNAP) para que puedan liderar el desarrollo de inventarios biológicos en toda la extensión de Loreto. Se necesita también la capacitación de biólogos y conservacionistas locales, así como el apoyo del GOREL, la Autoridad Regional Ambiental (ARA) y otras instituciones como Derecho, Ambiente y Recursos Naturales (DAR) y Centro para el Desarrollo del Indígena Amazónico (CEDIA), para que promuevan la realización de estudios con el fin de conservar zonas con alta diversidad, no solo biológica, que es nuestro objetivo de estudio, sino cultural también. Recomendamos, al igual que Pitman et al. (2013), realizar inventarios en las zonas lotizadas para concesiones forestales, de hidrocarburos, minería y palma aceitera, a fin de colaborar con las evaluaciones de impacto ambiental (EIA) y sus planes de manejo, así como se realizó dentro del Lote 179 en los bosques entre la R.N Matsés y el P.N. Pacaya Samiria en Requena (Linares-Palomino et al. 2013).

Conociendo los vacíos de información, entendemos que existe una diversidad de murciélagos aún inexplorada cuyo papel ecológico en los ecosistemas naturales apenas empezamos a comprender y que está propensa a desaparecer por diversas amenazas (Schipper et al. 2008). Entre las más serias que afectan a los murciélagos se encuentran: la tala de árboles para la actividad maderera o para la agricultura intensiva, la creación de ciudades que trae consigo iluminación excesiva, ruido y contaminación química, la construcción de carreteras que crean barreras y la minería ilegal que trae contaminación. Estas amenazas conllevan a la fragmentación de hábitats que desencadena una menor tasa reproductiva (Voigt \& Kingston 2016, Altringham \& Kerth 2016). La conservación de las especies de murciélagos va a requerir de estudios a largo plazo para evaluar el estado y tendencias de la población y así tener respuestas clave para la conservación de los murciélagos tropicales (Meyer et al. 2016, Frick et al. 2019).

Un gran avance es que el gobierno regional de Loreto aunado a otras instituciones está disponiendo sitios ecológicamente valiosos, como las cuencas del canal principal del Marañón y del río Morona (Varillales Bajo Morona), del río Huallaga (Kampu Piyawi) y del río Yavarí (Yavarí Mirim), entre otros mencionados anteriormente, promoviendo a la vez el desarrollo local y regional a través del aprovechamiento de recursos de manera sostenible y regulada, mediante el establecimiento de estos sitios prioritarios para la conservación de la diversidad biológica de Loreto (Mercado et al. 2016) promulgados por la Ordenanza Regional $N^{\circ} 025$ 2016-GRL-CR.

\section{Conclusiones}

El departamento de Loreto es uno de los que mayor diversidad de murciélagos tiene en el Perú, con 114 de las 189 especies; sin embargo, los más de 10 mil registros aquí recopilados están en su mayoría concentrados en solo 3 de las 8 provincias que lo constituyen, reflejando 6 zonas de vacíos de información importantes. Se resalta que una gran parte de su territorio está a la espera de exploraciones que nos acerquen a la verdadera diversidad que alberga Loreto, lo que se logrará realizando inventarios que combinen métodos novedosos como bioacústica y colecta de especímenes a través de métodos tradicionales como el uso de redes de neblina. 


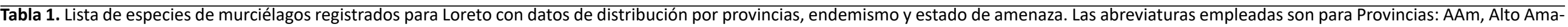

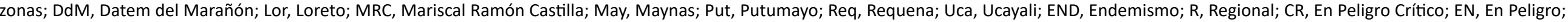

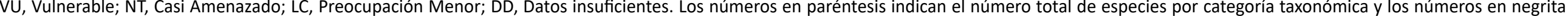

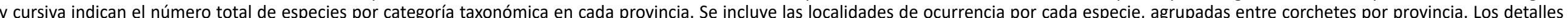

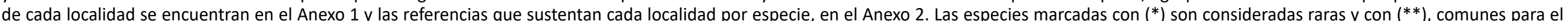
departamento de Loreto.

\section{Nombre cientifico}

Nombre común

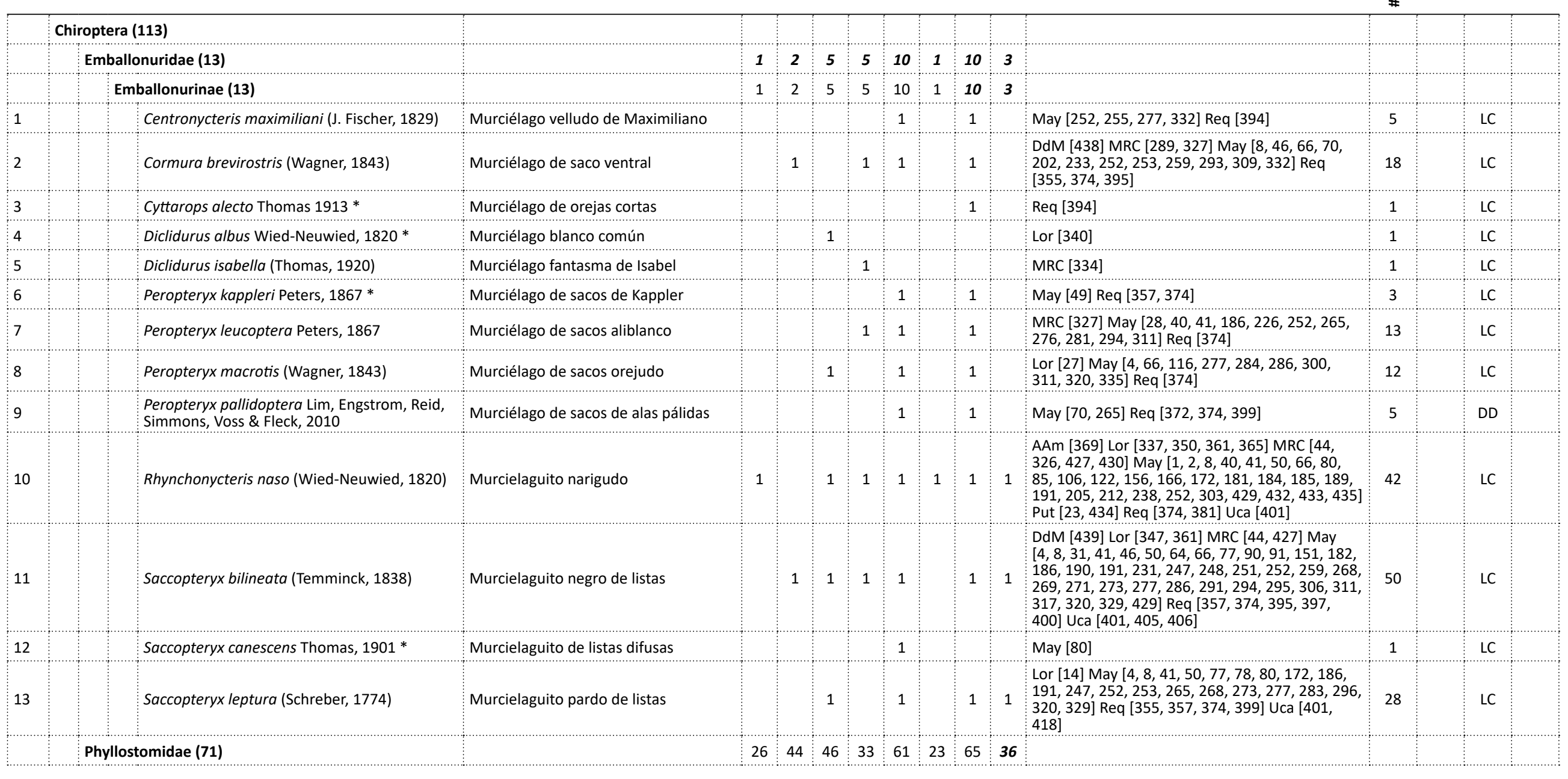




\begin{tabular}{|c|c|c|c|c|c|c|c|c|c|c|c|c|c|c|}
\hline & Desmodontinae (3) & & 1 & 3 & 2 & 1 & 3 & 0 & 2 & 1 & & & & \\
\hline 14 & Desmodus rotundus (E. Geoffroy, 1810) & Vampiro común & 1 & 1 & 1 & & 1 & & 1 & & $\begin{array}{l}\text { AAm [388] DdM [298, 301,343, 360, 371] Lor } \\
{[14,22,325,338,345,348] \text { May }[1,3,8,40,41} \\
53,54,57,77,169,178,182,183,186,188,210, \\
225,231,232,244,247,252,261,277,294,296, \\
300,310-312,316,320,323] \text { Req }[355,357,374]\end{array}$ & 53 & & LC \\
\hline 15 & Diaemus youngi (Jentink, 1893) & Vampiro aliblanco & & 1 & & 1 & 1 & & & & DdM $[298,301,360]$ MRC [47] May [57] & 5 & & LC \\
\hline \multirow[t]{2}{*}{16} & Diphylla ecaudata Spix, 1823 & Vampiro peludo & & 1 & 1 & & 1 & & 1 & 1 & $\begin{array}{l}\operatorname{DdM}[256,298,302,360,371] \text { Lor [14] May } \\
{[316,437] \text { Req }[374,397-399] \text { Uca [424] }}\end{array}$ & 13 & & LC \\
\hline & Glossophaginae (5) & & 1 & 4 & 4 & 1 & 4 & 2 & 3 & 1 & & & & \\
\hline 17 & Anoura aequatoris (Lönnberg, 1921) * & Murciélago longirostro de Ecuador & & 1 & & & & & & & $\operatorname{DdM}[383]$ & 1 & & $\mathrm{LC}$ \\
\hline 18 & Anoura caudifer (E. Geoffroy, 1818) & Murciélago longirostro menor & & 1 & 1 & & 1 & 1 & 1 & 1 & $\begin{array}{l}\operatorname{DdM}[353] \text { Lor }[10,11,13,14,96] \text { May }[3,4,5, \\
7,8,41,50,55,253,316] \text { Put }[30,431] \text { Req }[357, \\
397,398] \text { Uca }[402,405]\end{array}$ & 23 & & LC \\
\hline 19 & Choeroniscus minor (Peters, 1868) & Murcielaguito longirostro amazónico & & 1 & 1 & & 1 & & 1 & & $\begin{array}{l}\operatorname{DdM}[304,333,343,438] \text { Lor }[14,27,347,361] \\
\text { May }[1,51,74,218,252,272,277,286,294, \\
296,307,312,320,322,328] \text { Req }[355,357, \\
374,399]\end{array}$ & 27 & & LC \\
\hline 20 & Glossophaga bakeri Webster and Jones, 1987 & Murciélago longirostro de Baker & & & 1 & & 1 & & & & $\begin{array}{l}\text { Lor }[35,68,361] \text { May }[50,56,114,210,231 \text {, } \\
320]\end{array}$ & 9 & & LC \\
\hline \multirow[t]{2}{*}{21} & Glossophaga soricina (Pallas, 1766) ** & Murciélago longirostro de Pallas & 1 & 1 & 1 & 1 & 1 & 1 & 1 & & $\begin{array}{l}\text { AAm [391] DdM [17] Lor [10,14, 22, 168, 170, } \\
348,350,366,382] \text { MRC }[84,327] \text { May }[3,5,8, \\
28,50,54,55,62,70,76,81,83,87,89,90,94 \\
98,107,109,114,122,123,126,127,129,136, \\
142,143,150,151,153,157,158,159,160,162- \\
164,169,173,174,178-184,187-189,191,192, \\
195,197,199,202,205,209-213,220,223,224, \\
227,231,234-238,241,247,249,250,252,253, \\
255,260,265,266,268,270,271,273,275,276, \\
278,285,288,292-296,305,309,310,312-316, \\
318,320,324,329,335,435] \text { Put [434] Req [355, } \\
357,374,399,408,411]\end{array}$ & 131 & & LC \\
\hline & Lonchophyllinae (4) & & 0 & 2 & 2 & 0 & 3 & 0 & 4 & 1 & & & & \\
\hline 22 & $\begin{array}{l}\text { Hsunycteris dashe Velazco, Soto-Centeno, } \\
\text { Fleck, Voss and Simmons, } 2017^{*}\end{array}$ & Murciélago nectarívoro de Dashe & & & & & & & 1 & & Req [374] & 1 & $\mathrm{R}$ & \\
\hline 23 & Hsunycteris pattoni (Woodman y Timm, 2006) & Murciélago longirostro de Patton & & & & & 1 & & 1 & & May $[77,324]$ Req $[355,357,374]$ & 5 & & DD \\
\hline 24 & Hsunycteris thomasi (J. A. Allen, 1904) & Murciélago longirostro de Thomas & & 1 & 1 & & 1 & & 1 & 1 & $\begin{array}{l}\operatorname{DdM}[342,383,387] \text { Lor }[14,19,35,347,350, \\
361] \text { May }[1,39,49,50,75,81,90,166,173, \\
180-182,187,190,195,197,202,204,210,213 \\
217,233,234,236,242,244,247,248,251-253, \\
261,265,266,270,273,275,276,278,279,281, \\
292,294,295,315-317,320,324,328,329,436] \\
\text { Req [355, 357, 374, 394, 397] Uca [420] }\end{array}$ & 67 & & LC \\
\hline 25 & Lionycteris spurrelli Thomas, 1913 & Murciélago longirostro pequeño & & 1 & 1 & & 1 & & 1 & & $\begin{array}{l}\text { DdM }[341,343] \text { Lor }[338] \text { May }[4,59,260] \text { Req } \\
{[443]}\end{array}$ & 7 & & LC \\
\hline
\end{tabular}




\begin{tabular}{|c|c|c|c|c|c|c|c|c|c|c|c|c|c|c|c|}
\hline & Lonchorrhinae (1) & & 0 & 0 & 0 & 0 & 0 & 0 & 0 & 1 & & & & & \\
\hline \multirow[t]{2}{*}{26} & Lonchorhina aurita Tomes, $1863 *$ & Murciélago de espada & & & & & & & & 1 & Uca [405] & 1 & & LC & \\
\hline & Micronycterinae (7) & & 0 & 3 & 3 & 2 & 4 & 1 & 7 & 1 & & & & & \\
\hline 27 & Lampronycteris brachyotis (Dobson, 1879) * & Murciélago de garganta amarilla & & & & & 1 & & 1 & & May $[1,50] \operatorname{Req}[357,374]$ & 4 & & LC & \\
\hline 28 & $\begin{array}{l}\text { Micronycteris (Leuconycteris) brosseti Sim- } \\
\text { mons y Voss, 1998* }\end{array}$ & Murciélago orejudo de pliegues altos & & & 1 & 1 & 1 & & 1 & & Lor [69] MRC [327] May [66] Req [357] & 4 & & LC & \\
\hline 29 & $\begin{array}{l}\text { Micronycteris (Xenoctenes) hirsuta (Peters, } \\
1869)^{*}\end{array}$ & Murciélago de orejas peludas & & 1 & & & & & 1 & & DdM [304, 336] Req [374] & 3 & & LC & \\
\hline 30 & $\begin{array}{l}\text { Micronycteris (Micronycteris) matses Sim- } \\
\text { mons, Voss, y Fleck, } 2002^{*}\end{array}$ & Murciélago orejudo matsés & & & & & & & 1 & & $\operatorname{Req}[374,397]$ & 2 & $\mathrm{R}$ & DD & DD \\
\hline 31 & $\begin{array}{l}\text { Micronycteris (Micronycteris) megalotis (Gray, } \\
1842 \text { ) }\end{array}$ & Murciélago orejudo pequeño & & 1 & 1 & 1 & 1 & 1 & 1 & 1 & $\begin{array}{l}\text { DdM }[67,383,387] \text { Lor }[19,96] \text { MRC }[84,327] \\
\text { May }[40,41,59,66,182,195,199,252,253, \\
260,264,295,300,305,308,317,320,323,328, \\
329] \text { Put }[15] \text { Req }[355,357,374] \text { Uca }[402,405]\end{array}$ & 33 & & LC & \\
\hline 32 & $\begin{array}{l}\text { Micronycteris (Micronycteris) microtis Miller, } \\
1898^{*}\end{array}$ & Murcielago orejudo común & & & 1 & & & & 1 & & Lor [29] Req [374] & 2 & & LC & \\
\hline \multirow[t]{2}{*}{33} & $\begin{array}{l}\text { Micronycteris (Schizonycteris) minuta (Ger- } \\
\text { vais, 1856) }\end{array}$ & Murciélago orejudo de pliegues altos & & 1 & & & 1 & & 1 & & $\begin{array}{l}\text { DdM [33] May [4, 8, 139, 186, 223, 231, 248, } \\
252,258,268,311,320] \text { Req }[357,374]\end{array}$ & 15 & & LC & \\
\hline & Phyllostominae (17) & & 6 & 6 & 8 & 6 & 16 & 6 & 16 & 6 & & & & & \\
\hline 34 & Chrotopterus auritus (Peters, 1856) & Falso vampiro & & & & & 1 & & 1 & & $\begin{array}{l}\text { May }[1,40,41,182,186,190,218,251,252, \\
268,277,286,311] \text { Req }[354,357,374,442]\end{array}$ & 17 & & LC & \\
\hline 35 & Gardnerycteris crenulatum (E. Geoffroy, 1803) & Murciélago de hoja nasal peluda & 1 & 1 & 1 & 1 & 1 & & 1 & 1 & $\begin{array}{l}\text { AAm [386] DdM [341] Lor [14] MRC [331] May } \\
{[1,8,31,34,40,41,50,62,76,77,81,90,150,} \\
169,171,173,182,190,191,195,199,200,202, \\
204,210,213,220,226,227,232,233,236,252, \\
253,259,260,264-266,270,276,281,285,287, \\
290-295,305,308,309,312,314-316,320,329, \\
437] \text { Req [355, 357, 374] Uca [405] }\end{array}$ & 68 & & LC & \\
\hline 36 & Glyphonycteris daviesi (Hill, 1964) & Murciélago orejudo de Davies & & & & & 1 & 1 & 1 & & $\begin{array}{l}\text { May }[65,71,186,277,282,283,300] \text { Put }[434] \\
\text { Req }[374]\end{array}$ & 9 & & LC & \\
\hline 37 & Glyphonycteris sylvestris Thomas, 1896 & Murciélago de pelaje tricoloreado & & & & & 1 & & 1 & & $\begin{array}{l}\text { May [244, 259, 268, 272, 286, 288, 293, 309, } \\
\text { 311] Req [374] }\end{array}$ & 10 & & LC & \\
\hline 38 & Lophostoma brasiliense Peters, 1866 & Murciélago de orejas redondas pigmeo & & & & & 1 & & 1 & & $\begin{array}{l}\text { May }[1,8,232,247,252,320] \text { Req }[355,357 \text {, } \\
374,399]\end{array}$ & 10 & & LC & \\
\hline 39 & Lophostoma carrikeri (J. A. Allen, 1910) & Murciélago orejudo de vientre blanco & & & & 1 & 1 & 1 & 1 & & $\begin{array}{l}\text { MRC [427] May [186, 231, 287, 320] Put [36] } \\
\text { Req [357] }\end{array}$ & 7 & & LC & \\
\hline
\end{tabular}




\section{Nombre científico}

Nombre común

\section{较}

DdM $[67,72,298,304,333,342,343]$ Lor $[14$, 347, 366] MRC [84, 426, 428] May [1, 3, 4, 8, 31, $34,39,41,50,66,70,71,77,82,90,106,180$, $187,190,199,202,204,209,210,233,236,249$ $250,251,252,255,261,265,268,274,278,281$, $283,291,293,295,305,306,312,315,316,318$ $320,329,330,335,429,432,435-437]$ Put [38] (401, $402,412,415]$

Murciélago de orejas redondas de garganta blanca

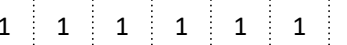

Macrophyllum macrophyllum (Schinz, 1821) * Murciélago pernilargo

Phylloderma stenops Peters, 1865

Murciélago de rostro pálido
Trachops cirrhosus (Spix, 1823)

\section{$\begin{array}{l:l:l}1 & 1 & \text { Req [374] Uca [405] }\end{array}$}

Lor [14, 359] May $[4,55,59,186,222,232,252$, $269,313,318,322,332]$ Req $[354,355,357]$ AAm [391] Lor [368] MRC [427, 428] May [54, $57,62,81,87,98,100,105,122,126,127,129$, $135,149,150-153,155,158,174,179,183,196$ $197,210,225,231,235,242,252,267,292,296$

AAm [388] DdM [16, 37, 438] Lor [11, 14, 29, $347,350,368]$ MRC $[327,426,427,429]$ May $[1$, $3,4,8,39,40,41,50,55,56,58,66,77,87,106$ $151,178,182,186,190,218,231,248,252,261$ $265,268,269,276,280,283,291,293,295,309$ 30, 431, 329 $3354,355,353,374,379] 384,392$, $397,408,440]$ Uca $[401,405,424]$

AAm [391] DdM [37, 45, 67, 298] Lor [14, 347, 361, 382] May [8, 41, 50, 58, 59, 62, 66, 70, 76, 81, $1704,106,139,148,151,158,160,169$,

$210,214,218,220,226,229,230,231,233,235$

$237,242,247,249,250-252,255,257,258,262$

$265,270,271,273,276,281,282,285,287,288$ $291-293,295,296,309,312,314,316,318,320$ $322,329,429,433]$ Put [38] Req [355-357, 374, $394,395,398,399,440]$ Uca [401, 406]

\section{May [4]}

AAm [387] Lor [350] May [1, 40, 41, 50, 59, 188 $218,226,227,247,251,252,261,265,269,270$ $273,274,280,283,285,287,291,292,295,296$ $309,312,316,318,320,323,328,332,437]$ Req $[357,374,392,442]$

DdM $[256,360]$ Lor $[14,338,347,350,359]$ MRC [47, 327, 426, 427] May [1, 3, 31, 39-41, 50, 55, 


\begin{tabular}{|c|c|c|c|c|c|c|c|c|c|c|c|c|c|c|c|}
\hline & Nombre científico & Nombre común & $\frac{\xi}{\alpha}$ & $\sum_{\mathrm{O}}$ & ذَ & 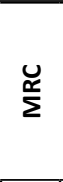 & $\sum^{\mathrm{\pi}}$ & $\stackrel{\Xi}{a}$ & $\underset{\mathscr{\varpi}}{\mathscr{\varpi}}$ & Ј & Localidades & 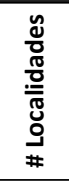 & $\sum_{w}^{0}$ & ż & 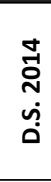 \\
\hline 49 & Trinycteris nicefori (Sanborn, 1949) & Murciélago de orejas puntiagudas & 1 & 1 & & & 1 & & 1 & & $\begin{array}{l}\text { AAm [388] DdM [342] May [41, 50, 66, } 171,181 \text {, } \\
209,222,248,251,252,258,269,280,293,320 \text {, } \\
323,329,330] \text { Req }[357,374]\end{array}$ & 22 & & LC & \\
\hline \multirow[t]{2}{*}{50} & Vampyrum spectrum (Linnaeus, 1758) & Gran falso vampiro & & & & & 1 & & 1 & & May $[244,251,263,300]$ Req $[357]$ & 5 & & NT & \\
\hline & Carolliinae (6) & & 5 & 6 & 6 & 5 & 6 & 4 & 6 & 4 & & & & & \\
\hline 51 & Carollia benkeithi Solari y Baker, 2006 & Murciélago frutero de Ben Keith & 1 & 1 & 1 & 1 & 1 & & 1 & 1 & $\begin{array}{l}\text { AAm [388] DdM [342, 360] Lor [347, 350, 361, } \\
365,368,382] \text { MRC }[426,428] \text { May }[329] \text { Req } \\
{[355,357,374,397,399] \text { Uca [402, 405, 424] }}\end{array}$ & 20 & & LC & \\
\hline 52 & Carollia brevicauda (Schinz, 1821) ** & Murciélago frutero colicorto & 1 & 1 & 1 & 1 & 1 & 1 & 1 & 1 & $\begin{array}{l}\text { AAm [388, 391] DdM }[16,72,256,304,319, \\
341-343,358,364,370,377,378,438,439] \text { MRC } \\
{[430] \text { Lor }[11,12,14,24,68,208,337,338,344,} \\
347,350,361,365] \text { May }[1,34,4,8,28,41,49, \\
50,55,58,60,65,76,77,79,80,81,83,85,90, \\
114,139,140,143,146,153,158,160,161,167, \\
169,171-173,175,178-182,184,187-189,191, \\
192,195-197,199,201,202,204,205,207,209, \\
210,212-214,217,218,220,221,223,226,230- \\
234,236,238,241-244,247,249,250-253,255, \\
259-261,264-266,269,270,273,274,276-288, \\
290,292-296,300,308-310,312-318,320,323, \\
328,329,332,335,436,437] \text { Put }[15] \text { Req [355, } \\
357,374,394,395,397-399,407,440-442] \text { Uca } \\
{[396,405,412,413,415-417,419,420]}\end{array}$ & 185 & & LC & \\
\hline 53 & Carollia perspicillata (Linnaeus, 1758) ** & Murciélago frutero común & 1 & 1 & 1 & 1 & 1 & 1 & 1 & 1 & $\begin{array}{l}\text { AAm [388, 391] DdM }[16,17,25,37,45,67,72, \\
298,336,341,342,343,352,360,371,375] \text { Lor } \\
{[10-14,19,20,22,24,27,68,69,168,208,339,} \\
344,347,350,359,361,365,438] \text { MRC }[327, \\
426-428,430] \text { May }[1,3,4,7,8,28,32,41,50, \\
52-58,60,64,66,70,74,76,77,79,80,81,83, \\
85-87,90,93,98,106,110-122,129,134,139, \\
140,142-144,146,151-153,157,159,160,163, \\
164,166,169,171-173,175,177-184,186-192, \\
195,196,199,200,202,204,205,207,209, \\
210-215,217,218,220,223-225,228-239,241, \\
244,245,247-253,255,257,260,261,263-267, \\
270-274,276,277,279,280-288,290-296,300, \\
303,306,309,310,312-316,318,320,323,328, \\
329,335,429,433,436,437] \text { Put }[6,15,23,30, \\
431] \text { Req }[355,357,374,379,398-400,407,440, \\
442] \text { Uca }[396,401,405,412,413,416,417, \\
419,420,423]\end{array}$ & 240 & & LC & \\
\hline
\end{tabular}


Nombre científico

Nombre común

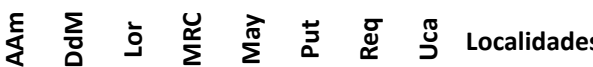

$\operatorname{DdM}[37,256,343]$ Lor $[10,11,13,14,22,24$, 29, 48, 338] MRC [430] May [3, 4, 5, 8, 41, 50,

$51,55,62,66,7476,79,81,90,173,178,179$

$180,181,182,188,191,195,197,199,201,202$

$204,209,210,213,217,220,230,233,234,236$,

$241,247,248,252,253,260,265,267,270,272$ $273,276,277,279,280-282,285-288,292-296$, $335,429]$ Put $[15,30,38,431]$ Req $[357,374]$

AAm [388] DdM [256] Lor [10, 14, 19, 21, 22, 347] May [1, 3, 39-41, 55, 58, 59, 76, 77, 199, $202,206,212,218,230,236,252,253,260,261$, $270,273,274,278,281,287,288,253,260,261$, $374,394,398,399]$

AAm [388] DdM [16, 17, 37, 67, 73, 304, 342] Lor $[11,13,14,19,22,29,345,347,361,368]$ MRC $[430]$ May $[1,3,4,5,8,28,31,40,41,49$, $50,52,55,58-60,63,66,74,76,77,81,83,90$, $191,195,197,199,202,204,209,210,212,213$

218, 220, 221, 223, 233-236, 239, 241-243, $245-253,260,261,263,265,266,270,273,274$ $276,277,279-281,283,285-288,292-296,308-$ $310,312-318,320,322-324,328,329,335,436$ 437] Put $[23,38]$ Req $[355,357,374,394,395$, $397,399,407,440-442]$ Uca [420]

\section{Stenodermatinae (28)}

Artibeus anderseni Osgood, 1916

Artibeus bogotensis Andersen, 1906 *
Murciélago frugívoro de Bogotá

AAm $[388,391] \operatorname{DdM}[25,45,73,256,304,341$, $343,360]$ Lor [11, 325, 338, 344, 361, 368] MRC $[84,327,428]$ May $[1,8,41,46,50,51,53,57$ $62,63,64,66,75,77,80,81,83,87,90,139$,

$142,144,147,157,158,160,171,173,178-181$

$184,188,189,196-198,202,206,209-211,220$, $221,223,231,233-235,237,240,243,252,255$ $266,270-272,276,280,285,288,292,293,255$, $357,367,374,379]$ ]

Req [357]

May [252, 329] Req [395, 397-399]

DdM [25 May [127, 142, 160, 183, 212, 214, 232

$244,252,259,266,322]$ Req [357]

Murcielaguito frugívoro pardo

1

1

$\begin{array}{llllll:l:l:l:l}13 & 20 & 21 & 18 & 25 & 10 & 27 & 21\end{array}$

DdM [341] Lor [208, 350] May [80,182,316,

Murciélago frutero plateado 


\begin{tabular}{|c|c|c|c|c|c|c|c|c|c|c|c|c|c|c|c|}
\hline & Nombre científico & Nombre común & $\frac{\varepsilon}{\alpha}$ & $\sum_{0}$ & ذَ & 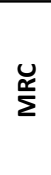 & $\sum^{\frac{\pi}{2}}$ & $\stackrel{ \pm}{a}$ & ष্̋ & ঠூ & Localidades & 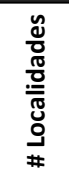 & $\sum_{u}$ & ż & 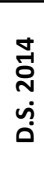 \\
\hline 62 & Artibeus gnomus Handley, 1987 & Murciélago frutero enano & 1 & 1 & 1 & & 1 & & 1 & & $\begin{array}{l}\text { AAm [388] DdM [17, 37, 304, 341, 343] Lor [13, } \\
14,48,68,92,347] \text { May }[1,40,52,59,71,173, \\
177,179,182,187,188,190,195,204,207,210- \\
213,218,226,230,232,235,236,242,247,250, \\
252,253,255,265,266,269,278,279,281-283, \\
285,287,288,293,295,305,310,312,316,318, \\
320,328,329,332] \text { Req }[354,357,374,394, \\
395,397-399]\end{array}$ & 73 & & LC & \\
\hline 63 & Artibeus lituratus (Olfers, 1818) ** & Murcielaguito frugívoro mayor & 1 & 1 & 1 & 1 & 1 & 1 & 1 & 1 & $\begin{array}{l}\text { AAm [387, 388, 391] DdM [37, 45, 67, 72, 73, } \\
299,301,302,304,341,360,371,383,439] \text { Lor } \\
{[11,18,22,325,337,347,382] \text { MRC }[44] \text { May }} \\
{[1,3,4,41,46,50,55,56,63,65,75-77,79,81,} \\
83,90,93,98106,110,111,114,125-127,131, \\
139,142,145,146,150,151,158-160,162,164, \\
166,169,171,172,180-183,188,189,191,195, \\
196,198,199,202,204,209-213,218,221,226, \\
231,233,234,236-238,240,241,243,244,247, \\
249-253,255,257,260,265,266,270,273,276, \\
279,280-282,285,286,288,292-294,305,309, \\
312,314,316-318,320,329,335,429] \text { Put }[23] \\
\text { Req [355, 357, 374, 395, 398, 399, 407, 440, } \\
441] \text { Uca [405, 414] }\end{array}$ & 145 & & LC & \\
\hline 64 & Artibeus obscurus (Schinz, 1821) ** & Murcielaguito frugívoro negro & 1 & 1 & 1 & 1 & 1 & 1 & 1 & 1 & $\begin{array}{l}\text { AAm [376, 388] DdM [16, 37, 67, 73, 256, 298, } \\
302,319,343,439] \text { Lor }[14,19,22,29,325,337, \\
338,344,347,350,351,361,365] \text { MRC }[331, \\
426,427,430] \text { May }[1,3,4,28,32,41,46,50, \\
55,59,63,65,71,74,76,77,79,81,110,146, \\
164,172,180-182,187,190,195,197,199,202, \\
206,209,210,212-214,217,218,220,221,223, \\
225,231,233,234,236,240-242,247,251-253, \\
257,260,265,266,269,270,273,274,276,280- \\
283,285,287,288,290-296,309,310,312-314, \\
316-318,320,323,329,335,429,432,433,435, \\
436] \text { Put }[36,38] \text { Req }[355,357,374,379,394, \\
398,399,407,408,411,440-442] \text { Uca }[405,412, \\
420]\end{array}$ & 141 & & LC & \\
\hline
\end{tabular}


Nombre científico

Nombre común

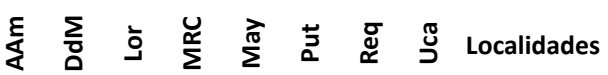

\begin{tabular}{|c|c|c|c|c|c|c|c|c|c|c|c|c|c|}
\hline 65 & Artibeus planirostris (Spix, 1823) ** & Murciélago frutero de rostro plano & 1 & 1 & 1 & 1 & 1 & 1 & 1 & 1 & $\begin{array}{l}\text { AAm [388, 391] DdM [67, 73, 256, 301, 304, 341, } \\
\text { 342, 360, 371, 439] Lor [11, } 14,21,168,208, \\
325,337,338,344,347,350,361,365,368,382] \\
\text { MRC }[44,84,327,426-428] \text { May }[1,3,4,5,8, \\
28,41,46,50,54,55,57,58,60,65,74,75,76, \\
80,81,83,90,93-95,100,102,104,106-108, \\
110,111,114,115,125-127,129,134,135,139, \\
142-144,146,147,149,151-153,155,158-164, \\
166,169,171-173,177-182,184,187-191,195- \\
199,202,204,209,210,212,213,217,220,221, \\
225-227,230,233-237,240-242,244,247,249, \\
250-253,260,265,266,269,270,272,273,275, \\
276,280,282,285,287,288,292-294,296,309, \\
312,314,315,318,320,329,332,335] \text { Put }[38, \\
431] \text { Req }[355,357,374,394,395,397-399,407, \\
410,411,440,441] \text { Uca } 405,415,420]\end{array}$ & 187 & LC \\
\hline 66 & Chiroderma salvini Dobson, 1878 & Murciélago de listas claras & & & & 1 & 1 & & 1 & & $\begin{array}{l}\text { MRC }[426,428] \text { May }[77,179,230,252,285, \\
\text { 295] Req [398] }\end{array}$ & 9 & LC \\
\hline 67 & Chiroderma trinitatum Goodwin, 1958 & Murciélago menor de listas & & 1 & 1 & 1 & 1 & & 1 & 1 & $\begin{array}{l}\text { DdM [256] Lor [22, 347, 368] MRC [327] May } \\
{[40,41,83,179,198,202,207,218,226,232,} \\
240,241,243,249,252,260,282,310-312] \text { Req } \\
{[357,395,398,399,441] \text { Uca [405] }}\end{array}$ & 31 & LC \\
\hline 68 & Chiroderma villosum Peters, 1860 & Murciélago de lineas tenues & & 1 & 1 & 1 & 1 & & 1 & 1 & $\begin{array}{l}\text { DdM [298, 342, 352] Lor [208, 325, 337, 347, } \\
361,365,382] \text { MRC [44, 327] May [1, 3, 41, 46, } \\
50,55,59,75,176,188,191,195,198,226,240, \\
252,255,258,270,280,285,288,293,309,312, \\
316,320] \text { Req [357, 395, 399] Uca [415, 424] }\end{array}$ & 44 & LC \\
\hline 69 & Enchisthenes hartii (Thomas, 1892) * & Murciélago frutero aterciopelado & & & & 1 & & & 1 & 1 & $\operatorname{MRC}[427,428]$ Req $[357,440]$ Uca $[425]$ & 5 & LC \\
\hline 70 & Mesophylla macconnelli Thomas, 1901 & Murcielaguito cremoso & 1 & 1 & 1 & 1 & 1 & 1 & 1 & 1 & $\begin{array}{l}\text { AAm [388] DdM [16, 45] Lor [13, } 14,22,92,208, \\
347,350,368] \text { MRC }[430] \text { May }[1,2,4,5,8,28, \\
32,49,55,57,59,63,70,75,173,176,179,181, \\
182,188,190,202,210,218,220,223,226,247, \\
250-253,259,260,263,279-282,287,288,292, \\
294-296,306,311,312,314-318,320,323,329, \\
332,433] \text { Put }[434] \text { Req }[355,357,367,374,394, \\
395,398,399,408,411,440] \text { Uca }[401,405, \\
412,420]\end{array}$ & 86 & LC \\
\hline 71 & $\begin{array}{l}\text { Platyrrhinus brachycephalus (Rouk y Carter, } \\
\text { 1972) }\end{array}$ & $\begin{array}{l}\text { Murciélago de nariz ancha de cabeza } \\
\text { pequeña }\end{array}$ & 1 & 1 & 1 & 1 & 1 & & 1 & 1 & $\begin{array}{l}\text { AAm [390, 391] DdM [26, 256, 298, 341, 343] } \\
\text { Lor [10-12, 14, 325, 337, 344, 345, 350, 361, } \\
365] \text { MRC [84, 327] May }[4,40,46,53,57,62, \\
76,77,90,123,129,130,135,139,146,149, \\
150,156,158-160,166,169,174,178,180,181 \\
183,196,202,206,209,221,222,231,234,235 \\
240,241,252,255,258,276,281,282,285,292 \\
\text { 295, 296, 310, 312, 316, 329] Req [355, 357, } \\
397-399,441] \text { Uca [420] }\end{array}$ & 80 & LC \\
\hline 72 & $\begin{array}{l}\text { Platyrrhinus fusciventris Velazco, Gardner y } \\
\text { Patterson, } 2010^{*}\end{array}$ & $\begin{array}{l}\text { Murciélago de nariz ancha y vientre } \\
\text { marrón }\end{array}$ & & & & & & & 1 & & Req $[354,355]$ & 2 & LC \\
\hline
\end{tabular}


Nombre científico

Nombre común

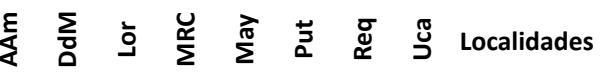

\begin{tabular}{|c|c|c|c|c|c|c|c|c|c|c|c|c|c|c|}
\hline 73 & Platyrrhinus incarum (Thomas, 1912) & Murciélago de nariz ancha inca & 1 & 1 & 1 & 1 & 1 & & 1 & & $\begin{array}{l}\text { AAm [388, 390] DdM [25, 342] Lor [10, } 14,325, \\
365,382] \text { MRC }[44,327] \text { May }[1,4,40,41,46, \\
50,64,70,83,90,130,164,176,180,183,184 \\
187,188,195,197,199,202,204,207,209,210, \\
212,213,221,226,233-235,237,240-242,246, \\
247,251-253,260,266,270,273,276,279,280, \\
282,285,287,288,291-293,295,307,309-312, \\
314,316,323,330,335,437] \text { Req }[354,355 \\
357,394,395,398,399,441]\end{array}$ & 87 & LC & \\
\hline 74 & Platyrrhinus infuscus (Peters, 1880) & $\begin{array}{l}\text { Murciélago de nariz ancha de listas } \\
\text { tenues }\end{array}$ & & 1 & 1 & & 1 & & 1 & 1 & $\begin{array}{l}\operatorname{DdM}[297,439] \text { Lor }[12,325] \text { May }[1,8,76,252, \\
310,322] \text { Req }[374,411,440] \text { Uca }[405]\end{array}$ & 14 & LC & \\
\hline 75 & Sphaeronycteris toxophyllum Peters, 1882 & Murciélago apache & & & 1 & 1 & 1 & & & 1 & $\begin{array}{l}\operatorname{Lor}[11,12,325] \text { MRC }[47] \text { May }[1,77,127,212] \\
\text { Uca }[401]\end{array}$ & 9 & LC & DD \\
\hline 76 & Sturnira giannae Velazco y Patterson, 2019 & $\begin{array}{l}\text { Murciélago de charreteras amarillas } \\
\text { de Gianna }\end{array}$ & 1 & 1 & 1 & 1 & 1 & 1 & 1 & 1 & $\begin{array}{l}\text { AAm [387, 391] DdM [9, 301, 371] Lor [10-12, } \\
14,68,208,337,338,345,350,365] \text { MRC [44, } \\
84] \text { May }[1,3,8,46,50,62,75,76,83,90,150, \\
151,158,169,173,178-181,183,187,188,191, \\
195,197,199,202,204,210-213,216,220,223, \\
231-236,239,241,242,244,247,249-253,255, \\
260,265-268,270,271,273,275,276,282,285, \\
287,288,292,293,295,296,305,309,314,315, \\
320,335,437] \text { Put [23] Req [355, 357, 397] Uca } \\
{[402,424]}\end{array}$ & 101 & & \\
\hline 77 & Sturnira magna de la Torre 1966 & $\begin{array}{l}\text { Murciélago de hombros amarillos } \\
\text { grande }\end{array}$ & 1 & 1 & 1 & 1 & 1 & 1 & 1 & 1 & $\begin{array}{l}\text { AAm [386] DdM [256, 387] Lor [14, 20, 21, 22, } \\
338] \text { MRC [428] May [3, 41, 55, 58, 75, 233,234, } \\
236,240,242,247,251-253,272,273,275,276, \\
280,282,285,288,290,291,293,295,296,309, \\
312,315,316,318,320,323,332,429] \text { Put }[15] \\
\text { Req [354, 357, 374, 394, 398, 399] Uca [402, } \\
409,412,420]\end{array}$ & 56 & LC & \\
\hline 78 & Sturnira oporaphilum (Tschudi, 1844) * & $\begin{array}{l}\text { Murciélago de hombros amarillos de } \\
\text { oriente }\end{array}$ & & & & & & & & 1 & Uca [415] & 1 & LC & \\
\hline 79 & Sturnira tildae de la Torre, 1959 & Murciélago de charreteras rojizas & & 1 & 1 & & 1 & 1 & 1 & 1 & $\begin{array}{l}\operatorname{DdM}[342] \text { Lor }[14,22,48,208,347,350,361] \\
\text { May }[1,3,4,76,171,178,180,182,186,187, \\
191,197,203,204,209,212,214,217,220,233- \\
235,241,242,249-253,260,265,270,276,282, \\
285,288,293,296,309,312,314,316,320,429] \\
\text { Put }[23] \text { Req }[355,357,395] \text { Uca [405, 423] }\end{array}$ & 58 & LC & \\
\hline 80 & Uroderma bilobatum Peters, $1866^{* *}$ & Murciélago constructor de toldos & 1 & 1 & 1 & 1 & 1 & & 1 & 1 & $\begin{array}{l}\text { AAm [388, 391] DdM }[25,67,256,343,383] \text { Lor } \\
{[11,208,325,344,345,347,348,361,365,366]} \\
\text { MRC [327, 426] May }[1,4,40,41,46,50,55,63, \\
66,71,75-77,80,83,87,90,139,149,151,158, \\
163,172,179,180,181,183,184,188,189,195, \\
196,197,198,199,202,204,207,209,211,212, \\
217,220,221,222,223,225,231-236,238-240, \\
242,243,247,249-252,260,265,266,269,270, \\
273,275,280,282,285,286,292,293,295,296, \\
305,306,308,309,312,314-316,318,320,322, \\
323,328,433,437] \text { Req }[357,367,395,397-399, \\
411,441] \text { Uca }[405,409,412,415,424]\end{array}$ & 125 & LC & \\
\hline
\end{tabular}


Nombre científico

Nombre común

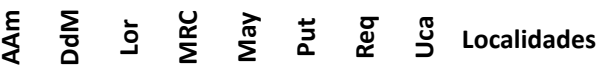

\begin{tabular}{|c|c|c|c|c|c|c|c|c|c|c|c|c|c|c|}
\hline 81 & & Uroderma magnirostrum Davis, 1968 & $\begin{array}{l}\text { Murciélago amarillento constructor } \\
\text { de toldos }\end{array}$ & & & 1 & 1 & 1 & 1 & 1 & 1 & $\begin{array}{l}\text { Lor }[348,350] \text { MRC }[426,428] \text { May }[50,55,64, \\
80,81,83,90,110,130,134,135,140,143,146, \\
149,159,169,173,181,183,184,191,196,202, \\
211,213,220,234,237,247,250-252,276,282, \\
\text { 288, 309, 312, 314, 316, 320, 335] Put [38] Req } \\
\text { [357] Uca [401] }\end{array}$ & 49 & LC \\
\hline 82 & & Vampyressa thyone Thomas, 1909 & $\begin{array}{l}\text { Murciélago de orejas amarillas ecua- } \\
\text { toriano }\end{array}$ & 1 & 1 & 1 & 1 & 1 & 1 & 1 & 1 & $\begin{array}{l}\text { AAm [388] DdM [9, 67, 304, 342, 383] Lor [11, } \\
13,14,22,29,168,170,347,359] \text { MRC [84, } 327] \\
\text { May }[1,3,8,32,40,41,50,63,75,77,179,181, \\
183,195,198,202,207,213,216,218,221,224, \\
226,230,236,239,240,252,260,263,279,280, \\
285,291,295,300,307,310,312,313,315,329, \\
332,437] \text { Put }[15] \text { Req }[355,357,367,394,398, \\
399,411,440-442] \text { Uca }[405,420,425]\end{array}$ & 75 & LC \\
\hline 83 & & Vampyriscus bidens (Dobson, 1878) & Murcielaguito de lista dorsal & 1 & 1 & 1 & & 1 & & 1 & 1 & $\begin{array}{l}\text { AAm [388] DdM [17, 26, 343, 352, 387, 438] Lor } \\
{[20,22,92,325,347,382] \text { May }[3,8,28,46,55} \\
71,75,179,207,212,216,218,221,226,230, \\
240,241,251,252,260,265,269,274,280,281, \\
283,285,287,288,293,309,310,312-314,317 \\
318,320,324,329,435] \text { Req [440] Uca [405] }\end{array}$ & 56 & LC \\
\hline 84 & & Vampyriscus brocki (Peterson, 1968) & Murcielaguito de Brock & & & & 1 & 1 & & 1 & 1 & $\begin{array}{l}\text { MRC [428] May [90, 179, 181, 186, 188, 190, } \\
202,207,212,216,218,247,251,252,263,270, \\
293,305,316,320] \text { Req }[355,357,394,398, \\
399] \text { Uca [425] }\end{array}$ & 27 & LC \\
\hline 85 & & Vampyrodes caraccioli (Thomas, 1889) & Muciélago de listas pronunciadas & & 1 & 1 & & 1 & & 1 & & $\begin{array}{l}\operatorname{DdM}[343,352,353] \text { Lor }[325,337,361,365] \\
\text { May }[1,40,41,46,252,258,320] \text { Req }[374,399 \\
441]\end{array}$ & 17 & LC \\
\hline & \multicolumn{2}{|c|}{ Noctilionidae (2) } & & 1 & 0 & 2 & 2 & 2 & 0 & 1 & 1 & & & \\
\hline 86 & & Noctilio albiventris Desmarest, 1818 & Murciélago pescador menor & 1 & & 1 & 1 & 1 & & 1 & 1 & $\begin{array}{l}\text { AAm [389-391] Lor [35, 321, 325, 337, 361, 363, } \\
365] \text { MRC }[84,327,331] \text { May }[1,4,8,56,94 \\
110,111,125-127,132,139,166,172,182,183 \\
\text { 252] Req }[346,355,357,374] \text { Uca [421] }\end{array}$ & 35 & LC \\
\hline \multirow[t]{2}{*}{87} & & Noctilio leporinus (Linnaeus, 1758) & Murciélago pescador mayor & & & 1 & 1 & 1 & & & & Lor [14] MRC [327] May $[66,114,191,432]$ & 6 & LC \\
\hline & \multicolumn{2}{|c|}{ Furipteridae (1) } & & 0 & 0 & 0 & 0 & 1 & 0 & 1 & 1 & & & \\
\hline \multirow[t]{2}{*}{88} & & Furipterus horrens (F. Cuvier, 1828) & Murciélago sin pulgar & & & & & 1 & & 1 & 1 & May $[63,70]$ Req $[357,374]$ Uca $[418]$ & 5 & LC \\
\hline & \multicolumn{2}{|c|}{ Thyropteridae (4) } & & 0 & 1 & 0 & 3 & 3 & 0 & 4 & 0 & & & \\
\hline 89 & & $\begin{array}{l}\text { Thyroptera discifera (Lichtenstein y Peters, } \\
\text { 1855) * }\end{array}$ & $\begin{array}{l}\text { Murciélago de ventosas de vientre } \\
\text { pardo }\end{array}$ & & & & 1 & 1 & & 1 & & MRC [44] May [141] Req [357] & 3 & LC \\
\hline 90 & & Thyroptera lavali Pine, 1993 & Murciélago de La Val & & & & 1 & 1 & & 1 & & MRC [327] May [180, 186, 272] Req [355] & 5 & LC \\
\hline 91 & & Thyroptera tricolor Spix, 1822 & $\begin{array}{l}\text { Murciélago de ventosas de vientre } \\
\text { blanco }\end{array}$ & & 1 & & 1 & 1 & & 1 & & $\begin{array}{l}\text { DdM }[256] \text { MRC }[44,289,327] \text { May }[8,50,61 \\
70,210,231,232,248,252,263,311,320] \text { Req } \\
{[374,441]}\end{array}$ & 18 & LC \\
\hline 92 & & $\begin{array}{l}\text { Thyroptera wynneae Velazco, Gregorin, Voss y } \\
\text { Simmons, } 2014^{*}\end{array}$ & $\begin{array}{l}\text { Murciélago con alas de disco de Pa- } \\
\text { tricia }\end{array}$ & & & & & & & 1 & & Req [357] & 1 & DD \\
\hline
\end{tabular}




\begin{tabular}{|c|c|c|c|c|c|c|c|c|c|c|c|c|c|}
\hline & Molossidae (13) & & 2 & 1 & 2 & 2 & 12 & 0 & 5 & 2 & & & \\
\hline & Molossinae (13) & & 2 & 1 & 2 & 2 & 12 & 0 & 5 & 2 & & & \\
\hline 93 & Cynomops milleri (Osgood, 1914) * & Murciélago cara de perro de Miller & 1 & & & & & & & & AAm [390] & 1 & LC \\
\hline 94 & Cynomops planirostris (Peters, 1866) * & $\begin{array}{l}\text { Murciélago de cola libre de vientre } \\
\text { blanco }\end{array}$ & & & & & 1 & & & & May $[100,249]$ & 2 & LC \\
\hline 95 & Eumops delticus Thomas, 1923 * & Murciélago de bonete Delta & & & & & 1 & & & & May [165] & 1 & DD \\
\hline 96 & Eumops hansae Sanborn, 1932 * & Murciélago de bonete de Sanborn & & & & & 1 & & 1 & & May $[207,329]$ Req $[398,399]$ & 4 & LC \\
\hline 97 & Eumops maurus (Thomas, 1901) * & Murciélago de bonete de Guyana & & & & & 1 & & & & May [140] & 1 & DD \\
\hline 98 & Eumops trumbulli (Thomas, 1901) * & Murciélago bonetero de los llanos & & & & 1 & 1 & & & & MRC [84] May [43] & 2 & LC \\
\hline 99 & $\begin{array}{l}\text { Molossops neglectus Williams y Genoways, } \\
1980^{*}\end{array}$ & Murciélago cara de perro marrón & & & & & 1 & & 1 & & May $[172,212,303]$ Req $[357]$ & 4 & DD \\
\hline 100 & Molossops temminckii (Burmeister, 1854) * & Murcielaguito de cola libre & & & 1 & & 1 & & & & Lor [361] May $[8,204,252]$ & 4 & $\mathrm{LC}$ \\
\hline 101 & $\begin{array}{l}\text { Molossus alvarezi González-Ruiz, Ramírez- } \\
\text { Pulido \& Arroyo-Cabrales, } 2011 \text { * }\end{array}$ & Murciélago mastín de Álvarez & & & & & 1 & & & & May $[42,50,114]$ & 3 & DD \\
\hline 102 & Molossus coibensis J. A. Allen, 1904 & Murciélago mastín de Coiba & & & & & 1 & & & & May $[97,116,119,120,151]$ & 5 & LC \\
\hline 103 & Molossus molossus (Pallas, 1766) & Murciélago casero & 1 & 1 & 1 & 1 & 1 & & 1 & 1 & 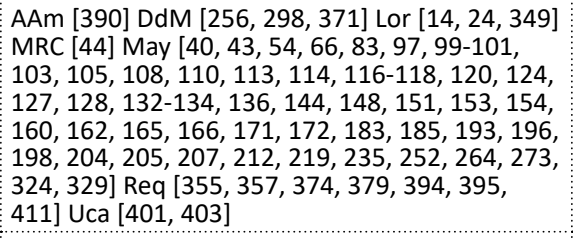 & 70 & LC \\
\hline 104 & Molossus rufus E. Geoffroy, 1805 & Murciélago mastín negro & & & & & 1 & & 1 & 1 & $\begin{array}{l}\text { May }[8,66,104,105,112,113,115,121,136, \\
\text { 138, 154, 172, 212, 252] Req }[357,374,394 \\
\text { 398] Uca [401] }\end{array}$ & 19 & LC \\
\hline 105 & Promops centralis Thomas, 1915 & Murciélago mastín acanelado & & & & & 1 & & 1 & & May $[108,113,212,252] \operatorname{Req}[357]$ & 5 & LC \\
\hline & Vespertilionidae (9) & & 3 & 3 & 4 & 1 & 9 & 1 & 5 & 4 & & & \\
\hline & Vespertilioninae (4) & & 1 & 1 & 1 & 0 & 4 & 0 & 1 & 1 & & & \\
\hline 106 & Eptesicus brasiliensis (Desmarest, 1819) & Murciélago parduzco & & 1 & 1 & & 1 & & 1 & & $\begin{array}{l}\text { DdM [360] Lor [14] May }[66,100,122,144,149 \\
194,252,296] \text { Req }[357,394,400]\end{array}$ & 13 & LC \\
\hline 107 & Eptesicus furinalis (d'Orbigny, 1847) * & Murciélago pardo menor & & & & & 1 & & & & May $[163,251]$ & 2 & LC \\
\hline 108 & Lasiurus blossevillii (Lesson y Garnot, 1826) * & Murciélago rojizo & 1 & & & & 1 & & & & AAm [391] May [159] & 2 & LC \\
\hline 109 & Lasiurus ega (Gervais, 1856) & Murciélago amarillento & & & & & 1 & & & 1 & $\operatorname{May}[66,155,159,252]$ Uca $[401]$ & 5 & LC \\
\hline & Myotinae (5) & & 2 & 2 & 3 & 1 & 5 & 1 & 4 & 3 & & & \\
\hline
\end{tabular}




\begin{tabular}{|c|c|c|c|c|c|c|c|c|c|c|c|c|c|c|c|c|}
\hline & & Nombre científico & Nombre común & $\frac{E}{4}$ & $\sum_{\mathrm{O}}$ & دَ & $\underset{\mathscr{\Psi}}{\Sigma}$ & $\sum^{\frac{\pi}{\pi}}$ & $\vec{z}$ & ठ্ّ & Ј & Localidades & 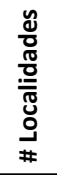 & $\sum_{\mathrm{w}}^{0}$ & בֶ & 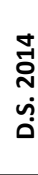 \\
\hline 110 & & Myotis albescens (E. Geoffroy, 1806) & Murcielaguito plateado & 1 & & 1 & & 1 & & 1 & 1 & $\begin{array}{l}\text { AAm }[389,391] \text { Lor }[347,368] \text { May }[1,3,4,8, \\
50,66,70,110,111,116,125,139,166,181 \\
183,184,191,252,273,335] \text { Req }[362,374] \text { Uca } \\
{[393,401]}\end{array}$ & 28 & & LC & \\
\hline 111 & & Myotis keaysi J. A. Allen, 1914 * & Murciélago negruzco & & 1 & & & 1 & & & & $\operatorname{DdM}[341,383]$ May $[255]$ & 3 & & LC & \\
\hline 112 & & Myotis nigricans (Schinz, 1821) & Murciélago negruzco común & 1 & 1 & 1 & 1 & 1 & 1 & 1 & 1 & $\begin{array}{l}\text { AAm [390, 391] DdM }[25,256,373] \text { Lor [14] } \\
\text { MRC }[44] \text { May }[41,50,66,76,80,105,106,114, \\
122,125,129,137,149,150,151,166,172,179, \\
197,223,225,237,243,249,252,255,261,281, \\
287,290,295,315,320,323,329,435] \text { Put }[23] \\
\text { Req }[355,357,374] \text { Uca }[401,405,422]\end{array}$ & 50 & & LC & \\
\hline 113 & & Myotis riparius Handley, 1960 & Murcielaguito acanelado & & & 1 & & 1 & & 1 & & $\begin{array}{l}\text { Lor }[12,347] \text { May }[1,8,50,76,83,88,93,94, \\
115,126,127,152,171,174,178,190,191,194- \\
197,210,235,238,241,251-253,260,265,266, \\
276,316,320,329,330] \text { Req }[355,357,374]\end{array}$ & 41 & & LC & \\
\hline 114 & & Myotis simus Thomas, 1901 & Murciélago vespertino aterciopelado & & & & & 1 & & 1 & 1 & $\begin{array}{l}\text { May }[3,8,61,70,151,162,183,252,329,330] \\
\text { Req }[357] \text { Uca }[401,404,418]\end{array}$ & 14 & & DD & \\
\hline \multicolumn{2}{|c|}{ Total especies (\#) } & 114 & & 33 & 51 & 59 & 46 & 98 & 25 & 91 & 47 & & & & & \\
\hline
\end{tabular}




\section{Literatura citada}

Altringham J, Kerth G. 2016. Chapter 3: Bats and Roads. 2016. Pp. 35-62, en: Voigt CC, Kingston T. (Eds.). 2016. Bats in the Anthropocene: Conservation of Bats in a Changing World. https://doi.org/10.1007/978-3-319-25220-9

Angulo S.R. \& Diaz MM. 2004. Nuevos registros de Sphaeronycteris toxophyllum para la cuenca Amazonica de Peru. Mastozoologia Neotropical 11: 233-236.

Arias-Aguilar A, Hintze F, Aguiar LM, et al. 2018. Who's calling? Acoustic identification of Brazilian bats. Mammal Research 63(3): 231-253. https://doi.org/10.1007/ s13364-018-0367-z

Ascorra CF, Gorchov DL, Cornejo F. 1993. The bats from Jenaro Herrera, Loreto, Perú. Mammalia 57(4): 533-552. https://doi.org/10.1515/mamm.1993.57.4.533

Baker RJ, Solari S, Cirranello A, Simmons NB. 2016. Higher level classification of phyllostomid bats with a summary of DNA synapomorphies. Acta Chiropterologica 18(1): 1-38. https://doi.org/10.3161/15081109A CC2016.18.1.001

Bardales Ordoñez JM. 2015. Diversidad y abundancia de murciélagos (mammalia: Quiroptera) en los bosques del Centro de Investigación y Enseñanza Forestal Puerto Almendra (CIEFOR), Iquitos-Perú.

Bravo A, Borman R. 2008. Mamíferos. Pp: 105-111, en: Alverson WS, Vriesendorp C, del Campo Á, et al. (Eds.) 2008. Ecuador-Perú: Cuyabeno-Güeppí. Rapid Biological and Social Inventories Report 20. The Field Museum, Chicago.

Bravo A, Ríos JA. 2007. Mamíferos Pp. 73-78, en: Vriesendorp C, Álvarez JA, Barbagelata N, et al. (Eds.) 2007. Perú: Nanay, Mazán, Arabela. Rapid Biological Inventories Report 18. The Field Museum, Chicago.

Bravo A. 2010. Mamíferos. Pp 90-96, en: Gilmore MP, Vriesendorp C, Alverson WS, (Eds.). 2010. Perú: Maijuna. Rapid Biological and Social Inventories Report 22. The Field Museum, Chicago

Bravo A, Lizcano DJ, Álvarez-Loayza P. 2016. Mamíferos medianos y grandes. Pp. 140-150, en: Pitman N, Bravo A, Claramunt S, et al. (Eds.) 2016. Perú: Medio Putumayo-Algodón. Rapid Biological and Social Inventories Report 28. The Field Museum, Chicago

Britto B. 2017. Actualización de las Ecorregiones Terrestres de Perú propuestas en el Libro Rojo de Plantas Endémicas del Perú. Gayana. Botánica 74(1): 15-29. https:// doi.org/10.4067/S0717-66432017005000318

Cabrera A. 1958.Catálogo de los mamíferos de América del Sur. Revista del Museo Argentino de Ciencias Naturales "Bernardino Rivadavia" 4(1): 1-307.

Calderón W, Pacheco V. 2012. First report of Artibeus bogotensis Andersen, 1906 (Chiroptera: Phyllostomidae) for Peru. Check List 8(6): 1333-1336. https://doi. org/10.15560/8.6.1333

Castro Vergara L. 2012. Mamíferos. Pp. 127-134, en: Pitman NE, Ruelas I, Alvira D, et al. (Eds.) 2012. Perú: Cerros de Kampankis. Rapid Biological and Social Inventories Report 24. The Field Museum, Chicago

Cevillano SC, Ramos MC. 2011. Nicho alimentario de murciélagos frugívoros en bosque de colina, río Itaya, Loreto Perú. Tesis para obtener el título de Biólogo, Universidad Nacional de la Amazonia Peruana, Iquitos - Perú.

Davis WB, Gardner AL. 2008. Genus Eptesicus. Pp: 440-450. En: Gardner A.L. (Ed). 2008. Mammals of South America Volumen 1 Marsupials, Xenarthrans, Shrews, and Bats.
The University of Chicago Press, Chicago and London.

Díaz MM, Linares García VH. 2012. Refugios naturales y artificiales de Murciélagos (Mammalia: Chiroptera) en la selva baja en el Noroeste de Perú. Gayana 76(2): 117-30. https://doi.org/10.4067/S071765382012000300005

Díaz MM. 2011. New records of bats from the northern region of the Peruvian Amazon. Zoological Research 32(2): 168-178. https://doi.org/10.3724/ SP.J.1141.2011.02168

Dos Reis NR, Peracchi AL, Pedro WA, de Lima IP. (Eds.) 2007. Morcegos do brasil. Univesidade Estadual de Londrina.

Escobedo M, Velazco PM. 2012. First confirmed record for Peru of Diclidurus scutatus Peters, 1869 (Chiroptera: Emballonuridae). Check List 8(3): 554-556. https://doi. org/10.15560/8.3.554

Escobedo M. 2003. Murciélagos. Pp. 82-84. En: Pitman N, Vriesendorp C, Moskovits, et al. (Eds.). 2003. Perú: Yavarí. Field Museum, Environment, Culture, and Conservation.

Escobedo M. 2015. Mamíferos. Pp. 142-151, en: Pitman N, Vriesendorp C, Rivera Chávez L, et al. (Eds.) 2015. Perú: Tapiche-Blanco. Rapid Biological and Social Inventories Report 27. The Field Museum, Chicago.

Fernández-Arellano GJ, Torres-Vásquez MI. 2013. Lista Actualizada de Quirópteros de los Departamentos de Loreto, Ucayali y Madre de Dios (Perú). Barbastella 6(1): 7399. https://doi.org/10.14709/BarbJ.6.1.2013.10

Fonseca RM, Pinto CM. 2004. A new Lophostoma (Chiroptera: Phyllostomidae: Phyllostominae) from the Amazonia of Ecuador. Occasional papers of the Museum of Texas Tech University no. 242:1-9.

Frick WF, Kingston T, Flanders J. 2019. A review of the major threats and challenges to global bat conservation. Annals of the New York Academy of Sciences 1469(1): 5-25. https://doi.org/10.1111/nyas.14045.

Gardner AL. (Ed.) 2008. Mammals of South America Volumen 1 Marsupials, Xenarthrans, Shrews, and Bats. The University of Chicago Press, Chicago and London.

González-Ruiz N, Ramírez-Pulido J, Arroyo-Cabrales J. 2011. A new species of mastiff bat (Chiroptera: Molossidae: Molossus) from Mexico. Mammalian Biology - Zeitschrift Für Säugetierkunde, 76(4), 461-469. https:// doi.org/10.1016/j.mambio.2010.06.004

Hershkovitz P. 1983. Two new species of night monkeys, genus Aotus (Cebidae, Platyrrhini): a preliminary report on Aotus taxonomy. American Journal of Primatology 4(3): 209-243. https://doi.org/10.1002/ ajp.1350040302

Hershkovitz P. 1987. The taxonomy of South American sakis, genus Pithecia (Cebidae, Platyrrhini): A preliminary report and critical review with the description of a new species and a new subspecies. American Journal of Primatology 12(4): 387-468. https://doi. org/10.1002/ajp.1350120402

Hice CL, Solari S. 2002. First record of Centronyceris maximiliani (Fischer, 1929) and two additional records of C. centralis Thomas, 1912 from Peru. Acta Chiropterologica 4(2): 217-220. https://doi. org/10.3161/001.004.0209

Hice CL, Velazco PM, Willig MR. 2004. Bats of the Reserva Nacional Allpahuayo-Mishana, northeastern Peru, with notes on community structure. Acta Chiropterologica 6(2): 319-334. https://doi. 


\section{org/10.3161/1508110042955568}

Hoofer SR, S. Solari S, Larsen PA, et al. 2008. Phylogenetics of the fruit-eating bats (Phyllostomidae: Artibeina) inferred from mitochondrial DNA sequences. Occasional Papers Museum Texas Tech University 277: 1-15.

Hurtado-Miranda NE, Pacheco V. 2014. Análisis filogenético del género Mimon Gray, 1847 (Mammalia, Chiroptera, Phyllostomidae) con la descripción de un nuevo género. Therya 5(3): 751-791.

IUCN. 2012. Categorías y Criterios de la Lista Roja de la UICN: versión 3.1, segunda edición. Disponible en: Https:// portals.iucn.org/library/sites/library/files/documents/RL-2001-001-2nd-Es.pdf

Jorge MLSP, Velazco PM. 2006. Mamíferos. Pp. 98-106, en: Vriesendorp C, Schulenberg TS, Alverson WS, et al. (Eds.) 2006. Perú: Sierra del Divisor. Rapid Biological Inventories Report 17. The Field Museum, Chicago

Koopman KF. 1971. The systematic and historical status of the Florida Eumops (Chiroptera, Molossidae). American Museum novitates; no. 2478. Disponible en: http:// digitallibrary.amnh.org/handle/2246/2686

Ley № 30186. 2014. Ley de Creación de la Provincia de Putumayo en el departamento de Loreto. Diario El Peruano, 6 de mayo de 2014. Disponible en: https:// busquedas.elperuano.pe/download/url/ley-de-creacion-de-la-provincia-de-putumayo-en-el-departamen-ley-n-30186-1079601-3

Lim BK, Engstrom MD, Reid FA, et al. 2010. A new species of Peropteryx (Chiroptera: Emballonuridae) from western Amazonia with comments on phylogenetic relationships within the genus. American Museum Novitates 3686: 1-20. https://doi.org/10.1206/691.1

Linares-Palomino R, Chávez G, Pérez E, et al. 2013. Patrones de diversidad y composición en comunidades de pteridophyta, aves, anfibios, reptiles y murciélagos en la cuenca del río Tapiche, Loreto. Biodiversidad y uso de recursos naturales en la cuenca baja del río Tapiche. Instituto de Investigaciones de la Amazonia Peruana 14-55.

López Wong C. 2013. Mamíferos. Pp. 121-125, en: Pitman N, Ruelas Inzunza E, Vriesendorp C, et al. (Eds.) 2013. Perú: Ere-Campuya-Algodón. Rapid Biological and Social Inventories Report 25. The Field Museum, Chicago.

Ludeña JP, Medina CE. 2017. Segundo registro de Cyttarops alecto y Eumops wilsoni (Mammalia: Chiroptera) en el Perú. Revista Peruana de Biología 24(4): 401-406. https://doi.org/10.15381/rpb.v24i4.14065

Málaga BA, Díaz DR, Arias S, Medina CE. 2020. Una especie nueva de Lasiurus (Chiroptera: Vespertilionidae) del suroeste de Perú. Revista mexicana de biodiversidad, 91. Disponible en: http://revista.ib.unam.mx/index.php/ bio/article/view/3096.

Malo de Molina JA, Velazco S, Pacheco V, Robledo JC. 2011. Análisis de las vocalizaciones del murciélago longirrostro peruano Platalina genovensium Thomas, 1928 (Chiroptera: Phyllostomidae). Revista Peruana de Biología 18(3): 311-318. https://doi.org/10.15381/rpb. v18i3.443

Mantilla-Meluk H, Jimenez-Ortega AM, Baker RJ. 2009. Mammalia, Chiroptera, Phyllostomidae, Lonchophylla pattoni: First record for Ecuador. Investigación, Biodiversidad y Desarrollo 2009; 28 (2): 222-5.

Medina CE, Gregorin R, Zeballos H, et al. 2014. A new species of Eumops (Chiroptera: Molossidae) from southwestern Peru. Zootaxa 3878(1): 19-36. https://doi. org/10.11646/zootaxa.3878.1.2
Medina CE, Lopez E, Pino K, et al. 2015. Biodiversidad de la zona reservada Sierra del Divisor (Perú): una visión desde los mamíferos pequeños. Revista Peruana de Biología 22(2): 199-212. https://doi.org/10.15381/ rpb.v22i2.11354

Mercado Torres A, Galván Meza T, Díaz Salcedo K, et al. 2016. Documento Técnico: Identificación de sitios proritarios para la conservación de la diversidad biológica en el departamento de Loreto. 2016. Disponible en: https://dar.org.pe/archivos/docs/doc_tec_SP_DB.pdf

Meyer CFJ, Struebig MJ, Willig MR. 2016. Chapter 4: Responses of Tropical Bats to Habitat Fragmentation, Logging, and Deforestation. Pp. 63-104, en: Voigt CC, Kingston T. (Eds.). 2016 Bats in the Anthropocene: Conservation of Bats in a Changing World. https://doi. org/10.1007/978-3-319-25220-9_1

Montenegro 0, Escobedo M. 2004. Mamíferos. Pp. 80-87, en: Pitman N, Vriesendorp C, Moskovits, et al. (Eds.). 2003. Perú: Ampiyacu, Apayacu, Yaguas, Medio Putumayo. Rapid Biological Inventories Report 12, The Field Museum, Chicago, 273 pp.

Montenegro 0, Moya L. 2011. Mamíferos. Pp. 126-133, en: Pitman N, Vriesendorp C, Moskovits, et al. (Eds.). 2011. Perú: Yaguas Cotuhé. Rapid Biological and Social Inventories Report 23. The Field Museum, Chicago.

Morales-Martínez DM, Ramírez-Chaves HE, Colmenares-Pinzón JE, Gómez LG. 2020. The Koepcke's spear-nosed bat, Gardnerycteris koepckeae (Gardner and Patton, 1972) (Chiroptera: Phyllostomidae), is not endemic to Peru: first record from the Amazon foothills of Colombia. Mammalia 84(5): 439-447. https://doi. org/10.1515/mammalia-2019-0107

Moras LM, Tavares VDC, Pepato AR, et al. 2016. Reassessment of the evolutionary relationships within the dog-faced bats, genus Cynomops (Chiroptera: Molossidae). Zoologica Scripta 45(5): 465-480. https://doi. org/10.1111/zsc.12169

Moras LG, Gomes AM, Tavares VC. 2014. Distribution and taxonomy of the common big-eared bat Micronycteris microtis (Chiroptera: Phyllostomidae) in South America. Mammalia 79(4): 439-447. https://doi.org/10.1515/ mammalia-2014-0057

Moratelli R, Novaes RLM, Carrión Bonilla C, Wilson DE. 2019. A new species of Myotis (Chiroptera, Vespertilionidae) from Peru. Pp. 239-256. In: Bradley RD, Genoways HH, Schmidly DJ, Bradley LC (eds). From field to laboratory: a memorial volume in honor of Robert J. Baker. Special Publications, Museum of Texas Tech University 71:xi+1-911.

Nogueira MR, Lima IP, Moratelli R, et al. 2014. Checklist of Brazilian bats, with comments on original records. Check List 10(4): 808-821. Disponible en: https://repositorio.inpa.gov.br/bitstream/1/14480/1/artigo-inpa.pdf.

Gobierno Regional de Loreto. Ordenanza Regional $\mathrm{N}^{\circ} 025$ 2016-GRL-CR. Disponible en: http://aplicaciones02. regionloreto.gob.pe/sisdoc/sites/default/files/ normativa_regional/ordenanza_regional_ndeg_0252016-grl-cr.pdf.

Odicio E. 1992. Perfil Demográfico de la Región Loreto, Documento Técnico №1. Instituto de Investigaciones de la Amazonia Peruana, Iquitos, Perú. http://repositorio. iiap.org.pe/bitstream/IIAP/244/2/Odicio_documentotecnico_1992.pdf

Ossa G. 2010. Métodos bioacústicos: una aproximación a la ecología de comunidades de murciélagos en las ecorregiones mediterránea y el bosque templado de Chile. 
Tesis, Grado de Ingeniero Agrónomo. Pontificia Universidad Católica de Chile, Santiago, Chile

Pacheco V, Arias L. 2001. Pp: 85-88, en: Alverson WS, Rodríguez LO, Moskovits DK (Eds.) 2001. Perú: Biabo Cordillera Azul. Rapid Biological Inventories Report 2. Chicago, IL: The Field Museum.

Pacheco V. 2002. Mamíferos del Perú. Pp. 503-550. En: G Ceballos y JA Simonetti, eds. Diversidad y conservación de los mamíferos neotropicales. Comisión Nacional para el Conocimiento y Uso de la Biodiversidad, Mexico DF, Mexico. 582 pp.

Pacheco V, Cadenillas R, Salas E, Tello C, Zeballos H. 2009. Diversidad y endemismo de los mamíferos del Perú. Revista peruana de biología 16(1): 5-32.

Pacheco V, Zevallos A, Cervantes K, et al. 2015. Mamíferos del Refugio de Vida Silvestre Los Pantanos de Villa, Lima-Perú. Científica 12(1): 26-41. https://doi. org/10.21142/cient.v12i1.163.

Pacheco V, Inche B, Wust W. 2018. Mamíferos del Perú. Grupo SA La República, eds. Animales sorprendentes. Editorial Septiembre S.A.C., Lima. Pp. 1-120.

Pacheco V, Pacheco J, Zevallos A, et al. 2020. Mamíferos pequeños de humedales de la costa central del Perú. Revista peruana de biología 27(4): 483-498. https://doi. org/10.15381/rpb.v27i4.19204

Pacheco V, Diaz S, Graham-Angeles L, Flores-Quispe M, Calizaya-Mamani G, Ruelas D, Sánchez-Vendizú P. 2021. Lista actualizada de la diversidad de los mamíferos del Perú y una propuesta para su actualización. Revista peruana de biología 28(4): e21019 001 - 030 (Noviembre 2021). doi: http://dx.doi.org/10.15381/rpb. v28i4.21019

Parlos JA, Timm RM, Swier VJ, et al. 2014. Evaluation of the paraphyletic assemblage within Lonchophyllinae, with description of a new tribe and genus. Occasional papers of the Museum of Texas Tech University 320:i + 1-23.

Patterson BD y López Wong C. 2014. Mamíferos. Pp: 154-167, en: Pitman N, Vriesendorp C, Alvira D, et al. (Eds.) 2014. Perú: Cordillera Escalera-Loreto. Rapid Biological and Social Inventories No. 26. Field Museum of Natural History.

Patton JL, Pardiñas UF, D’Elía G. (Eds.). 2015. Mammals of South America, volume 2: rodents. University of Chicago Press.

Pirlot P. 1968. Chiroptères du Perou, specialement de hauteAmazonie. Mammalia 32:86-96.

Pitman N, Gagliardi-Urrutia G, Jenkins CN. 2013. La biodiversidad de Loreto, Perú: El conocimiento actual de plantas y vertebrados terrestres. Center for International Environmental Law. Disponible en: https://www. researchgate.net/publication/271505773_La_biodiversidad_de_Loreto_Peru_El_conocimiento_actual_de_ plantas_y_vertebrados_terrestres.

Quintana H, Pacheco V. 2007. Identificación y distribución de los murciélagos vampiros del Perú. Revista Peruana de Medicina Experimental y Salud Pública 24(1): 81-88. https://doi.org/10.17843/rpmesp.2007.241.1084

Quintana H, Pacheco V, Salas E. 2009. Diversidad y conservación de los mamíferos de Ucayali, Perú. Ecología aplicada 8(1-2): 91-103.

Ramírez-Chaves HE, Noguera-Urbano EA, Rodríguez-Posada ME. 2013. Mamíferos (Mammalia) del departamento de Putumayo, Colombia. Revista de la Academia Colombiana de Ciencias Exactas, Físicas y Naturales
37(143): 263-286.

Ramos MC, Cevillano SC, Aquino R, et al. 2017. Diversidad de murciélagos en bosques de colina del Río Itaya, Loreto, Perú. Folia Amazónica 26(2): 139-152. https://doi. org/10.24841/fa.v26i2.430

Ramos-Rodriguez M, Falcón Ayapi R, Díaz Vázquez R. 2018. Murciélagos indicadores de hábitats perturbados en la Reserva Nacional Allpahuayo Mishana, Perú. Folia Amazónica 27(1): 31-46. https://doi.org/10.24841/ fa.v27i1.444

Regalado J \& Albuja Viteri LH. 2012. Ampliación de la distribución de Sturnira nana y Lophostoma brasiliense en el Ecuador.

Rengifo EM, Pacheco V, Salas E. 2011. An additional record of Platyrrhinus ismaeli Velazco, 2005 on the western slope of Peru, with taxonomic comments. Chiroptera Neotropical 17(1): 903-907.

Rivera MD, Vallejo AF, Tinajero JG, Burneo SF. 2019. Biblioteca de llamadas de ecolocación de los murciélagos del Ecuador. En: Brito J, Camacho MA, Romero V, Vallejo AF. (Eds). Mamíferos del Ecuador. Versión 2019.0. Museo de Zoología, Pontificia Universidad Católica del Ecuador. (Accedido el 06 feb 2021). Disponible en: https:// bioweb.bio/faunaweb/mammaliaweb/Ecolocacion/

Rodríguez-San Pedro A, Allendes J, Ossa G. 2016. Updated list of bats of Chile with comments on taxonomy, ecology, and distribution. Biodiversity and Natural History, 2(1), 16-39. Disponible en: http://www.biodiversnathist.com/index.php/bnh/article/view/31

Ruelas D, López E. 2018. Análisis morfogeométrico de las especies peruanas de Carollia (Chiroptera: Phyllostomidae). Mastozoología Neotropical 25(2): 419-438. https://doi.org/10.31687/saremMN.18.25.2.0.03

Ruelas DC, Pacheco V. 2015. Taxonomía y distribución de Vampyriscus brocki (Peterson 1968; Phyllostomidae: Chiroptera) en Perú y primer registro para Ucayali. Therya 6(3): 625-642. https://doi.org/10.12933/ therya-15-302

Saavedra SM, Villalobos RG. 2010. Dispersión de semillas por murciélagos (Mammalia: Chiroptera) en bosque primario, bosque secundario y sistemas de cultivo en la Reserva Forestal Santa Cruz, río Mazán. Tesis para obtener el título de Biólogo, Universidad Nacional de la Amazonia Peruana, Iquitos - Perú.

Sanborn CC. 1949b. Mammals from the Rio Ucayali, Peru. J. Mammal. 30:277-88.

Schipper J, Chanson JS, Chiozza F, et al. 2008. The status of the world's land and marine mammals: diversity, threat, and knowledge. Science 322(5899): 225-230.

Servicio Nacional de Áreas Naturales Protegidas. https://www. sernanp.gob.pe/el-sinanpe\#

Simmons NB, Voss RS, Fleck DW. 2002. A new Amazonian species of Micronycteris (Chiroptera: Phyllostomidae) with notes on the roosting behavior of sympatric congeners. American Museum Novitates 2002(3358): 1-16. https://doi.org/10.1206/00030082(2002)358<0001:ANASOM>2.0.CO;2

Solari S, Baker RJ. 2006. Mitochondrial DNA sequence, karyotypic, and morphological variation in the Carollia castanea species complex (Chiroptera: Phyllostomidae) with description of a new species. Texas Tech University. Natural Science Research Laboratory.

Solari S, Van Den Bussche RA, Hoofer SR, Patterson BD. 2004. Geographic distribution, ecology, and phylo- 
genetic affinities of Thyroptera lavali Pine 1993. Acta Chiropterologica 6(2): 293-302. https://doi. org/10.3161/1508110042955496

Stephens L, Traylor Jr MA. 1983. Ornithological gazetteer of Peru. Harvard University. 270 pp. https://doi. org/10.5962/bhl.title.14634

Tello C, DG Streicker, J Gomez, PM Velazco. 2013. New records of pigmentation disorders in molossid and phyllostomid (Chiroptera) bats from Peru. Mammalia 78(2): 191197. https://doi.org/10.1515/mammalia-2013-0019

Thomas 0. 1924.0n a collection of mammals made by $\mathrm{Mr}$. Latham Rutter in the Peruvian Amazons. The Annals and magazine of natural history

Thomas 0. 1927a.The Godman-Thomas Expedition to Peru. V. Mammals collected by Mr. R. W. Hendee in the province of San Martin, N. Peru, mostly at Yurac Yacu. The Annals and magazine of natural history ser.9,19: 361-375.

Thomas 0. 1928d.The Godman-Thomas Expedition to Peru. VIII. On mammals obtained by Mr. Hendee at Pebas and Iquitos, upper Amazons. The Annals and magazine of natural history ser.10, 2: 285-94.

Thompson CW, Phelps KL., Allard MW, et al. 2021. Preserve a Voucher Specimen! The Critical Need for Integrating Natural History Collections in Infectious Disease Studies. Mbio: 12(1).

Toffoli R, Boano G, Bonardi A, et al. 2018. Contribution to the bats knowledge of the Pacaya Samiria National Reserve, Loreto, Peru (Mammalia Chiroptera). Biodiversity Journal 29(4): 351-356.

Ugarte-Núñez JA. 2020. Clave de identificación por ecolocación de 20 especies de murciélagos del suroeste de Perú. Ciencia \& Desarrollo (27): 37-48.

Valqui M. 2001. Mammal diversity and ecology of terrestrial small rodents in Western Amazonia. Tesis Doctoral. University of Florida.

Vásquez Újina FA. 2019. Murciélagos indicadores de perturbación del área de concesión para la conservación en la cuenca alta del río Itaya y zonas aledañas perturbadas, Loreto-Perú

Velazco P, Gardner AL. 2009. A new species of Platyrrhinus (Chiroptera: Phyllostomidae) from western Colombia and Ecuador, with emended diagnoses of P. aquilus, P. dorsalis, and P. umbratus. Proceedings of the Biological Society of Washington 122(3): 249-281. https:// doi.org/10.2988/08-40.1

Velazco PM, Patterson BD. 2014. Two new species of yellowshouldered bats, genus Sturnira Gray, 1842 (Chiroptera, Phyllostomidae) from Costa Rica, Panama and western Ecuador. ZooKeys (402): 43-66. https://doi. org/10.3897/zookeys.402.7228.

Velazco PM, Patterson BD. 2019. Small mammals of the Mayo River Basin in Northern Peru, with the description of a new species of Sturnira (Chiroptera: Phyllostomidae). Bulletin of the American Museum of Natural History 2019(429): 1-70.

Velazco PM, Simmons NB. 2011. Systematics and taxonomy of great striped-faced bats of the genus Vampyrodes Thomas, 1900 (Chiroptera: Phyllostomidae). American Museum Novitates 3710: 1-35. Disponible en: http://digitallibrary.amnh.org/handle/2246/6104

Velazco PM. 2005. Morphological phylogeny of the bat genus Platyrrhinus Saussure, 1860 (Chiroptera: Phyllostomidae) with the description of four new species. Fieldiana
Zoology 1(105): 1-53. https://doi.org/10.3158/00150754(2005)105[1:MPOTBG]2.0.CO;2

Velazco PM, Gardner AL, Patterson BD. 2010. Systematics of the Platyrrhinus helleri species complex (Chiroptera: Phyllostomidae), with descriptions of two new species. Zoological Journal of the Linnean Society 159(3): 785-812.

Velazco PM, Gregorin R, Voss RS, Simmons NB. 2014. Extraordinary local diversity of disk-winged bats (Thyropteridae: Thyroptera) in northeastern Peru, with the description of a new species and comments on roosting behavior. American Museum Novitates 2014(3795): 1-28.

Velazco PM., Soto-Centeno A, Fleck DW, et al. 2017. A New Species of Nectar-feeding Bat of the Genus Hsunycteris (Phyllostomidae: Lonchophyllinae) from Northeastern Peru. American Museum Novitates 3881: 1-26. https://doi.org/10.1206/3881.1

Velazco PM, Voss RS, Fleck DW \& Simmons NB. 2021. Mammalian Diversity and Matses Ethnomammalogy in Amazonian Peru Part 4: Bats. Bulletin of the American Museum of Natural History 451(1): 1-200. https:// doi.org/10.1206/0003-0090.451.1.1

Voigt CC, Kingston T. 2016. Chapter 1: Bats in the Anthropocene. Pp 1-9, en: Voigt CC, Kingston T. (Eds.). Bats in the Anthropocene: Conservation of Bats in a Changing World. https://doi.org/10.1007/978-3-319-25220-9

Voss RS, DW Fleck, Strauss RE, et al. 2016. Roosting ecology of Amazonian bats: evidence for guild structure in hyperdiverse mammalian communities. American Museum Novitates 3870: 1-44. https://doi. org/10.1206/3870.1

Voss RS, Fleck DW. 2017. Mammalian Diversity and Matses Ethnomammalogy, in Amazonian Peru, Part 2: Xenartha, Carnivora, Perisodactyla, Artiodactyla and Sirenia. Bulletin of the American Museum of Natural History 417: 1-118. https://doi.org/10.1206/00030090-417.1.1

Willey RH. 2010. Alfonso Olalla and his family: the ornithological exploration of Amazonian Peru. Bulletin of the American Museum of Natural History 343: 1-68. https://doi.org/10.1206/677.1

Wilson DE, Ascorra CF, Solari S. 1996. Bats as indicators of habitat disturbance. Pp: 577-592. En: Wilson D.E. \& A. Sandoval (Eds.). 1996. Manu, the Biodiversity of Southeastern Peru. Smithsonian Institution, Ed. Horizonte. Lima, Perú.

Zeballos Patrón H, Pacheco V, Baraybar L. 2001. Diversidad y conservación de los mamíferos de Arequipa, Perú. Revista peruana de biología 8(2): 094-104. https://doi. org/10.15381/rpb.v8i2.6564

Zegarra O, Pacheco J, Pacheco V. 2020. Distributional patterns of the Brazilian free-tailed bat Tadarida brasiliensis in the Peruvian territory. Therya 11(3): 495. 
Agradecimientos / Acknowledgments:

A los miembros del departamento de Mastozoología del Museo de Historia Natural por el apoyo en la identificación de los especímenes, a Daniel Llancachahua-Tarqui y Jaime Pacheco por sus aportes y comentarios que ayudaron a mejorar este manuscrito y a Biosfera Consultores Ambientales S.A.C.

\section{Conflicto de intereses / Competing interests:}

VP es editor de la RPB, no participo en el proceso de edición del artículo. Los autores no incurren en conflictos de intereses.

\section{Rol de los autores / Authors Roles:}

LG-A: Conceptualización, Investigación, Escritura- Preparación del borrador original, Redacción: revisión y edición. PS-V: Investigación, Redacción: revisión y edición. SD: Investigación, Redacción: revisión y edición. VP: Investigación, Curación de datos, Redacción: revisión y edición.

Fuentes de financiamiento / Funding:

Biosfera Consultores Ambientales S.A.C. por el contrato BIOCAM 503-2020

\section{Aspectos éticos / legales; Ethics / legals:}

Los autores declaran no haber incurrido en aspectos antiéticos ni haber omitido normas legales en la realización de este trabajo. 
Anexo 1. Lista de localidades incluidas en los análisis y sus coordenadas

\begin{tabular}{|c|c|c|c|}
\hline Pto. & Localidad & Grados X & Grados $Y$ \\
\hline 1 & Maynas, Torres Causana, Río Lagartococha, Campamento Catalino & -75.259600 & -0.528270 \\
\hline 2 & Maynas, Torres Causana, Lagartococha River mouth & -75.261389 & -0.655000 \\
\hline 3 & Maynas, Torres Causana, Puesto de vigilancia Castaña, Río Aguarico & -75.240000 & -0.803610 \\
\hline 4 & Maynas, Torres Causana, Puesto de vigilancia Arcadia, Río Napo & -74.666500 & -1.709240 \\
\hline 5 & Maynas, Torres Causana, 1-2 km E P. V. Arcadia, Río Napo/ "Platanal" & -74.671600 & -1.726130 \\
\hline 6 & Putumayo, Rosa Panduro, Panduro River (= Rosa Panduro) & -73.411700 & -1.789440 \\
\hline 7 & Maynas, Torres Causana, $12 \mathrm{Km}$ S Puesto de vigilancia Arcadia (en cuevas) & -74.692700 & -1.791020 \\
\hline 8 & Maynas, Napo, Río Curaray (= Curaray River mouth) & -74.090555 & -2.363333 \\
\hline 9 & Datem del Marañón, Andoas, Andoas A & -76.116335 & -2.408862 \\
\hline 10 & Loreto , Trompeteros, Shiviyacu CV4 & -76.087606 & -2.488440 \\
\hline 11 & $\begin{array}{l}\text { Loreto, Trompeteros, Lote } 1 \mathrm{AB} \text {, Shiviyacu. Altura del km } 78 \text { de la carretera a San Jacinto. Redes al lado } \\
\text { de una quebrada }\end{array}$ & -76.095405 & -2.491148 \\
\hline 12 & $\begin{array}{l}\text { Loreto, Trompeteros, Lote } 1 \mathrm{AB} \text {, Carretera de Tnte. López a Shiviyacu paralelo a una quebrada y carretera } \\
\text { a } 200 \mathrm{~m} \text { del Cv } 5\end{array}$ & -76.115751 & -2.568884 \\
\hline 13 & Loreto, Trompeteros, Tnte. López CV5 & -76.114048 & -2.575054 \\
\hline 14 & Loreto, Trompeteros, Teniente López & -76.116667 & -2.583333 \\
\hline 15 & Putumayo, Yaguas, Choro & -71.485750 & -2.610611 \\
\hline 16 & $\begin{array}{l}\text { Datem del Marañón, Andoas, Lote } 1 \mathrm{AB} \text {, Capahuari Norte, Altura del km } 20 \text { de la carretera Capahuari } \\
\text { Norte. Redes en al lado de una quebrada, en una trocha ancha }\end{array}$ & -76.497360 & -2.673880 \\
\hline 17 & Datem del Marañón, Andoas, Capahuari norte & -76.500633 & -2.674066 \\
\hline 18 & $\begin{array}{l}\text { Loreto, Trompeteros, Lote } 1 \mathrm{AB} \text {, Jíbaro. } 0.5 \mathrm{~km} \text { del Puerto Jíbaro paralelas al rio corrientes, una quebradi- } \\
\text { ta y al Cv } 6\end{array}$ & -76.026911 & -2.701024 \\
\hline 19 & Loreto, Trompeteros, Jibarito CV7 & -76.076853 & -2.704237 \\
\hline 20 & $\begin{array}{l}\text { Loreto, Trompeteros, Lote } 1 \mathrm{AB} \text {. en el km } 33 \text { de la carretera Jibarito - Teniente López. Paralelo a la carre- } \\
\text { tera y al Cv } 7\end{array}$ & -76.077672 & -2.705276 \\
\hline 21 & Loreto, Trompeteros, Tambo este CV3 & -76.316349 & -2.706785 \\
\hline 22 & Loreto, Trompeteros, Jibaro CV6 & -76.029134 & -2.713035 \\
\hline 23 & Putumayo, Yaguas, Cachimbo & -70.529194 & -2.718306 \\
\hline 24 & $\begin{array}{l}\text { Loreto, Trompeteros, Lote } 1 \mathrm{AB} \text {. Al termino sur de las líneas de flujo de Jibarito, paralelo a la carretera y } \\
\text { al Cv } 8\end{array}$ & -76.016348 & -2.771688 \\
\hline 25 & Datem del Marañón, Andoas, Andoas B & -76.452500 & -2.803611 \\
\hline 26 & $\begin{array}{l}\text { Datem del Marañón, Andoas, Lote } 1 \mathrm{AB} \text {, Capahuari Sur, Altura del km } 10.5 \text { de la carretera Tambo. Redes } \\
\text { colocadas al lado de la carretera en una colina alta }\end{array}$ & -76.339986 & -2.804133 \\
\hline 27 & Loreto, Trompeteros, Tambo este CV2, redes cruzan una quebrada & -76.320534 & -2.807673 \\
\hline 28 & Maynas, Alto Nanay, Quebrada Agua Negra & -74.978550 & -2.854120 \\
\hline 29 & Loreto, Trompeteros, Lote $1 \mathrm{AB}$, Jibarito sur; redes al lado de una quebradita dentro del pm21 & -76.005058 & -2.855840 \\
\hline 30 & Putumayo, Yaguas, Yaguas & -71.415028 & -2.864861 \\
\hline 31 & Maynas, Alto Nanay, Quebrada Agua Blanca & -74.808170 & -2.926980 \\
\hline 32 & Maynas, Napo, Lower Rio Napo region, E bank Río Yanayacu, ca. 90 km N Iquitos & -73.247222 & -2.934160 \\
\hline 33 & Datem del Marañón, Morona, Comunidad Panintza, Río Situche & -77.427384 & -3.041105 \\
\hline 34 & Maynas, Mazán, 1.5 km S Libertad, S bank Río Napo, 80 km N Iquitos & -73.333330 & -3.046900 \\
\hline 35 & Loreto, Tigre, Campamento Patria Nueva & -75.275240 & -3.096780 \\
\hline 36 & Putumayo, Yaguas, Río Morona & -71.285470 & -3.149680 \\
\hline 37 & Datem del Marañón, Andoas, Naranjal & -76.410000 & -3.170000 \\
\hline 38 & Putumayo, Yaguas, Alto Cotuhé & -70.899028 & -3.198778 \\
\hline
\end{tabular}




\begin{tabular}{|c|c|c|c|}
\hline Pto. & Localidad & Grados X & Grados $Y$ \\
\hline 39 & Maynas, Alto Nanay, Lote 123, Sector 1 , Comunidad Nativa Alvarenga & -74.702430 & -3.210340 \\
\hline 40 & Maynas, Mazán, Sucusari, Quebrada Grande/ Quebrada Sucusari, Explonapo Camp & -72.917380 & -3.257210 \\
\hline 41 & $\begin{array}{l}\text { Maynas, Mazán, Sucusari, left bank of Napo river, } 70 \text { km NE Iquitos, Amazon Center for Environmental } \\
\text { Education and Research (ACEER) }\end{array}$ & -72.918708 & -3.266317 \\
\hline 42 & Maynas, Mazán, Quebrada Yanamono & -72.900000 & -3.266667 \\
\hline 43 & Maynas, Alto Nanay, Alto Río Nanay & -74.622960 & -3.305780 \\
\hline 44 & Mariscal Ramón Castilla, Pebas, Estiron, Ampiyacu River & -71.850000 & -3.316667 \\
\hline 45 & Datem del Marañón, Andoas, Sabaloyacu A & -76.310000 & -3.330000 \\
\hline 46 & Maynas, Alto Nanay, Río Nanay, Santa Luisa & -74.583333 & -3.333330 \\
\hline 47 & Mariscal Ramón Castilla, Pebas, Pebas & -71.816667 & -3.333333 \\
\hline 48 & Loreto, Trompeteros, Jibarito CV8 & -76.007781 & -3.416833 \\
\hline 49 & Maynas, Alto Nanay, A 17.35 km norte de la localidad de Albarenga & -74.404760 & -3.435930 \\
\hline 50 & Maynas, Indiana, Yanamono, Quebrada Yanamono, aprox 60 km NE Iquitos & -72.843600 & -3.448610 \\
\hline 51 & Maynas, Las Amazonas, 1 km N Río Napo, 157 river km NNE Iquitos & -72.750000 & -3.457620 \\
\hline 52 & Maynas, Mazán, La Libertad & -73.268628 & -3.458797 \\
\hline 53 & Maynas, Mazán, Nuevo Varaderillo A & -73.088556 & -3.471889 \\
\hline 54 & Maynas, Mazán, Nuevo Varaderillo B & -73.099500 & -3.473333 \\
\hline 55 & Maynas, Las Amazonas, Quebrada Orán, 5 km N Río Amazonas, 85 km NE Iquitos & -72.517000 & -3.475000 \\
\hline 56 & Maynas, Las Amazonas, Isla Pasto, Río Amazonas, opposite Aysana, 80 km NE Iquitos (= Isla Orán) & -72.535061 & -3.476769 \\
\hline 57 & Maynas, Mazán, Nuevo Varaderillo C & -73.096528 & -3.481306 \\
\hline 58 & Maynas, Punchana, Punto Alegre & -73.423233 & -3.481984 \\
\hline 59 & Maynas, Alto Nanay, A 13.6 km noroeste de la localidad de Albarenga & -74.322540 & -3.485230 \\
\hline 60 & Maynas, Punchana, Río Momón & -73.430768 & -3.486193 \\
\hline 61 & Maynas, Las Amazonas, Monte Alegre, Apayacu River/ Apayacu, Amazon River & -72.181944 & -3.488889 \\
\hline 62 & Maynas, Indiana, Indiana, Chacra de Clayder & -73.039080 & -3.494130 \\
\hline 63 & Maynas, Indiana, S Rio Amazonas, ca. 10 km SSW mouth Rio Napo on E bank Quebrada Vainilla & -72.819820 & -3.498480 \\
\hline 64 & Maynas, Mazán, Mazan River, west tributary to Ucayali River & -73.097417 & -3.498556 \\
\hline 65 & Maynas, Punchana, Almirante Guise & -73.442810 & -3.499840 \\
\hline 66 & Maynas, Indiana, Puerto Indiana, Río Amazonas & -73.044444 & -3.502778 \\
\hline 67 & Datem del Marañón, Andoas, Sabaloyacu B & -76.270000 & -3.520000 \\
\hline 68 & Loreto, Trompeteros, Pavayacu & -75.423662 & -3.522086 \\
\hline 69 & Loreto, Trompeteros, Capirona & -75.413443 & -3.522489 \\
\hline 70 & Maynas, Las Amazonas, Orosa, Río Amazonas & -72.189444 & -3.525278 \\
\hline 71 & Maynas, Alto Nanay, Lote 123 , Sector 4, Comunidad Nativa Alvarenga & -74.425540 & -3.527260 \\
\hline 72 & Datem del Marañón, Andoas, Soplín & -76.280000 & -3.530000 \\
\hline 73 & Datem del Marañón, Andoas, Loboyacu & -76.260000 & -3.550000 \\
\hline 74 & Maynas, Punchana, Grau de Pampa Hermosa & -73.367837 & -3.586728 \\
\hline 75 & Maynas, Indiana, Río Manití, Santa Cecilia & -72.957028 & -3.605841 \\
\hline 76 & Maynas, Punchana, Iquitos Picuro Yacu & -73.268330 & -3.618060 \\
\hline 77 & Maynas, Punchana, Barrio Orosa Estación Madreselva II, río Amazonas & -72.240100 & -3.627740 \\
\hline 78 & Maynas, Belén, Iquitos, Padre Isla, Caracocha (laguna) & -73.153300 & -3.637790 \\
\hline 79 & Maynas, Punchana, Santa Rosa & -73.314261 & -3.642942 \\
\hline 80 & Maynas, Belén, Iquitos, Padre Isla & -73.158584 & -3.650404 \\
\hline 81 & Maynas, Punchana, Padrecocha /Asentamiento Bambú, Pasaje Los Cedros-Bambú & -73.285330 & -3.691500 \\
\hline
\end{tabular}




\begin{tabular}{|c|c|c|c|}
\hline Pto. & Localidad & Grados X & Grados $Y$ \\
\hline 82 & Maynas, Punchana, Padrecocha, $30 \mathrm{~m}$ de la carretera Shihua & -73.295433 & -3.692400 \\
\hline 83 & Maynas, Punchana, Padrecocha (ciudad) & -73.281000 & -3.697080 \\
\hline 84 & Mariscal Ramón Castilla, San Pablo, Río Amazonas, boca Río Peruate & -71.483333 & -3.700000 \\
\hline 85 & Maynas, Punchana, Río Nanay, Puerto Transelva & -73.271717 & -3.700950 \\
\hline 86 & Maynas, Punchana, 10 miles below Iquitos, junction of Nanay and Amazon Rivers & -73.248778 & -3.701028 \\
\hline 87 & Maynas, Punchana, 5 km NNE Iquitos, $122 \mathrm{~m}$ & -73.234367 & -3.703647 \\
\hline 88 & Maynas, Punchana, Punchana, Bellavista Nanay (puerto) & -73.249483 & -3.703850 \\
\hline 89 & Maynas, Punchana, Bellavista Nanay Av. 11 de Abril & -73.246280 & -3.708050 \\
\hline 90 & Maynas, Punchana, Comunidad de Manacamiri, Río Nanay, Fundo Morropón A & -73.301030 & -3.708530 \\
\hline 91 & Maynas, Punchana, Comunidad de Manacamiri, Río Nanay, Fundo Morropón B & -73.302833 & -3.709900 \\
\hline 92 & Loreto, Tigre, Cerca de Río Tigre & -74.539346 & -3.712031 \\
\hline 93 & Maynas, Punchana, Punchana, Facultad de Medicina, Universidad de la Amazonia Peruana & -73.258980 & -3.721030 \\
\hline 94 & Maynas, Punchana, Recreo Don Pepe, al lado del cementerio de Punchana & -73.255850 & -3.722750 \\
\hline 95 & Maynas, Punchana, Asentamiento Humano Pilar Nores, $400 \mathrm{~m}$ E. del Hospital Regional de Loreto & -73.251670 & -3.722820 \\
\hline 96 & Loreto, Tigre, Lote 123 , Sector 13 , San Andres & -74.478120 & -3.724510 \\
\hline 97 & Maynas, Punchana, Punchana, Calle Amazonas 1086 (casa de Dr. Gendrau) & -73.251883 & -3.725100 \\
\hline 98 & Maynas, Punchana, Iquitos - Punchana Av. Colonial, Distrito de Punchana & -73.257700 & -3.725750 \\
\hline 99 & Maynas, Punchana, Camal Municipal de Punchana & -73.249183 & -3.726133 \\
\hline 100 & Maynas, Punchana, Villa del Ejército (entre Av. 28 de Julio-Freyre y calle Piura) & -73.243467 & -3.727633 \\
\hline 101 & Maynas, Punchana, Hogar "Padre Angel Rodríguez" (INABIF), Av. 28 de Julio Nro 500 & -73.245717 & -3.727700 \\
\hline 102 & Maynas, Punchana, Iquitos Iglesia Inmaculada-Punchana & -73.242590 & -3.728580 \\
\hline 103 & Maynas, Punchana, CEI Centro Educativo Inicial Nro 165, República Federal de Alemania, Club de Leones & -73.245070 & -3.728800 \\
\hline 104 & Maynas, Punchana, Iglesia Inmaculada & -73.243000 & -3.728967 \\
\hline 105 & Maynas, Iquitos, Iquitos Moronacocha: Aserradero-pradera, frente al Conafovicer & -73.271350 & -3.732230 \\
\hline 106 & Maynas, lquitos, Río Nanay, Santa Rita & -73.322800 & -3.732500 \\
\hline 107 & Maynas, Iquitos, lquitos, Pevas 1460 & -73.253450 & -3.739250 \\
\hline 108 & Maynas, Iquitos, Iquitos, Parque Zonal de Iquitos, entre calle Pevas y Yavari & -73.249820 & -3.739950 \\
\hline 109 & Maynas, Iquitos, Iquitos, Coliseo Cerrado de Iquitos & -73.253917 & -3.744050 \\
\hline 110 & Maynas, Iquitos, Iquitos Oeste, CESM (Centro Educativo San Martín) “Loreto” & -73.262833 & -3.744950 \\
\hline 111 & Maynas, Iquitos, Moronacocha (curva), lecho del Lago Moronacocha & -73.266680 & -3.745280 \\
\hline 112 & Maynas, Iquitos, Iquitos, Calle Nanay & -73.246230 & -3.745670 \\
\hline 113 & Maynas, Belén, Casa Pevas 354 & -73.243190 & -3.746130 \\
\hline 114 & Maynas, lquitos, Iquitos A & -73.247220 & -3.748060 \\
\hline 115 & Maynas, Iquitos, Boulevar, Calle Malecón-Maldonado, frente al Seminario San Agustín & -73.242180 & -3.748870 \\
\hline 116 & Maynas, Iquitos, Iquitos, Edificio EsSalud (calles Raymondi-Napo) & -73.243550 & -3.749030 \\
\hline 117 & Maynas, Iquitos, Iquitos B & -73.259460 & -3.749270 \\
\hline 118 & Maynas, Iquitos, Iquitos, Huallaga cuadra dos & -73.246480 & -3.749680 \\
\hline 119 & $\begin{array}{l}\text { Maynas, Iquitos, Colegio Primario Secundario "RADM" (Rosa Agustina Donayre de Morey), calle Putuma- } \\
\text { yo (Iquitos) }\end{array}$ & -73.252283 & -3.749950 \\
\hline 120 & $\begin{array}{l}\text { Maynas, Iquitos, Iquitos Colegio Primario Secundario "RADM" (Rosa Agustina Donayre de Morey), calle } \\
\text { Putumayo }\end{array}$ & -73.252283 & -3.749950 \\
\hline 121 & Maynas, Iquitos, Iquitos, "Centro de Idiomas de la UNAP," Sargento Lores 446 & -73.247320 & -3.749950 \\
\hline 122 & Maynas, Iquitos, Iquitos Moronacocha, casa, Jr. Gonzales Vigil & -73.269070 & -3.750920 \\
\hline 123 & Maynas, Iquitos, Pueblo Joven Micaela Bastida (entre las calles Cahuide y Javier Erau) & -73.272020 & -3.751930 \\
\hline 124 & Maynas, Iquitos, Iquitos (Bermudez 1004) & -73.254833 & -3.752100 \\
\hline
\end{tabular}




\begin{tabular}{|c|c|c|c|}
\hline Pto. & Localidad & Grados X & Grados Y \\
\hline 125 & Maynas, Iquitos, Iquitos, Boulevard, orillas del Río Itaya & -73.237030 & -3.752480 \\
\hline 126 & Maynas, Iquitos, Boulevar, Puerto Salaverry, Calle Malecón, Tarapacá s/n & -73.244120 & -3.752770 \\
\hline 127 & Maynas, Belén, Provincia de Maynas, departamento de Loreto (3 45.17' S; 73ำ 14.65' O) & -73.244167 & -3.752833 \\
\hline 128 & Maynas, Iquitos, Iquitos, Calle Roman nro. 988 & -73.255600 & -3.752850 \\
\hline 129 & Maynas, Iquitos, Iquitos Túpac Amaru, Lourdes de Leon \#510 & -73.269610 & -3.754480 \\
\hline 130 & Maynas, Iquitos, Iquitos Túpac Amaru & -73.277210 & -3.755200 \\
\hline 131 & Maynas, Iquitos, Asentamiento Urbano El Porvenir & -73.277830 & -3.755330 \\
\hline 132 & Maynas, Iquitos, Pampachica (Bungalow), 2.1 km SW de la Av. Quiñones y carretera a Pampachica & -73.275200 & -3.756450 \\
\hline 133 & Maynas, Iquitos, Pampa Chica, Iquitos & -73.272138 & -3.757152 \\
\hline 134 & Maynas, Iquitos, Iquitos, calle Fanning última cuadra & -73.257520 & -3.760970 \\
\hline 135 & Maynas, Iquitos, Asentamiento Pacaya Samiria "Complejo Deportivo SUTUNAP" (calle San Lorenzo) & -73.273200 & -3.762400 \\
\hline 136 & Maynas, Iquitos, Iquitos, Villa de técnicos de la FAP (Fuerza Aérea de Perú), calle Moore última cuadra & -73.256583 & -3.762517 \\
\hline 137 & Maynas, Iquitos, Iquitos Pasaje Huascar-Belen & -73.245470 & -3.763030 \\
\hline 138 & Maynas, Iquitos, Hospital Iquitos César Garayar García & -73.254071 & -3.763215 \\
\hline 139 & Maynas, Iquitos, Villa Victoria, INDECO (calle Pihuicho 109) & -73.277420 & -3.764900 \\
\hline 140 & Maynas, San Juan Bautista, Asociación de Viviendas 15 de Mayo (E Av. Quiñones) & -73.295417 & -3.765050 \\
\hline 141 & Maynas, Belén, Loreto, Iquitos & -73.250000 & -3.766667 \\
\hline 142 & Maynas, Iquitos, Club Oriental Amazónico (COA) & -73.272350 & -3.768230 \\
\hline 143 & Maynas, San Juan Bautista, Policlínico de la Policía Nacional del Perú, Av. A. Quiñones & -73.277250 & -3.768650 \\
\hline 144 & Maynas, Iquitos, Estación Experimental IVITA & -73.274800 & -3.769750 \\
\hline 145 & Maynas, Iquitos, Complejo Recreacional Amazónico & -73.290380 & -3.770950 \\
\hline 146 & Maynas, Iquitos, Comité La Coruña & -73.298400 & -3.775350 \\
\hline 147 & Maynas, San Juan Bautista, Recreo Las Camelias, app 200 m de la Av. Quiñones km 4.7 & -73.292720 & -3.776250 \\
\hline 148 & Maynas, Iquitos, Rumococha, 3.3 km W de Av. Quiñones 4700 & -73.313217 & -3.777517 \\
\hline 149 & Maynas, San Juan Bautista, Iquitos-San Juan; Casa de Sra. Soledad & -73.291490 & -3.777570 \\
\hline 150 & Maynas, Iquitos, San Juan Avenida de la Participación & -73.278790 & -3.778520 \\
\hline 151 & Maynas, Belén, Moena Caño & -73.224917 & -3.778800 \\
\hline 152 & Maynas, Iquitos, Comité Simón Bolívar, Fundo Ruth, Calle Flores Lote 17, carretera a Santa Clara & -73.304330 & -3.779700 \\
\hline 153 & Maynas, Iquitos, Recreo, Calle Begonias s/n & -73.289000 & -3.780820 \\
\hline 154 & Maynas, Iquitos, Asentamiento Secada, IEPS Francisco Secada Vignetta (colegio) & -73.294870 & -3.781370 \\
\hline 155 & Maynas, Iquitos, Iquitos San Juan, casa, Psje. Las Begonias & -73.289220 & -3.781860 \\
\hline 156 & Maynas, Iquitos, Caserío Santa Sofía, río Nanay (playa) & -73.356700 & -3.784000 \\
\hline 157 & Maynas, Iquitos, Iquitos San Juan: Granja Lunar & -73.297630 & -3.785080 \\
\hline 158 & Maynas, San Juan Bautista, CaseBarrio Cabo López Casa de Julio & -73.264410 & -3.786230 \\
\hline 159 & Maynas, Iquitos, km 1 de la carretera Iquitos-Nauta, "Club Social Sanmartinense" & -73.299330 & -3.786430 \\
\hline 160 & Maynas, Iquitos, Estación Experimental Agraria San Roque & -73.294917 & -3.786950 \\
\hline 161 & Maynas, Iquitos, Río Nanay, San Pablo Cuyana & -73.366562 & -3.787179 \\
\hline 162 & Maynas, Iquitos, Asentamiento Héroes de Cenepa, Fundo Gallo Giro & -73.286300 & -3.787400 \\
\hline 163 & Maynas, Iquitos, km 1 carretera Iquitos (casa Sixto Mananita) & -73.299730 & -3.790880 \\
\hline 164 & Maynas, Iquitos, km 1 carretera Santo Tomás (Empresa Eimerson) & -73.305400 & -3.791020 \\
\hline 165 & $\begin{array}{l}\text { Maynas, San Juan Bautista, Piscina Olímpica del Instituto Peruano de Deportes, km } 1.5 \text { al W de la carre- } \\
\text { tera Iquitos-Nauta }\end{array}$ & -73.303850 & -3.794933 \\
\hline 166 & $\begin{array}{l}\text { Maynas, Iquitos, Santo Tomás pocos km fuera (WSW) Iquitos camino debajo de carretera Iquitos-Nauta, } \\
\text { El Portero }\end{array}$ & -73.312910 & -3.796670 \\
\hline 167 & Maynas, San Juan Bautista, Iquitos Avicola San Miguel, km 3 carretera Iquitos-Nauta & -73.311470 & -3.807300 \\
\hline
\end{tabular}




\begin{tabular}{|c|c|c|c|}
\hline Pto. & Localidad & Grados X & Grados $Y$ \\
\hline 168 & Loreto, Trompeteros, Nueva Unión & -75.009862 & -3.807678 \\
\hline 169 & Maynas, San Juan Bautista, Santo Tomás, 6 km al W del km 1 de la carretera Iquitos-Nauta & -73.338130 & -3.809680 \\
\hline 170 & Loreto, Trompeteros, Lote 8 de Pluspetrol & -75.075592 & -3.815717 \\
\hline 171 & Maynas, San Juan Bautista, Quistococha km 5 carretera Iquitos-Nauta, parque Quistococha & -73.319770 & -3.815800 \\
\hline 172 & Maynas, San Juan Bautista, Quistococha km 4.5 carretera Iquitos-Nauta, PEA(IIAP), lado O del camino & -73.319956 & -3.817058 \\
\hline 173 & $\begin{array}{l}\text { Maynas, San Juan Bautista, Quistococha km } 5 \text { carretera Iquitos-Nauta, } 1 \text { km O del camino, Fundo Quisto- } \\
\text { cocha (Amigo de Arturo) }\end{array}$ & -73.332030 & -3.817490 \\
\hline 174 & Maynas, Belén, Iquitos Fundo Chávez (Río Amazonas) & -73.199400 & -3.821210 \\
\hline 175 & Maynas, San Juan Bautista, km 6 de la carretera Iquitos-Nauta, 700 m W camino a Zungarococha & -73.331800 & -3.824600 \\
\hline 176 & Maynas, San Juan Bautista, Quistococha, Iquitos & -73.318330 & -3.827780 \\
\hline 177 & Maynas, San Juan Bautista, Puerto Almendra & -73.376520 & -3.827840 \\
\hline 178 & Maynas, Belén, Mazanillo & -73.219517 & -3.828083 \\
\hline 179 & Maynas, San Juan Bautista, Zungarococha Casa en Av. Miraflores & -73.356170 & -3.828140 \\
\hline 180 & Maynas, San Juan Bautista, Zungarococha, 5.4 km al W del km 6 de la carretera Iquitos-Nauta & -73.371467 & -3.830417 \\
\hline 181 & Maynas, San Juan Bautista, Zungarococha Casa en Av. Corrientillo & -73.361230 & -3.831350 \\
\hline 182 & $\begin{array}{l}\text { Maynas, San Juan Bautista, Centro de Investigación y Enseñanza Forestal (CIEFOR) Puerto Almendras - } \\
\text { Universidad Nacional de la Amazonia Peruana }\end{array}$ & -73.375278 & -3.831667 \\
\hline 183 & Maynas, San Juan Bautista, Km 2.5 camino a Zungarococha, Fundo Shushuna & -73.336560 & -3.831980 \\
\hline 184 & $\begin{array}{l}\text { Maynas, San Juan Bautista, Corrientillo, km } 6 \text { de la carretera Iquitos-Nauta, } 4.5 \text { km W camino a Zungaro- } \\
\text { cocha }\end{array}$ & -73.362500 & -3.832583 \\
\hline 185 & Maynas, Belén, Quistococha & -73.266667 & -3.833333 \\
\hline 186 & Maynas, San Juan Bautista, Arboretum/Zungarococha & -73.373040 & -3.833710 \\
\hline 187 & Maynas, San Juan Bautista, Zungarococha, $6.5 \mathrm{~km}$ al $W$ del km 6 de la carretera Iquitos-Nauta & -73.377220 & -3.833980 \\
\hline 188 & Maynas, San Juan Bautista, Zungarococha, $5.2 \mathrm{~km}$ al $\mathrm{W}$ del km 6 de la carretera Iquitos-Nauta & -73.368280 & -3.834570 \\
\hline 189 & Maynas, San Juan Bautista, Comunidad Puerto Alegría, Río Itaya & -73.306050 & -3.835530 \\
\hline 190 & Maynas, San Juan Bautista, Puerto Almendra Arboretum de CEIFOR & -73.374550 & -3.838940 \\
\hline 191 & Maynas, San Juan Bautista, Ninarumi, 7.4 km al W del km 6 de la carretera Iquitos-Nauta & -73.380780 & -3.841720 \\
\hline 192 & Maynas, San Juan Bautista, Ninarumi, 8.4 km al W del km 6 de la carretera Iquitos-Nauta & -73.385933 & -3.842933 \\
\hline 193 & $\begin{array}{l}\text { Maynas, San Juan Bautista, Ninarumi, Colegio Nro 60778, } 7.9 \text { km al W del km } 6 \text { de la carretera Iquitos- } \\
\text { Nauta }\end{array}$ & -73.385033 & -3.843083 \\
\hline 194 & Maynas, San Juan Bautista, Ninarumi banco del Río Nanay & -73.391480 & -3.845460 \\
\hline 195 & Maynas, San Juan Bautista, Ninarumi, 7.4 km al W y $500 \mathrm{~m}$ al SE del km 6 de la carretera Iquitos-Nauta & -73.380217 & -3.845517 \\
\hline 196 & Maynas, San Juan Bautista, Los Delfines, km 9 de la carretera Iquitos-Nauta & -73.339730 & -3.847480 \\
\hline 197 & Maynas, San Juan Bautista, Zungarococha 2.5 km S, King Kong; Fundo Manuela Vásquez & -73.362710 & -3.847530 \\
\hline 198 & Maynas, Belén, Centro Unión, up Río Aucaya from Iquitos (16km SE Iquitos) & -73.113859 & -3.848637 \\
\hline 199 & Maynas, San Juan Bautista, Ninarumi, $7.4 \mathrm{~km}$ al W y 1 km al SE del km 6 de la carretera Iquitos-Nauta & -73.373933 & -3.849833 \\
\hline 200 & Maynas, San Juan Bautista, Peña Negra km 10 carretera Iquitos-Nauta & -73.346720 & -3.853850 \\
\hline 201 & Maynas, Belén, Río Itaya & -73.306896 & -3.854832 \\
\hline 202 & Maynas, San Juan Bautista, Peña Negra, 600 m al W del km 10 de la carretera Iquitos-Nauta & -73.345150 & -3.855367 \\
\hline 203 & Maynas, San Juan Bautista, Llanchama, sendas de chacra detrás de la casa de Arnold Sanchez Perez & -73.409720 & -3.857220 \\
\hline 204 & Maynas, San Juan Bautista, Nina Rumi & -73.392481 & -3.859385 \\
\hline 205 & Maynas, San Juan Bautista, CaseBarrio Llanchama, entrada a Zungarococha & -73.403580 & -3.860690 \\
\hline 206 & Maynas, San Juan Bautista, Peña Negra km 11.5 carretera Iquitos-Nauta, área Marshy & -73.340020 & -3.864410 \\
\hline 207 & Maynas, San Juan Bautista, Llanchama & -73.400746 & -3.864682 \\
\hline 208 & Loreto, Trompeteros, Lote 8 & -75.012627 & -3.866147 \\
\hline 209 & Maynas, San Juan Bautista, Peña Negra, 200 m al E del km 10,7 de la carretera Iquitos-Nauta & -73.332950 & -3.866150 \\
\hline
\end{tabular}




\begin{tabular}{|c|c|c|c|}
\hline Pto. & Localidad & Grados X & Grados Y \\
\hline 210 & Maynas, San Juan Bautista, Peña Negra, 800 m al E del km 11 de la carretera Iquitos-Nauta & -73.335550 & -3.873270 \\
\hline 211 & Maynas, San Juan Bautista, Varillal, 1.6 km W del km 13.6 de la carretera Iquitos-Nauta & -73.358950 & -3.874300 \\
\hline 212 & Maynas, San Juan Bautista, Llanchama, cerca al Puesto de Control El Varillal & -73.403185 & -3.874471 \\
\hline 213 & Maynas, San Juan Bautista, Varillal, 1.8 km W del km 13.6 de la carretera Iquitos-Nauta & -73.362900 & -3.877170 \\
\hline 214 & Maynas, San Juan Bautista, Mishana, río Nanay, banco sur, transecto de Chris & -73.485820 & -3.878270 \\
\hline 215 & Maynas, San Juan Bautista, Mishana, río Nanay, banco sur & -73.492520 & -3.879940 \\
\hline 216 & Maynas, San Juan Bautista, Mishana, río Nanay, banco sur, casa de Pedro/Sergio & -73.492520 & -3.879940 \\
\hline 217 & Maynas, San Juan Bautista, Varillal, 2 km W del km 13.6 de la carretera Iquitos-Nauta & -73.366850 & -3.880030 \\
\hline 218 & $\begin{array}{l}\text { Maynas, San Juan Bautista, Mishana río arriba desde pueblo en banco sur, río Nanay, senda de la izquier- } \\
\text { da }\end{array}$ & -73.491350 & -3.880580 \\
\hline 219 & Maynas, San Juan Bautista, Mishana, río Nanay, banco sur, Casa de Chris Hice & -73.491730 & -3.880620 \\
\hline 220 & Maynas, San Juan Bautista, Varillal, 400 m W 200 m N del km 14 de la carretera Iquitos-Nauta & -73.354800 & -3.882483 \\
\hline 221 & Maynas, San Juan Bautista, Mishana & -73.492400 & -3.882570 \\
\hline 222 & Maynas, San Juan Bautista, Cerca Peña Negra, km 12 SO carretera a Nauta & -73.331840 & -3.883080 \\
\hline 223 & Maynas, San Juan Bautista, Varillal km 14 carretera Iquitos-Nauta, lado O del camino, Fundo El Aguajal & -73.350050 & -3.883920 \\
\hline 224 & Maynas, San Juan Bautista, Mishana, río Nanay, banco sur, chacra de Manuel & -73.491850 & -3.883980 \\
\hline 225 & Maynas, San Juan Bautista, Varillal & -73.350306 & -3.887306 \\
\hline 226 & $\begin{array}{l}\text { Maynas, San Juan Bautista, Mishana río Nanay, banco sur, río arriba desde pueblo cerca de la chacra de } \\
\text { Pedro }\end{array}$ & -73.501040 & -3.890110 \\
\hline 227 & Maynas, San Juan Bautista, Mishana, río Nanay, banco sur, cercanías chacra de Pedro & -73.500170 & -3.890710 \\
\hline 228 & Maynas, San Juan Bautista, Mishana, río Nanay, banco sur, Tahuampa & -73.500170 & -3.890710 \\
\hline 229 & Maynas, San Juan Bautista, Mishana, río Nanay, banco sur, chacra de Eva & -73.494540 & -3.893510 \\
\hline 230 & Maynas, San Juan Bautista, Mishana, río Nanay, banco sur, cercanías chacra de Eva & -73.491330 & -3.897240 \\
\hline 231 & Maynas, Belén, Estación Biológica Isla Muyuy, Amazon river, 20 km SE Iquitos, Comunidad de Santa Ana & -73.228055 & -3.900000 \\
\hline 232 & Maynas, San Juan Bautista, Cerca a la Aldea, km 18 SO carretera a Nauta & -73.370660 & -3.904460 \\
\hline 233 & Maynas, San Juan Bautista, Moralillo, 1.5 km E 500 m S del km 15.2 de la carretera Iquitos-Nauta & -73.343620 & -3.906320 \\
\hline 234 & Maynas, San Juan Bautista, Moralillo, 1.5 km E 400 m S del km 15.2 de la carretera Iquitos-Nauta & -73.343070 & -3.909050 \\
\hline 235 & Maynas, San Juan Bautista, Moralillo, 2 km E del km 15.2 de la carretera Iquitos-Nauta & -73.337970 & -3.909470 \\
\hline 236 & Maynas, San Juan Bautista, Fundo Mery Rojas km 19.7 carretera Iquitos-Nauta, 10 min. caminando NO & -73.382690 & -3.913420 \\
\hline 237 & Maynas, San Juan Bautista, Fundo Mery Rojas km 19.7 carretera Iquitos-Nauta, lado O del camino & -73.377330 & -3.920060 \\
\hline 238 & Maynas, San Juan Bautista, km 19 de la carretera Iquitos-Nauta & -73.375083 & -3.923817 \\
\hline 239 & Maynas, San Juan Bautista, El Dorado Fundo Las Galeras, km 5 carretera Iquitos-Nauta & -73.381390 & -3.924040 \\
\hline 240 & Maynas, San Juan Bautista, Mishana, 34 km WSW Iquitos & -73.466667 & -3.933333 \\
\hline 241 & Maynas, San Juan Bautista, km 22,7 de la carretera Iquitos-Nauta & -73.395170 & -3.939520 \\
\hline 242 & Maynas, San Juan Bautista, Otorongo km 21 carretera Iquitos-Nauta, 1.5 km E del camino & -73.371460 & -3.942310 \\
\hline 243 & Maynas, San Juan Bautista, El Dorado km 23.5 carretera Iquitos-Nauta, recreo Encanto de Anaconda & -73.397060 & -3.942710 \\
\hline 244 & Maynas, San Juan Bautista, El Otorongo-El Milagro km 21 carretera Iquitos-Nauta & -73.371320 & -3.945320 \\
\hline 245 & Maynas, San Juan Bautista, El Dorado km 23 carretera Iquitos-Nauta, frente a El Dorado & -73.408380 & -3.951240 \\
\hline 246 & $\begin{array}{l}\text { Maynas, San Juan Bautista, Mishana km } 25 \text { carretera Iquitos-Nauta, Reserva Allpahuayo-Mishana, que- } \\
\text { brada pasando Frutales }\end{array}$ & -73.431510 & -3.952060 \\
\hline 247 & Maynas, San Juan Bautista, Mishana km 25 carretera Iquitos-Nauta, Reserva Allpahuayo-Mishana & -73.420520 & -3.955400 \\
\hline 248 & $\begin{array}{l}\text { Maynas, San Juan Bautista, Mishana km } 25 \text { carretera Iquitos-Nauta, Reserva Allpahuayo-Mishana, senda } \\
\text { a Frutales }\end{array}$ & -73.412640 & -3.960130 \\
\hline 249 & $\begin{array}{l}\text { Maynas, San Juan Bautista, El Dorado km } 25.5 \text { carretera Iquitos-Nauta, E del camino, Noriega Montero } \\
\text { (Chacra) }\end{array}$ & -73.409500 & -3.960810 \\
\hline 250 & Maynas, San Juan Bautista, El Dorado, km 25 de la carretera Iquitos-Nauta, app. 500 m al E & -73.399070 & -3.961650 \\
\hline
\end{tabular}




\begin{tabular}{|c|c|c|c|}
\hline Pto. & Localidad & Grados $\mathrm{X}$ & Grados Y \\
\hline 251 & Maynas, San Juan Bautista, 1 km E km 25.3 de la carretera Iquitos-Nauta (Fundo San Martín) & -73.404317 & -3.965900 \\
\hline 252 & $\begin{array}{l}\text { Maynas, San Juan Bautista, Centro de Investigaciones de la Reserva Nacional Allpahuayo Mishana, km } \\
26.8 \text { de la carretera Iquitos-Nauta }\left(S 3^{\circ} 58^{\prime}, W 73^{\circ} 25^{\prime}\right)\end{array}$ & -73.416667 & -3.966667 \\
\hline 253 & Maynas, Iquitos, El Dorado, km 25 de la carretera Iquitos-Nauta, app. 1.5 km al E & -73.393667 & -3.966833 \\
\hline 255 & Maynas, San Juan Bautista, 25 km S de Iquitos, Estación Biológica Allpahuayo & -73.417000 & -3.967000 \\
\hline 256 & Datem del Marañón, Morona, Río Morona, Quebrada Pushaga & -77.213641 & -3.972166 \\
\hline 257 & $\begin{array}{l}\text { Maynas, San Juan Bautista, } 24 \text { de Junio km } 27 \text { carretera Iquitos-Nauta E del camino, casa de Edward } \\
\text { Silva }\end{array}$ & -73.419900 & -3.977700 \\
\hline 258 & Maynas, San Juan Bautista, km 28, SO carretera a Nauta & -73.412410 & -3.982600 \\
\hline 259 & Maynas, San Juan Bautista, Cerca Allpahuayo, km 28 SO carretera a Nauta & -73.414280 & -3.983440 \\
\hline 260 & Maynas, San Juan Bautista, Caserío Palo Seco, app.500 m E km 28.8 de la carretera Iquitos-Nauta & -73.415333 & -3.987117 \\
\hline 261 & Maynas, San Juan Bautista, Trece de febrero km 30.5 carretera Iquitos-Nauta, 1.5 km NO del camino & -73.437740 & -3.989920 \\
\hline 262 & Maynas, San Juan Bautista, Caserío Palo Seco, 2.9 km E del km 28.8 de la carretera Iquitos-Nauta & -73.405983 & -3.995817 \\
\hline 263 & Maynas, San Juan Bautista, Trece de febrero km 31.5, SO carretera a Nauta. UNAP & -73.449610 & -3.996230 \\
\hline 264 & Maynas, San Juan Bautista, Trece de febrero km 31.5 carretera Iquitos-Nauta, Estación de campo UNAP & -73.441870 & -3.999710 \\
\hline 265 & Maynas, San Juan Bautista, El Paujil, 1.8 km al W del km 35 de la carretera Iquitos-Nauta & -73.446450 & -4.020283 \\
\hline 266 & Maynas, San Juan Bautista, 13 de Febrero, Fundo Nemith, E km 33 de la carretera Iquitos-Nauta & -73.429600 & -4.025400 \\
\hline 267 & Maynas, San Juan Bautista, Trece de febrero km 34 carretera Iquitos-Nauta, lado S & -73.432930 & -4.029640 \\
\hline 268 & Maynas, San Juan Bautista, Trece de febrero km 34 SO carretera a Nauta & -73.428600 & -4.036580 \\
\hline 269 & Maynas, San Juan Bautista, El Paujil km 37 carretera Iquitos-Nauta, 1.04 km NO del camino & -73.445580 & -4.049060 \\
\hline 270 & Maynas, San Juan Bautista, Paujil, W km 37.45 de la carretera Iquitos-Nauta & -73.442250 & -4.058780 \\
\hline 271 & $\begin{array}{l}\text { Maynas, San Juan Bautista, Comunidad Zona Centro Ex Petroleros, km } 41 \text { Carretera Iquitos-Nauta, app } 5 \\
\text { km W }\end{array}$ & -73.479200 & -4.061400 \\
\hline 272 & Maynas, San Juan Bautista, Nuevo Horizonte, km 39.5 SO carretera a Nauta & -73.456850 & -4.073920 \\
\hline 273 & Maynas, San Juan Bautista, Ex Petroleros, $300 \mathrm{~m} \mathrm{~W} \mathrm{~km} 39.8$ de la carretera Iquitos-Nauta & -73.453000 & -4.079000 \\
\hline 274 & Maynas, Alto Nanay, A 20 km sur de la localidad de Diamante Azul & -73.770840 & -4.082250 \\
\hline 275 & Maynas, San Juan Bautista, Nuevo Horizonte km 41 carretera Iquitos-Nauta, lado E & -73.448320 & -4.089080 \\
\hline 276 & Maynas, San Juan Bautista, San Lucas, W km 43 de la carretera Iquitos-Nauta & -73.463183 & -4.104117 \\
\hline 277 & Maynas, San Juan Bautista, km 44, SO carretera a Nauta & -73.451170 & -4.118160 \\
\hline 278 & Maynas, San Juan Bautista, El Triunfo Km 46 carretera Iquitos-Nauta, E del camino & -73.467490 & -4.132750 \\
\hline 279 & Maynas, San Juan Bautista, Km 47 carretera Iquitos-Nauta & -73.460750 & -4.144740 \\
\hline 280 & Maynas, San Juan Bautista, El Triunfo km 48.6 carretera Iquitos-Nauta, O del camino & -73.489870 & -4.147180 \\
\hline 281 & Maynas, San Juan Bautista, El Triunfo km 48 carretera Iquitos-Nauta B & -73.467390 & -4.150520 \\
\hline 282 & Maynas, San Juan Bautista, El Triunfo km 48.3 carretera Iquitos-Nauta, O del camino & -73.474170 & -4.151400 \\
\hline 283 & Maynas, San Juan Bautista, El Triunfo Km 48 carretera Iquitos-Nauta A & -73.479970 & -4.154130 \\
\hline 284 & Maynas, San Juan Bautista, El Triunfo Km 48.5 carretera Iquitos-Nauta, O del camino & -73.469220 & -4.154600 \\
\hline 285 & Maynas, San Juan Bautista, El Triunfo km 48.8 carretera Iquitos-Nauta O del camino & -73.475970 & -4.155600 \\
\hline 286 & Maynas, San Juan Bautista, Cerca El Triunfo, km 49 SO carretera a Nauta & -73.484620 & -4.155670 \\
\hline 287 & Maynas, San Juan Bautista, La Habana km 49.5 carretera Iquitos-Nauta O del camino & -73.482620 & -4.165570 \\
\hline 288 & Maynas, San Juan Bautista, La Habana km 47 carretera Iquitos-Nauta E del camino & -73.460700 & -4.169300 \\
\hline 289 & Mariscal Ramón Castilla, Yavarí, Río Yavarí Mirim, San Fernando & -70.284246 & -4.170245 \\
\hline 290 & Maynas, San Juan Bautista, La Habana Km 52 carretera Iquitos-Nauta, 1.1 km O del camino & -73.483720 & -4.179660 \\
\hline 291 & Maynas, San Juan Bautista, La Habana Km 52 carretera Iquitos-Nauta, 1 km NO del camino & -73.481030 & -4.181120 \\
\hline 292 & Maynas, San Juan Bautista, La Habana km 52 carretera Iquitos-Nauta O del camino B & -73.477420 & -4.181800 \\
\hline 293 & Maynas, San Juan Bautista, La Habana km 52 carretera Iquitos-Nauta E del camino & -73.471930 & -4.185020 \\
\hline
\end{tabular}




\begin{tabular}{|c|c|c|c|}
\hline Pto. & Localidad & Grados X & Grados Y \\
\hline 294 & Maynas, San Juan Bautista, La Habana km 52 carretera Iquitos-Nauta & -73.468420 & -4.187830 \\
\hline 295 & Maynas, San Juan Bautista, Habanillo km 53 carretera Iquitos-Nauta & -73.480560 & -4.191690 \\
\hline 296 & Maynas, San Juan Bautista, La Habana km 52 carretera Iquitos-Nauta O del camino A & -73.478130 & -4.193380 \\
\hline 297 & Datem del Marañón, Morona, Campamento Cabecera Quebrada Wee (C4) B & -77.567520 & -4.199840 \\
\hline 298 & Datem del Marañón, Morona, Mayuriaga B & -77.304917 & -4.202194 \\
\hline 299 & Datem del Marañón, Morona, Campamento Cabecera Quebrada Wee (C4) A & -77.527250 & -4.205840 \\
\hline 300 & Maynas, San Juan Bautista, km 55, SO carretera a Nauta & -73.487870 & -4.208340 \\
\hline 301 & Datem del Marañón, Morona, Nuevo San Martín & -77.239056 & -4.223278 \\
\hline 302 & Datem del Marañón, Morona, Mayuriaga A & -77.239028 & -4.224444 \\
\hline 303 & Maynas, San Juan Bautista, Cahuide km 57 carretera Iquitos-Nauta casa de Bertha Gomez & -73.492170 & -4.226200 \\
\hline 304 & Datem del Marañón, Pastaza, Huangana, aprox. 7.25 Kms al NO de la boca del Río Pastaza & -76.568500 & -4.237470 \\
\hline 305 & Maynas, San Juan Bautista, Caserío Cahuide km 59 carretera Iquitos-Nauta & -73.491990 & -4.242310 \\
\hline 306 & Maynas, San Juan Bautista, Caserío Cahuide km 60, 1 Km NO del camino & -73.501260 & -4.244380 \\
\hline 307 & Maynas, San Juan Bautista, Caserío Cahuide km 60, 0.35 Km NO del camino & -73.494980 & -4.246760 \\
\hline 308 & Maynas, San Juan Bautista, Caserío Cahuide km 60.4 carretera Iquitos-Nauta, O del camino & -73.499340 & -4.248280 \\
\hline 309 & Maynas, San Juan Bautista, Belgica km 60.4 carretera Iquitos-Nauta O del camino & -73.495230 & -4.248330 \\
\hline 310 & Maynas, San Juan Bautista, Belgica km 61.6 carretera Iquitos-Nauta O del camino & -73.509530 & -4.255680 \\
\hline 311 & Maynas, San Juan Bautista, Cahuide km 60 SO carretera a Nauta & -73.485880 & -4.256610 \\
\hline 312 & Maynas, San Juan Bautista, Belgica km 62 carretera Iquitos-Nauta E del camino & -73.496920 & -4.257000 \\
\hline 313 & Maynas, San Juan Bautista, Belgica km 62 carretera Iquitos-Nauta, alcantarillas debajo del camino & -73.501410 & -4.257270 \\
\hline 314 & Maynas, San Juan Bautista, Belgica km 62 carretera Iquitos-Nauta O del camino & -73.507920 & -4.262370 \\
\hline 315 & Maynas, San Juan Bautista, Cahuide km 61 carretera Iquitos-Nauta & -73.501450 & -4.262680 \\
\hline 316 & Maynas, San Juan Bautista, Concesión para la Conservación Cuenca Alta del Río Itaya (CCCARI) & -73.633619 & -4.265258 \\
\hline 317 & Maynas, San Juan Bautista, A 20.4 km oeste de la localidad de Nueva Villa Belen & -73.788240 & -4.267670 \\
\hline 318 & Maynas, San Juan Bautista, Belgica km 63.7 carretera Iquitos-Nauta E del camino & -73.505880 & -4.279000 \\
\hline 319 & Datem del Marañón, Morona, 54 km. al NO de la boca del río Morona & -77.237780 & -4.280830 \\
\hline 320 & Maynas, San Juan Bautista, Cuenca alta del río Itaya & -73.705661 & -4.281169 \\
\hline 321 & Loreto, Tigre, Boca de R Tigre & -74.317000 & -4.283000 \\
\hline 322 & Maynas, San Juan Bautista, km 66.5 SO carretera a Nauta & -73.525780 & -4.300890 \\
\hline 323 & Maynas, San Juan Bautista, km 67.5 carretera Iquitos-Nauta & -73.517130 & -4.302070 \\
\hline 324 & Maynas, Fernando Lores, Quebrada Blanco, Comunidad de Limón & -73.183333 & -4.316667 \\
\hline 325 & Loreto, Nauta, Río Tigre, 1 km above Río Tigrillo & -74.279353 & -4.329265 \\
\hline 326 & Mariscal Ramón Castilla, Yavarí, Nazareth (=Amelia) frente a Remate de Males & -70.201441 & -4.333250 \\
\hline 327 & Mariscal Ramón Castilla, Yavarí, Río Yavarí Mirim, Quebrada Esperanza & -71.916667 & -4.333333 \\
\hline 328 & Maynas, San Juan Bautista, Caserío La Paz km 70 carretera Iquitos-Nauta & -73.541030 & -4.337090 \\
\hline 329 & $\begin{array}{l}\text { Maynas, Fernando Lores, Estación Biológica Quebrada Blanco 2, Communal Reserve Tamshiyacu-Tahua- } \\
\text { yo, ca. } 70 \text { km SSE Iquitos }\end{array}$ & -73.150000 & -4.350000 \\
\hline 330 & Maynas, Fernando Lores, Estación Biológica Quebrada Blanco 1 & -73.159800 & -4.352700 \\
\hline 331 & Mariscal Ramón Castilla, Yavarí, Río Yavarí Mirim, San Vicente & -72.491800 & -4.363630 \\
\hline 332 & Maynas, San Juan Bautista, km 75 SO carretera a Nauta & -73.553080 & -4.366270 \\
\hline 333 & Datem del Marañón, Manseriche, Puerto Melendez, Maranon River & -77.516667 & -4.450000 \\
\hline 334 & $\begin{array}{l}\text { Mariscal Ramón Castilla, Yavarí, Margen izquierdo Río Yavari en la desembocadura del lago Preto (Conce- } \\
\text { sión para la conservación Lago Preto Paredon). }\end{array}$ & -71.716667 & -4.500000 \\
\hline 335 & Maynas, Fernando Lores, Río Yarapa, Albergue Yacumama, SE Iquitos (= Río Yarapa) & -73.366667 & -4.516667 \\
\hline 336 & Datem del Marañón, Manseriche, Comunidad Nativa Nuevo progreso & -77.497960 & -4.522573 \\
\hline
\end{tabular}




\begin{tabular}{|c|c|c|c|}
\hline Pto. & Localidad & Grados X & Grados Y \\
\hline 337 & Loreto, Nauta, Nauta, Río Samiria & -73.550000 & -4.533333 \\
\hline 338 & Loreto, Urarinas, San Antonio de Bancal, aprox. 6 km al NO de la boca del Río Urituyacu & -75.722590 & -4.536190 \\
\hline 339 & Loreto, Nauta, Pacaya & -73.609646 & -4.538165 \\
\hline 340 & Loreto, Parinari, Parinari, Amazon River & -74.433330 & -4.566660 \\
\hline 341 & Datem del Marañón, Pastaza, Ullpayacu, aprox. 5 km al NO de la boca del Río Pastaza & -76.598730 & -4.577180 \\
\hline 342 & Datem del Marañón, Manseriche, 4 km N Félix Flores & -77.498363 & -4.605397 \\
\hline 343 & Datem del Marañón, Pastaza, Trueno, aprox. 2 km al NO de la boca del Río Pastaza & -76.449880 & -4.647780 \\
\hline 344 & Loreto, Nauta, XX de Enero & -73.816667 & -4.650000 \\
\hline 345 & Loreto, Parinari, La Trocha & -74.420806 & -4.653583 \\
\hline 346 & Requena, Saquena, Río Ucayali, Sapwena (= Saquena) & -73.544688 & -4.661680 \\
\hline 347 & Loreto, Nauta, San Jacinto & -73.959394 & -4.673932 \\
\hline 348 & Loreto, Nauta, San Martín & -74.403028 & -4.688111 \\
\hline 349 & Loreto, Nauta, San Jacinto, $3 \mathrm{~km} \mathrm{~S}$ of & -73.959394 & -4.701459 \\
\hline 350 & Loreto, Nauta, Yarina & -73.983333 & -4.750000 \\
\hline 351 & Loreto, Parinari, Renacal & -74.516056 & -4.754083 \\
\hline 352 & Datem del Marañón, Barranca, Río Marañón, San Lorenzo & -76.600000 & -4.816667 \\
\hline 353 & $\begin{array}{l}\text { Datem del Marañón, Barranca, Nearly oposite mouth of Huallaga, Rio Marañon, San Lorenzo, Alto } \\
\text { Amazonas }\end{array}$ & -76.666667 & -4.833333 \\
\hline 354 & Requena, Jenaro Herrera, Jenaro Herrera, $1.4 \mathrm{~km}$ al N CIJH & -73.750000 & -4.904590 \\
\hline 355 & Requena, Jenaro Herrera, Jenaro Herrera & -73.672790 & -4.904720 \\
\hline 356 & Requena, Jenaro Herrera, Leuceo Herrera (= Jenaro Herrera) & -73.669774 & -4.907953 \\
\hline 357 & Requena, Jenaro Herrera, Centro de Investigaciones Jenaro Herrera & -73.750000 & -4.916667 \\
\hline 358 & Datem del Marañón, Manseriche, Comunidad Nativa Huáscar & -77.680544 & -4.956608 \\
\hline 359 & Loreto, Parinari, Yanayacu Grande/ La Huaca & -75.041111 & -4.973944 \\
\hline 360 & Datem del Marañón, Barranca, Pachacutec & -77.430556 & -4.993056 \\
\hline 361 & Loreto, Parinari, Río Samiria Estación Biológica de Pithecia & -74.540000 & -5.040000 \\
\hline 362 & Requena, Requena, Requena, Iglesia & -73.850030 & -5.058410 \\
\hline 363 & Loreto, Parinari, Pasto cocha & -75.100056 & -5.155944 \\
\hline 364 & Datem del Marañón, Barranca, Comunidad Nativa Wawajin & -77.430191 & -5.157111 \\
\hline 365 & Loreto, Parinari, Río Samiria, Base Atún/ Biological Station "Pithecia" & -74.666667 & -5.166667 \\
\hline 366 & Loreto, Parinari, Río Samiria, Santa Elena & -74.750000 & -5.183330 \\
\hline 367 & Requena, Yaquerana, Boca Río Yaquerana (= Alto Yavari Mirim, Boca Río Yaquerana) & -72.883333 & -5.200000 \\
\hline 368 & Loreto, Parinari, Pithecia & -74.669833 & -5.211306 \\
\hline 369 & Alto Amazonas, Lagunas , Lagunas & -75.633333 & -5.233300 \\
\hline 370 & Datem del Marañón, Barranca, Comunidad Nativa Nayumpin & -77.315863 & -5.234951 \\
\hline 371 & Datem del Marañón, Cahuapanas, Palmiche & -76.786333 & -5.240917 \\
\hline 372 & $\begin{array}{l}\text { Requena, Yaquerana, Matses Indian village of Nuevo San Juan }\left(5^{\circ} 14^{\prime} 50^{\prime \prime S}, 73^{\circ} 9^{\prime} 50^{\prime \prime} \mathrm{W} \text {, ca. } 150 \mathrm{~m} \text { above }\right. \\
\text { sea level) on the Río Gálvez, a left-bank tributary of the Río Yavarí }\end{array}$ & -73.163888 & -5.247222 \\
\hline 373 & Datem del Marañón, Cahuapanas, Cahuapanas & -76.983333 & -5.250000 \\
\hline 374 & Requena, Yaquerana, Nuevo San Juan (05¹5 S, $73^{\circ} 10$ W), Río Gálvez & -73.166667 & -5.250000 \\
\hline 375 & Datem del Marañón, Barranca, Belice & -77.541169 & -5.257927 \\
\hline 376 & Alto Amazonas, Jeberos, $4 \mathrm{~km}$ al SE de Jebedo & -76.275560 & -5.313330 \\
\hline 377 & Datem del Marañón, Barranca, Acho Norte & -77.475395 & -5.333899 \\
\hline 378 & Datem del Marañón, Cahuapanas, C.N. de Santa Martha & -77.213013 & -5.344217 \\
\hline 379 & Requena, Puinahua, Santa Cruz/ Campamento \#4 & -74.658972 & -5.485722 \\
\hline
\end{tabular}




\begin{tabular}{|c|c|c|c|}
\hline Pto. & Localidad & Grados X & Grados Y \\
\hline 380 & Requena, Emilio San Martín, Loreto, Yarinacocha (= Yarina Cocha) & -74.366667 & -5.516667 \\
\hline 381 & Requena, Puinahua, Alfaro & -75.047611 & -5.565444 \\
\hline 382 & Loreto, Parinari, El Caucho & -75.227889 & -5.617250 \\
\hline 383 & Datem del Marañón, Cahuapanas, Cerro Escalera, Alto Cahuapanas- Campamento 3 & -76.839167 & -5.664444 \\
\hline 384 & Requena, Tapiche, Iberia, río Tapiche & -74.150000 & -5.666667 \\
\hline 385 & Requena, Puinahua, Sitaraco & -75.119583 & -5.848972 \\
\hline 386 & Alto Amazonas, Balsapuerto, Cerro Escalera, Alto Cachiyacu - Intermedio & -76.760527 & -5.856111 \\
\hline 387 & Alto Amazonas, Balsapuerto, Cerro Escalera, Alto Cachiyacu-Campamento 2 & -76.717778 & -5.858611 \\
\hline 388 & Alto Amazonas, Balsapuerto, Cerro Escalera, Mina de sal-Trocha 1 & -76.604444 & -5.883889 \\
\hline 389 & Alto Amazonas, Yurimaguas, Pijuayo A & -76.107231 & -5.893599 \\
\hline 390 & Alto Amazonas, Yurimaguas, Yurimaguas & -76.083333 & -5.900000 \\
\hline 391 & Alto Amazonas, Yurimaguas, Pijuayo B & -76.133409 & -5.906485 \\
\hline 392 & Requena, Puinahua, Campamento \#6 & -75.183361 & -5.995278 \\
\hline 393 & Ucayali, Sarayacu, Río Ucayali & -74.967200 & -6.198330 \\
\hline 394 & Requena, Yaquerana, Cabeceras de Quebrada Sábalo, Zona Reservada Sierra del Divisor & -73.467361 & -6.365694 \\
\hline 395 & Requena, Soplín, Río Blanco & -73.720917 & -6.397111 \\
\hline 396 & Ucayali, Sarayacu, Maquia & -74.930270 & -6.406502 \\
\hline 397 & Requena, Soplín, Cabeceras de Quebrada Pantaleón, Zona Reservada Sierra del Divisor & -73.533028 & -6.423167 \\
\hline 398 & Requena, Yaquerana, Cabeceras de Quebrada Betilia, Zona Reservada Sierra del Divisor & -73.403333 & -6.434056 \\
\hline 399 & Requena, Yaquerana, Cabeceras de Quebrada Lobo, Zona Reservada Sierra del Divisor & -73.623917 & -6.507667 \\
\hline 400 & Requena, Alto Tapiche, Monte Alegre & -74.250000 & -6.700000 \\
\hline 401 & Ucayali, Vargas Guerra, Sarayacu, Ucayali River & -75.106111 & -6.817778 \\
\hline 402 & Ucayali, Vargas Guerra, Campamento El Fuerte, cerros de Orellana & -75.156910 & -6.908810 \\
\hline 403 & Ucayali, Inahuaya, Inahuaya & -75.283417 & -7.149972 \\
\hline 404 & Ucayali, Pampa Hermosa, Quebrada Panya, Topara River mouth & -75.233330 & -7.183330 \\
\hline 405 & Ucayali, Contamana, Sierra de Contamana, Cerros de Canchaguaya, Aguas Calientes & -74.948250 & -7.188920 \\
\hline 406 & Ucayali, Pampa Hermosa, Pampahermosa, Ucayali River, Cushavatay River & -75.266700 & -7.200000 \\
\hline 407 & Requena, Maquía, Sierra de Contamana, Cerros de Contaya, Alto Maquia A & -74.662330 & -7.238830 \\
\hline 408 & Requena, Maquía, Sierra de Contamana, Cerros de Contaya, Alto Maquia B & -74.666670 & -7.239830 \\
\hline 409 & Ucayali, Contamana, Contamana /Contamana Mts, El Indio & -74.900000 & -7.250000 \\
\hline 410 & Requena, Maquía, Sierra de Contamana, Cerros de Contaya B & -74.719830 & -7.252500 \\
\hline 411 & Requena, Maquía, Sierra de Contamana, Cerros de Contaya A & -74.696500 & -7.262000 \\
\hline 412 & Ucayali, Contamana, Sierra de Contamana & -74.791500 & -7.313670 \\
\hline 413 & Ucayali, Contamana, Cashiboya & -74.960398 & -7.318904 \\
\hline 414 & Ucayali, Contamana, Loreto, Contamana & -75.016667 & -7.333333 \\
\hline 415 & Ucayali, Pampa Hermosa, Pauya Campamento Ladera Camp 4 & -75.915139 & -7.560028 \\
\hline 416 & Ucayali, Contamana, Santa Rosa 1 & -74.840529 & -7.581466 \\
\hline 417 & Ucayali, Contamana, Santa Rosa 2 & -74.827487 & -7.612223 \\
\hline 418 & Ucayali, Contamana, Pisqui River, west tributary of Ucayali River & -75.050000 & -7.650000 \\
\hline 419 & Ucayali, Padre Márquez, San Pablo & -74.919756 & -7.726875 \\
\hline 420 & Ucayali, Padre Márquez, Quebrada Tunuya & -74.689130 & -7.776680 \\
\hline 421 & Ucayali, Padre Márquez, Roaboya, Ucayali River & -74.904400 & -7.793610 \\
\hline 422 & Ucayali, Padre Márquez, San Jeronimo & -74.844444 & -7.834167 \\
\hline 423 & Ucayali, Padre Márquez, San José de Vinuya & -74.712560 & -7.853260 \\
\hline
\end{tabular}




\begin{tabular}{|c|c|c|c|}
\hline Pto. & Localidad & Grados X & Grados Y \\
\hline 424 & Ucayali, Contamana, Pisqui Campamento Planicie y Pisqui trocha Huangana & -75.701444 & -8.404306 \\
\hline 425 & Ucayali, Contamana, Pisqui Campamento Playa y Pisqui Campamento Quebrada & -75.728666 & -8.432722 \\
\hline 426 & Mariscal Ramón Castilla, Yavarí, Quebrada Curacinha & -72.728333 & -5.051389 \\
\hline 427 & Mariscal Ramón Castilla, Yavarí, Quebrada Buenavista & -72.390278 & -4.834444 \\
\hline 428 & Mariscal Ramón Castilla, Yavarí, Quebrada Limera & -71.900833 & -4.514722 \\
\hline 429 & Maynas, Las Amazonas, Campamento Apayacu ( $\left.3^{\circ} 07^{\prime} 00^{\prime \prime S} 72^{\circ} 42^{\prime} 45^{\prime \prime} \mathrm{O}\right)$ & -72.712500 & -3.116667 \\
\hline 430 & Mariscal Ramón Castilla, Pebas, Campamento Maronal ( $\left.2^{\circ} 57^{\prime} 56.3^{\prime \prime S} 72^{\circ} 07^{\prime} 40.3^{\prime \prime O}\right)$ & -72.127861 & -2.965639 \\
\hline 431 & Putumayo, Yaguas, Campamento Yaguas ( $\left.2^{\circ} 51^{\prime} 53.5^{\prime \prime} \mathrm{S} 71^{\circ} 24^{\prime} 54.1^{\prime \prime} \mathrm{O}\right)$ & -71.415028 & -2.864861 \\
\hline 432 & Maynas, Torres Causana, Campamento Redondococha & -75.219222 & -0.571306 \\
\hline 433 & Maynas, Napo, Campamento Curupa & -73.018666 & -2.885028 \\
\hline 434 & Putumayo, Putumayo, Campamento Piedras & -72.917470 & -2.792750 \\
\hline 435 & Maynas, Mazán, Campamento Alto Mazán & -74.492500 & -2.586111 \\
\hline 436 & Maynas, Alto Nanay, Campamento Alto Nanay & -74.825277 & -2.806389 \\
\hline 437 & Maynas, Napo, Campamento Panguana & -75.149444 & -2.136944 \\
\hline 438 & Datem del Marañón, Morona, Pongo Chinim & -77.776222 & -3.113000 \\
\hline 439 & Datem del Marañón, Morona, Quebrada Wee & -77.529778 & -4.204111 \\
\hline 440 & Requena, Alto Tapiche, Ojo Contaya & -74.588500 & -7.115972 \\
\hline 441 & Requena, Alto Tapiche, Tapiche & -73.934472 & -7.208472 \\
\hline 442 & Requena, Alto Tapiche, Divisor & -73.882861 & -7.204556 \\
\hline 443 & Requena, Soplín, Wiswincho & -73.865550 & -5.810000 \\
\hline 444 & Requena, Soplín, Quebrada Pobreza & -73.773611 & -5.976667 \\
\hline 445 & Requena, Tapiche, Anguila & -73.910000 & -6.265000 \\
\hline
\end{tabular}

Anexo 2. Referencias que sustentan la presencia de cada especie en las localidades del Anexo 1.

Anoura aequatoris (Lönnberg, 1921)

Datem del Marañón.- 383: Patterson \& López Wong 2014.

Anoura caudifer (E. Geoffroy, 1818)

Datem del Marañón.- 353: Gardner 2008, Loreto.- 10: MUSM 41724, 11 : MUSM 41656-41657, 13: MUSM 41725, 14: KU 157993, 96: MUSM 26942. Maynas.- 3: MUSM 21000, 4: MUSM 20994-20999, 5: MUSM 20993, 7: MUSM 20992, 8: AMNH 71624, 41, 50: Wilson, Ascorra \& Solari 1996, 55: LSUMZ 28154-28159, 253: Díaz 2011, 316: Vásquez Újina 2019. Putumayo.- 30: Montenegro \& Moya 2011, 431: Montenegro \& Escobedo 2004 Montenegro \& Escobedo 2004. Requena.- 357: Wilson, Ascorra \& Solari 1996, 397: Medina et al. 2015, 398: Medina et al. 2015. Ucayali.- 402: MUSM 712-715, 405: MUSM 699, MUSM 700, MUSM 707.

Artibeus anderseni Osgood, 1916

Alto Amazonas.- 388: Patterson \& López Wong 2014, 391: MUSM 48005. Datem del Marañón.- 25: MUSM 39748, 45: MUSM 25812, 73: MUSM 25811, 256: FMNH 89049, FMNH 89050, FMNH 89135, 304: MUSM 1636116362, 341: MUSM 16363, 343: MUSM 16364, 360: MUSM 43044. Loreto. 11: MUSM 41660, 325: FMNH 122935-122938, MUSM 16365-16368, 344: Toffoli et al. 2018, 361: MUSM 1522-1525, 368: MUSM 47608. Mariscal Ramón Castilla.- 84: FMNH 89068, 327: FMNH 89058, FMNH 89136, 428: Escobedo 2003. Maynas.- 1: MUSM 21010, MUSM 21011, 8: Gardner 2008, 41: Wilson, Ascorra \& Solari 1996, 46: FMNH 87073, FMNH 89132, FMNH 89133, 50: Wilson, Ascorra \& Solari 1996, 51: LSUMZ 28408, 53: MUSM 42969, 57: MUSM 42970, 62: MUSM 32342, 63: LSUMZ 28436, 64: AMNH 98708, 66: AMNH 73480, 75: FMNH 87077-80779, FMNH 89134 77: MUSM 32317-32319, 80: MUSM 4359, MUSM 4360, 81: MUSM 28783, MUSM 28784, 83: MUSM 28785-28787, 87: TCWC 27445, TCWC 27448, TCWC 27449, 90: MUSM 28756, MUSM 28757, 139: MUSM 28817-28820, 142: MUSM 28752, 144: MUSM 28760, 147: MUSM 28807, 157: MUSM 32343, 158: MUSM 32324, MUSM 32325, 160: MUSM 28759, 171: MUSM
32359-32363, 173: MUSM 32364-32366, 178: MUSM 28767-28773, 179: MUSM 32383-32386, 180: Díaz 2011, 181: MUSM 32369-32382, 184: MUSM 28758, 188: MUSM 28821-28825, 189: MUSM 28753, MUSM 28754, 196: MUSM 28761-28766, 197: MUSM 32368, 198: TCWC 27446, 202: MUSM 28794, MUSM 28795, 206: MUSM 32357, MUSM 32358, 209: MUSM 28793, 210: MUSM 28796-28806, 211: MUSM 28810-28812, 220: MUSM 2881328816, 221: TCWC 29601, TCWC 29602, TCWC 29606, 223: MUSM 32367, 231: Wilson, Ascorra \& Solari 1996, 233: MUSM 28776-28778, 234: MUSM 28774, MUSM 28775, 235: MUSM 28779-28782, 237: MUSM 32333, 240: TCWC 27447, 243: MUSM 32327, 252: Hice et al. 2004, 255: MUSM 16477, MUSM 16478, 266: Díaz \& Linares García 2012, 270: MUSM 28788-28792, 271: MUSM 28755, 272: MUSM 32356, 276: MUSM 28808, MUSM 28809, 280: MUSM 32328-32330, 285: MUSM 32331, MUSM 32332, 288: MUSM 32344-32348, 292: MUSM 32354, 293: MUSM 32349-32353, 295: MUSM 32334-32341, 296: MUSM 32355, 305: MUSM 32326, 313: MUSM 32320, MUSM 32321, 318: MUSM 32322, MUSM 32323, 320: Ramos et al. 2017, 329: MUSM 21013, 335: MUSM 9425. Requena.- 355: Calderón \& Pacheco 2012, 357: Wilson, Ascorra \& Solari 1996, 367: FMNH 89057, 374: Voss et al. 2016, 379: MUSM 47607. Ucayali.- 401: AMNH 76256, 405: MUSM 17935, MUSM 17943, MUSM 17955, MUSM 18010, MUSM 18014, 412: Calderón \& Pacheco 2012.

Artibeus bogotensis Andersen, 1906

Requena.- 357: Ascorra et al. 1993

Artibeus cinereus (Gervais, 1856)

Maynas.- 252: Hice et al. 2004, 329: Wilson, Ascorra \& Solari 1996. Requena.- 395: Medina et al. 2015, 397-399: Medina et al. 2015.

Artibeus concolor Peters, 1865

Datem del Marañón.- 25: MUSM 39749. Maynas.- 127: Angulo \& Díaz 2004, 142: MUSM 28835, 160: MUSM 28836, 183: MUSM 32391: 212: MUSM 
45609, MUSM 45610, 214: MUSM 32393, 232: MUSM 32389, MUSM 32390, 244: MUSM 32388, 252: Hice et al. 2004, 259: MUSM 32387, 266: MUSM 28834, 322: MUSM 32392. Requena.- 357: Ascorra et al. 1993.

Artibeus glaucus Thomas, 1893

Datem del Marañón.- 341: Calderón \& Pacheco 2012. Loreto.- 208: MUSM 48233, 350: Toffoli et al. 2018. Maynas.- 80: MUSM 4210, 182: Bardales Ordoñez 2015, 316: Vásquez Újina 2019, 320: Ramos et al. 2017, 329: Wilson, Ascorra \& Solari 1996. Putumayo.- 30: Montenegro \& Moya 2011, 431: Montenegro \& Escobedo 2004. Requena.- 357: Ascorra et al. 1993, 374: Voss et al. 2016, 379: MUSM 47609, MUSM 47610, 398: Medina et al. 2015, 440: Jorge \& Velazco 2006. Ucayali.- 423: MUSM 38267.

\section{Artibeus gnomus Handley, 1987}

Alto Amazonas.- 388: Patterson \& López Wong 2014. Datem del Marañón.- 17: MUSM 41732, 37: MUSM 25813, MUSM 25814, 304: MUSM 16371, 341: MUSM 16372, MUSM 16373, 343: MUSM 16374. Loreto.- 14: KU 158000-158002, 68: MUSM 48234, 92: MUSM 39374, 347: KU 157995157999. Loreto.- 13: MUSM 41734, 48: MUSM 41733. Maynas.- 1: MUSM 21017-21024, 40: MUSM 21016, 52: MUSM 37613-37615, 59: MUSM 26579, 71: MUSM 27079, 173: MUSM 32442, 177: MUSM 28848, 179: MUSM 32443, 182: Bardales Ordoñez 2015, 187: MUSM 28852, MUSM 28853, 188: MUSM 28851, 190: MUSM 32440, MUSM 32441, 195: MUSM 28846, 204: MUSM 44899, MUSM 45633, 207: MUSM 44890-44894, 210: MUSM 28847, 211: MUSM 28849, 212: MUSM 44895-44898, 213: MUSM 28850, 218: MUSM 32431-32433, 226: MUSM 32428-32430, 230: MUSM 32434, MUSM 32435, 232: MUSM 32416, 235: MUSM 28845, 236: MUSM 3241032413, 242: MUSM 32436-32439, 247: MUSM 32427, 250: MUSM 28844, 252: Hice et al. 2004, 253: MUSM 28839-28843, 255: MUSM 16479-16482, 265: MUSM 28838, 266: MUSM 28837, 269: MUSM 32401, 278: MUSM 32402, 279: MUSM 32417, 281: MUSM 32403-32406, 282: MUSM 32408, 283: MUSM 32407, 285: MUSM 32409, 287: MUSM 32421, MUSM 32422 288: MUSM 32420, 293: MUSM 32423-32426, 295: MUSM 32414, MUSM 32415, 305: MUSM 32399, 310: MUSM 32394, MUSM 32395, 312: MUSM 32396, MUSM 32397, 316: Vásquez Újina 2019, 318: MUSM 32398, 320: Ramos et al. 2017, 328: MUSM 32400, 329: Wilson, Ascorra \& Solari 1996, 332: MUSM 32418, MUSM 32419. Requena.- 354: MUSM 1322, MUSM 1323, 357: Wilson, Ascorra \& Solari 1996, 374: Voss et al. 2016, 394, 395, 397-399: Medina et al. 2015.

Artibeus lituratus (Olfers, 1818)

Alto Amazonas.- 387, 388: Patterson \& López Wong 2014, 391: MUSM 48131-48134. Datem del Marañón.- 37: MUSM 25821, 45: MUSM 25824, MUSM 25825, 67: MUSM 25822, MUSM 25823, 72: MUSM 25826, 73: MUSM 25815-25820, 299: MUSM 37706, 301: MUSM 43046, 302: MUSM 43045, 304: MUSM 16338, 341: MUSM 16339, 360: MUSM 43047, 371: MUSM 43048, 383: Patterson \& López Wong 2014, 439: Castro Vergara 2012. Loreto.- 11: MUSM 41661, 18: MUSM 41619, 22: MUSM 41735, MUSM 41736, 325: FMNH 122871, KU 140230, KU 140234, KU 140260, 337: KU 140194, 347: KU 158003, KU 158004, 382: MUSM 47614. Mariscal Ramón Castilla.- 44: FMNH 104865, FMNH 104868, Maynas.- 1: MUSM 21027-21035, 3: MUSM 21026, 4: MUSM 21025, 41: Wilson, Ascorra \& Solari 1996, 46: FMNH 87015-87020, FMNH 87023-87027, FMNH 89153-89156, 50: Wilson, Ascorra \& Solari 1996, 55: LSUMZ 28217, LSUMZ 28233, LSUMZ 28241, LSUMZ 28243-28259, MUSM 1599-1606, MUSM 288, MUSM 289, 56: LSUMZ 28240, 63: LSUMZ 28424, LSUMZ 28425, 65: MUSM 37616, 75: FMNH 87030, FMNH 89157, 76: MUSM 32498, MUSM 32499, 77: MUSM 32445, MUSM 32446, 79: MUSM 37617, 81: MUSM 28941-28944, 83: MUSM 28945-28950, 90: MUSM 28882-28891, 93: MUSM 28977, MUSM 28978, 98: MUSM 32497, 106: FMNH 87010-87014, 110: MUSM 28909, 111: MUSM 28925, 114: AMNH 98701, 125: MUSM 28910, 126: MUSM 28868, 127: Angulo \& Díaz 2004, 131: MUSM 28867, 139: MUSM 28988, 142: MUSM 28873, MUSM 28874, 145: MUSM 28877- 28879, 146: MUSM 28875, MUSM 28876, 150: MUSM 32520, MUSM 32521, 151: MUSM 28912-28914, 158: MUSM 32470, MUSM 32471, 159: MUSM 29008, 160: MUSM 28905, 162: MUSM 28865, MUSM 28866, 164: MUSM 29003-29007, 166: MUSM 32522, MUSM 32523, 169: MUSM 28983, MUSM 28984, 171: MUSM 32518, MUSM 32519, 172: MUSM 32517, 180: MUSM 28995, MUSM 28996, 181: MUSM 32524, MUSM 32525, 182: Bardales Ordoñez 2015, 183: MUSM 32500, 188: MUSM 28989-28994, 189: MUSM 28880, MUSM 28881, 191: MUSM 2892628929, 195: MUSM 28934-28940, 196: MUSM 28911, 198: TCWC 27425, TCWC 27426, TCWC 27429, TCWC 27430, 199: MUSM 28930-28933, 202: MUSM 28963-28970, 204: MUSM 44860, MUSM 45615, 209: MUSM 28961, MUSM 28962, 210: MUSM 28971-28976, 211: MUSM 28985, 212: MUSM 44857-44859, MUSM 45611-45614, 213: MUSM 28986, MUSM 28987, 218: MUSM 32516, 221: TCWC 29585-29587, TCWC 29589, TCWC 29590, 226 :
MUSM 32515, 231: Wilson, Ascorra \& Solari 1996, 234: MUSM 28915, 236: MUSM 32493, 237: MUSM 32494-MUSM 32496, 238: MUSM 29009, 240: ROM 77385, ROM 77386, TCWC 27427, TCWC 27428, 241: MUSM 2901029015, 243: MUSM 32474, 244: MUSM 32480, 247: MUSM 32512-32514, 249: MUSM 32475-32479, 250: MUSM 28897-28904, 251: MUSM 28854, MUSM 28855, 252: Hice et al. 2004, Ramos-Rodríguez et al. 2018, 253: MUSM 28892-28896, 255: MUSM 16465, 257: MUSM 32444, 260: MUSM 28997-29002, 265: MUSM 28869-28872, 266: MUSM 28856-28864, 270: MUSM 28951-28960, 273: MUSM 28906-28908, 276: MUSM 28979-28982, 279: MUSM 32501, 280: MUSM 32486, 281: MUSM 32481-32483, 282: MUSM 32484, MUSM 32485, 285: MUSM 32487-32492, 286: MUSM 32473, 288: MUSM 32503-32508, 292: MUSM 32511, 293: MUSM 32509, MUSM 32510, 294: MUSM 32502, 305: MUSM 32472, 309: MUSM 32447, MUSM 32448, 312: MUSM 32449-32458, 314: MUSM 32459-32464, 316: Vásquez Újina 2019, 317: MUSM 26542, 318: MUSM 32465-32469, 320: Ramos et al. 2017, 329: Wilson, Ascorra \& Solari 1996, 335: MUSM 9422-9424, 429: Montenegro \& Escobedo 2004. Putumayo.- 23: Montenegro \& Moya 2011. Requena.- 355: MUSM 5929, 357: Wilson, Ascorra \& Solari 1996, 374: Voss et al. 2016, 395: Medina et al. 2015, 398: Medina et al. 2015, 399: Medina et al. 2015, 407: MUSM 20398, 440, 441: Jorge \& Velazco 2006. Ucayali.- 405: MUSM 17927, MUSM 17956, MUSM 17974, MUSM 18002, MUSM 18021, MUSM 18022, MUSM 18027, MUSM 18032, 414: Gardner 2008.

\section{Artibeus obscurus (Schinz, 1821)}

Alto Amazonas.- 376: MUSM 20967, 388: Patterson \& López Wong 2014. Datem del Marañón.- 16: MUSM 41662, MUSM 41663, 37: MUSM 25829, MUSM 25830, 67: MUSM 25831-25836, 73: MUSM 25827, MUSM 25828, 256: FMNH 89070-89073, 298: MUSM 43049, MUSM 43051, MUSM 43052, 302: MUSM 43050, 319: MUSM 20968, MUSM 20969, 343: MUSM 16340, MUSM 16341, 439: Castro Vergara 2012. Loreto.- 14: KU 158009-158015, 19: MUSM 41737, 22: MUSM 41738-41740, 29: MUSM 41620, 325: FMNH 122845-122849, FMNH 122864, KU 140261, 337: KU 140175, 338: MUSM 16342, 344: Toffoli et al. 2018, 347: KU 158005-158008, 350: Toffoli et al. 2018, 351: MUSM 47616, 361: MUSM 1571, MUSM 4756, 365: FMNH 122838-122844. Mariscal Ramón Castilla.- 331: FMNH 89074, 426, 427: Escobedo 2003, 430: Montenegro \& Escobedo 2004. Maynas.- 1: MUSM 21039-21059, 3: MUSM 21037, MUSM 21038, 4: MUSM 21036, 28: MUSM 24415, 32: LSUMZ 28429, 41: Wilson, Ascorra \& Solari 1996, 46: FMNH 87068, 50: Wilson, Ascorra \& Solari 1996, 55: LSUMZ 28189-28204, LSUMZ 28283, LSUMZ 28284, MUSM 1566-MUSM 1570, 59: MUSM 26543, 63: LSUMZ 28426-28428, 65: MUSM 37618, 71: MUSM 27080, 74: MUSM 37619, 76: MUSM 32594, MUSM 32595, 77: MUSM 32527, MUSM 32528, 79: MUSM 37620, MUSM 37621, 81: MUSM 29060, 110: MUSM 29036 MUSM 29037, 146: MUSM 29027, 164: MUSM 29125, 172: MUSM 32654, 180: MUSM 29112-29116, 181: MUSM 32660, 182: Bardales Ordoñez 2015, 187: MUSM 29117, MUSM 29118, 190: MUSM 32653, 195: MUSM 29059, 197: MUSM 32656-32659, 199: MUSM 29056-29058, 202: MUSM 29074 206: MUSM 32652, 209: MUSM 29071-29073, 210: MUSM 29075-29084, 212: MUSM 44861, MUSM 45616, 213: MUSM 29088-29090, 214: MUSM 32650, 217: MUSM 29091-29103, 218: MUSM 32649, 220: MUSM 2910429111, 221: TCWC 29579, TCWC 29580, TCWC 29582-29584, 223: MUSM 32655, 225: MUSM 42971, 231: Wilson, Ascorra \& Solari 1996, 233: MUSM 29040-29055, 234: MUSM 29038, MUSM 29039, 236: MUSM 32583-32585, 240: TCWC 27422-27424, 241: MUSM 29126- 29128, 242: MUSM 32651, 247: MUSM 32647, MUSM 32648, 251: MUSM 29016-29018, 252: Hice et al. 2004, 253: MUSM 29028-29032, 257: MUSM 32526, 260: Díaz 2011, 265: MUSM 29023-29026, 266: MUSM 29019-MUSM 29022, 269: MUSM 32563-32565, 270: MUSM 29061-29070, 273: MUSM 29033-29035, 274: MUSM 26580, 276: MUSM 29085-29087, 280: MUSM 32571-32576, 281: MUSM 32566, 282: MUSM 32569, MUSM 32570, 283: MUSM 32567, MUSM 32568, 285: MUSM 32577-32582, 287: MUSM 32610-32616, 288: MUSM 32604-32609, 290: MUSM 32603, 291: MUSM 32600-32602, 292: MUSM 32637-32639, MUSM 32643-32646, 293: MUSM 32617-32631, 294: MUSM 32596-32599, 295: MUSM 32586-32593, 296: MUSM 32632-32636, MUSM 32640-32642, 309: MUSM 32530-32535, 310: MUSM 32536-32540, 312: MUSM 32541-32555, 313: MUSM 32529, 314: MUSM 32556, MUSM 32557, 316: Vásquez Újina 2019, 317: MUSM 26544, 318: MUSM 32558-32562, 320: Ramos et al. 2017, 323: MUSM 32661, 329: Wilson, Ascorra \& Solari 1996, 335: MUSM 9429, MUSM 9430, 429: Montenegro \& Escobedo 2004, 432: Bravo \& Borman 2008, 433: Bravo 2010, 435, 436: Bravo \& Ríos 2007. Putumayo.- 36: MUSM 23167, 38: Montenegro \& Moya 2011. Requena.355: MUSM 865, MUSM 866, 357: Wilson, Ascorra \& Solari 1996, 374: Voss et al. 2016, 379: MUSM 47615, 394: Medina et al. 2015, 398, 399: Medina et al. 2015, 407: MUSM 20400, MUSM 20401, 408: MUSM 20402-20406, 411: MUSM 20399, 440-442: Jorge \& Velazco 2006. Ucayali.- 405: MUSM 17926, MUSM 17929, MUSM 17933, MUSM 17946, MUSM 17949, MUSM 
17950, MUSM 17984, MUSM 18000, MUSM 18006, MUSM 18011, MUSM 18016-18018, MUSM 18033, 412: MUSM 20407, MUSM 20408, 420: MUSM 38268-38274

Artibeus planirostris (Spix, 1823)

Alto Amazonas.- 388: Patterson \& López Wong 2014, 391: MUSM 4800648014, MUSM 48135-48155. Datem del Marañón.- 67: MUSM 25844-25848, 73: MUSM 25837-25843, 256: FMNH 89077, FMNH 89078, FMNH 89159 301: MUSM 43053-43063, 304: MUSM 16343, 341: MUSM 16344, 342 MUSM 38913, 360: MUSM 43064, MUSM 43065, 371: MUSM 43066-43068, 439: Castro Vergara 2012. Loreto.- 11: MUSM 41664, 14: KU 158017-158022, 21: MUSM 41741, 168: MUSM 41127, MUSM 41128, 208: MUSM 48235 325: FMNH 122861-122863, FMNH 122865-122870, KU 140222-140229, KU 140236-140247, KU 140250-140256, KU 140264, 337: KU 140184, KU 140191-140193, 338: MUSM 16337, 344: Toffoli et al. 2018, 347: KU 158016 350: Toffoli et al. 2018, 361: MUSM 1590-1595, MUSM 1629, MUSM 34103415, 365: FMNH 122850-122860, 368: MUSM 47612, MUSM 47613, 382 MUSM 47611. Mariscal Ramón Castilla.- 44: FMNH 104770, FMNH 104771, 84: FMNH 89075, FMNH 89076, 327: FMNH 89160, 426, 427, 428: Escobedo 2003. Maynas.- 1: MUSM 21069-21092, 3: MUSM 21060-21062, MUSM 21066-21068, 4: MUSM 21064, MUSM 21065, 5: MUSM 21063, 8: AMNH 71441, AMNH 71473, AMNH 71474, AMNH 71702-71707, AMNH 71711, 28: MUSM 24416, MUSM 24417, 41: Wilson, Ascorra \& Solari 1996, 46: FMNH 87021, FMNH 87022, 50: Wilson, Ascorra \& Solari 1996, 54: MUSM 42972, MUSM 42974, 55: LSUMZ 28205-28216, LSUMZ 28218-28232, LSUMZ 28234-28239, LSUMZ 28242, LSUMZ 28285, MUSM 1619-1628, 57: MUSM 42973, MUSM 42975, MUSM 42976, 58: MUSM 37625, MUSM 37626, 60: MUSM 37627, MUSM 37628, 65: MUSM 37622-37624, 74: MUSM 37630, 75: FMNH 87028, FMNH 87029, FMNH 89158, 76: MUSM 32732-32737, 80: MUSM 4350-4353, 81: MUSM 29368, MUSM 29369, 83: MUSM 29370 29375, 90: MUSM 29204-29218, 93: MUSM 29434, MUSM 29435, 94: MUSM 29436-29438, 95: MUSM 29157, 100: MUSM 29491-29497, 102: MUSM 32728, MUSM 32729-32731, 104: Díaz \& Linares García 2012, 106: FMNH 87009, 107: MUSM 29287, 108: MUSM 29282-29286, 110: MUSM 29274 29279, 111: MUSM 29345, 114: TCWC 29593, TCWC 29598, TCWC 29599, 115: MUSM 29170-29172, 125: MUSM 29280, MUSM 29281, 126: MUSM 29173-29175, 127: Angulo \& Díaz 2004, 129: MUSM 32740, 134: MUSM 29288, MUSM 29289, 135: MUSM 29158-29169, 139: MUSM 29487-29490, 142: MUSM 29179-29183, 143: MUSM 29428-29431, 144: MUSM 29255 MUSM 29256, 146: MUSM 29184-29198, 147: MUSM 29439-29444, 149: MUSM 32741, MUSM 32742, 151: MUSM 29300-29315, 152: MUSM 29199, MUSM 29200, 153: MUSM 29129-29137, 155: MUSM 32738, MUSM 32739 158: MUSM 32695, 159: MUSM 29543-29548, 160: Díaz \& Linares García 2012, 161: MUSM 37629, 162: MUSM 29155, MUSM 29156, 163: MUSM 29540, 164: MUSM 29541, MUSM 29542, 166: MUSM 32799, 169: MUSM 29455-29472, 171: MUSM 32796, 172: MUSM 32795, 173: MUSM 32797 MUSM 32798, 177: MUSM 29432, MUSM 29433, 178: MUSM 29293-29299, 179: MUSM 32807, 180: MUSM 29512-29517, 181: MUSM 32803-32806 182: Bardales Ordoñez 2015, 184: MUSM 29219-29221, 187: MUSM 29518 29534, 188: MUSM 29498-29511, 189: MUSM 29201-29203, 190: MUSM 32793, MUSM 32794, 191: MUSM 29346-29361, 195: MUSM 29367, 196: MUSM 29290-29292, 197: MUSM 32800-32802, 198: TCWC 27440-27444 199: MUSM 29362-29366, 202: MUSM 29402-29415, 204: MUSM 44864, MUSM 44865, 209: MUSM 29388-29401, 210: MUSM 29416, 29427, 212 MUSM 44862, MUSM 44863, MUSM 45617, 213: MUSM 29473-29475, 217: MUSM 29476-29478, 220: MUSM 29479-29486, 221: TCWC 29482 29490, TCWC 29588, TCWC 29592, TCWC 29594, TCWC 29596, TCWC 29600, 225: MUSM 42977-42980, 226: MUSM 32786, MUSM 32787, 227 MUSM 32789, 230: MUSM 32788, 233: MUSM 29325-29335, 234: MUSM 29316-29324, 235: MUSM 29336-29344, 236: MUSM 32724, 237: MUSM 32725-32727, 240: ROM 77383, ROM 77384, TCWC 27431-27439, TCWC 29597, 241: MUSM 29549-29555, 242: MUSM 32792, 244: MUSM 32703 32705, 247: MUSM 32784, MUSM 32785, 249: MUSM 32696-32702, 250 MUSM 29228-29239, 251: MUSM 29138-29142, 252: Hice et al. 2004, 253: MUSM 29222-29227, 260: MUSM 29535-29539, 265: MUSM 29176-29178, 266: MUSM 29143-29154, 269: MUSM 32706, 270: MUSM 29376-29387, 272: MUSM 32791, 273: MUSM 29257-29263, 275: MUSM 32790, 276: MUSM 29445-29454, 280: MUSM 32712-32716, 282: MUSM 32707-32711, 285: MUSM 32717-32723, 287: MUSM 32749-32753, 288: MUSM 32747 MUSM 32748, 292: MUSM 32775-32783, 293: MUSM 32754-32769, 294: MUSM 32744-32746, 296: MUSM 32770-32779, 309: MUSM 32662-32666 312: MUSM 32667-32682, 314: MUSM 32683-32687, 315: MUSM 32694 318: MUSM 32688-32693, 320: Ramos et al. 2017, 329: Wilson, Ascorra \& Solari 1996, 332: MUSM 32743, 335: MUSM 9426-9428. Putumayo.- 38: Montenegro \& Moya 2011, 431: Montenegro \& Escobedo 2004. Requena. 355: MUSM 5529, 357: Ascorra et al. 1993, 374: Voss et al. 2016, 394, 395:
Medina et al. 2015, 397-399: Medina et al. 2015, 407: MUSM 20415, 410: MUSM 20409, 411: MUSM 20410-20414, 440, 441: Jorge \& Velazco 2006 Ucayali.- 405: MUSM 17939, MUSM 17997, MUSM 18013, 415: Pacheco \& Arias 2001, 420: MUSM 38275

Carollia benkeithi Solari y Baker, 2006

Alto Amazonas.- 388: Patterson \& López Wong 2014. Datem del Marañón.- 342: MUSM 38916, MUSM 38917, 360: MUSM 43069. Loreto.- 347: KU 158034, KU 158035, 350: Toffoli et al. 2018, 361: MUSM 1144, MUSM 2917-2919, 365: FMNH 122836, 368: MUSM 47618, MUSM 47619, 382: MUSM 47617. Mariscal Ramón Castilla.- 426, 428: Escobedo 2003. Maynas.- 329: Wilson, Ascorra \& Solari 1996. Requena.- 355: Ruelas \& López 2018, 357: Ruelas \& López 2018, 374: Voss et al. 2016, 397, 399: Medina et al. 2015. Ucayali.- 402: MUSM 702, 405: MUSM 705, MUSM 706, 424: Pacheco \& Arias 2001.

Carollia brevicauda (Schinz, 1821)

Alto Amazonas.- 388: Patterson \& López Wong 2014, 391: MUSM 48015, MUSM 48016. Datem del Marañón.- 16: MUSM 41642, 72: MUSM 25849 MUSM 25850, 256: FMNH 89043-89046, 304: MUSM 16345, 319: MUSM 20970, 341: MUSM 16346, 342: MUSM 38914, MUSM 38915, 343: MUSM 16347, 358, 364, 370, 377, 378: Ruelas \& López 2018, 438, 439: Castro Vergara 2012. Loreto.- 11: MUSM 41639, 12: MUSM 41612, 14: KU 158027 158033, 24: MUSM 41605, MUSM 41606, MUSM 41610, 68: MUSM 48238 MUSM 48239, 208: MUSM 48236, MUSM 48237, 337: KU 140172, 338: MUSM 16348-16350, 344: Toffoli et al. 2018, 347: KU 158023-158026, 350: Toffoli et al. 2018, 361: MUSM 258, MUSM 3481, 365: FMNH 122835. Mariscal Ramón Castilla.- 430: Montenegro \& Escobedo 2004. Maynas.- 1 , 3, 4: Ruelas \& López 2018, 8: AMNH 71662-71664, 28: Ruelas \& López 2018, 41: Wilson, Ascorra \& Solari 1996, 49: MUSM 26522, 50: Wilson, Ascorra \& Solari 1996, 55: LSUMZ 28070, LSUMZ 28073, LSUMZ 28075, LSUMZ 28078, LSUMZ 28082, LSUMZ 28086-28088, LSUMZ 28090-28092, LSUMZ 28095, LSUMZ 28116, 58: MUSM 37632, 60: MUSM 37634, MUSM 37635 , 65: MUSM 37631, 76: MUSM 31065-31067, 77: MUSM 30977, 79: MUSM 37637, 80: MUSM 4335, 81: MUSM 27488-27492, 83: MUSM 27493, MUSM 27494, 85: MUSM 27532-27539, 90: MUSM 27415-27423, 114: TCWC 29609, TCWC 29610, 139: MUSM 27556, 140: MUSM 27405, 143: MUSM 27531 146: MUSM 27411, MUSM 27412, 153: MUSM 27385, MUSM 27386, 158: MUSM 31021, 160: MUSM 27442-27445, 161: MUSM 37636, 167: MUSM 31064, 169: MUSM 27543, 171: MUSM 31127-31139, 172: MUSM 31126, 173: MUSM 31140, MUSM 31141, 175: MUSM 27588-27590, 178: Díaz \& Linares García 2012, 179: MUSM 31160-31164, 180: MUSM 27565-27568, 181: MUSM 31158, MUSM 31159, 182: Bardales Ordoñez 2015, 184: MUSM 27424, 187: MUSM 27569-27579, 188: MUSM 27557-27564, 189: MUSM 27413, MUSM 27414, 191: MUSM 27472-27476, 192: MUSM 27487, 195: MUSM 27482-27486, 196: MUSM 27452-27454, 197: MUSM 31154-31157, 199: MUSM 27477-27481, 201: MUSM 37633, 202: MUSM 27516-27520, 204: MUSM 44870, MUSM 44871, 205: MUSM 31022, 207: MUSM 44866, 209: MUSM 27509-27515, 210: MUSM 27521-27530, 212: MUSM 4486744869, MUSM 45618-45621, 213: MUSM 27544-27548, 214: MUSM 31123 217: MUSM 27549-27552, 218: MUSM 31120, 220: MUSM 27553-27555, 221: TCWC 29607, TCWC 29608, 223: MUSM 31153, 226: MUSM 31119, 230: MUSM 31121, MUSM 31122, 231: Wilson, Ascorra \& Solari 1996, 232: MUSM 31068, MUSM 31069, 233: MUSM 27467-27471, 234: MUSM 27465, MUSM 27466, 236: MUSM 31057-31061, 238: MUSM 27586, 241: MUSM 27587, 242: MUSM 31124, MUSM 31125, 243: MUSM 31029, 244: MUSM 31039, MUSM 31040, 247: MUSM 31116-MUSM 31118, 249: MUSM 31030-31038, 250: MUSM 27436-27441, 251: Díaz \& Linares García 2012, 252: Hice et al. 2004, 253: MUSM 27425-27435, 255: Ruelas \& López 2018, 259: MUSM 31027, 260: Díaz 2011, 261: MUSM 31145-31152, 264: MUSM 31142-MUSM 31144, 265: Díaz 2011, 266: MUSM 27398-27404, 269: MUSM 31041, MUSM 31042, 270: Díaz \& Linares García 2012, 273: MUSM 27446-27451, 274: MUSM 26568, 276: Díaz \& Linares García 2012, 277: MUSM 31070, 278: MUSM 31043, 279: MUSM 31071, 280: MUSM 3105231054, 281: MUSM 31044-31047, 282: MUSM 31051, 283: MUSM 31048, 284: MUSM 31049, MUSM 31050, 285: MUSM 31055, MUSM 31056, 286: MUSM 31028, 287: MUSM 31093-31097, 288: MUSM 31086-31092, 290: MUSM 31083-31085, 292: MUSM 31106-31108, MUSM 31112-31115, 293: MUSM 31098-31104, 294: MUSM 31079-31082, 295: MUSM 31062, MUSM 31063, 296: MUSM 31105, MUSM 31109-31111, 300: MUSM 31072-31075, 308: MUSM 31023, MUSM 31024, 309: MUSM 30981-30987, 310: MUSM 30988-30990, 312: Ruelas \& López 2018, 313: MUSM 30978-30980, 314: MUSM 31004-31009, 315: MUSM 31014-31020, 316: Vásquez Újina 2019 317: MUSM 26523, MUSM 26524, 318: MUSM 31010-31013, 320: Ramos et al. 2017, 323: MUSM 31165-31167, 328: MUSM 31025, MUSM 31026, 329: Wilson, Ascorra \& Solari 1996, 332: MUSM 31076-31078, 335: MUSM 
9417, 436, 437: Bravo \& Ríos 2007. Putumayo.- 15: Montenegro \& Moya 2011. Requena.- 355: Ruelas \& López 2018, 357: Wilson, Ascorra \& Solari 1996, 374: Voss et al. 2016, 394, 395, 397-399: Medina et al. 2015, 407: MUSM 20416-20418, 440-442: Jorge \& Velazco 2006. Ucayali.- 396: Ruelas \& López 2018, 405: MUSM 17948, MUSM 17957, MUSM 18008, MUSM 5576, 412: MUSM 20419, MUSM 20420, 413: Ruelas \& López 2018, 415: Pacheco \& Arias 2001, 416, 417, 419: Ruelas \& López 2018, 420: MUSM 38276, MUSM 38277

\section{Carollia perspicillata (Linnaeus, 1758)}

Alto Amazonas.- 388: Patterson \& López Wong 2014, 391: MUSM 4815648158. Datem del Marañón.- 16: MUSM 41643, 17: MUSM 41687-41693, 25: MUSM 39750, 37: MUSM 25853, MUSM 25854, 45: MUSM 25859, 67: MUSM 25855-25858, 72: MUSM 25860, MUSM 25861, 298: MUSM 43070, MUSM 43071, 336: MUSM 38922, 341: MUSM 16354, 342: MUSM 38918-38921, 343: Ruelas \& López 2018, 52: USNM 240288, 360: MUSM 43072-43077, 371: MUSM 43078-43080, 375: Ruelas \& López 2018, 438 Castro Vergara 2012. Loreto.- 10: MUSM 41699-41708, 11: MUSM 4164441649, 12: MUSM 41611, MUSM 41613, 13: MUSM 41711, MUSM 41712, 14: KU 158042-158045, 19: MUSM 41694, 20: MUSM 41614, 22: MUSM 41695-41698, 24: MUSM 41609, 27: MUSM 41709, MUSM 41710, 68: MUSM 48248, 69: MUSM 48240, MUSM 48241, 168: MUSM 41129, 208: MUSM 48242-48247, 339: Ruelas \& López 2018, 344: Toffoli et al. 2018, 347: KU 158038-158041, 350: Toffoli et al. 2018, 359: MUSM 38386, MUSM 47621, 361: MUSM 1415, MUSM 3565, 365: FMNH 122833, FMNH 122834. Mariscal Ramón Castilla.- 327: FMNH 89047, FMNH 89048, 426, 427, 428: Escobedo 2003, 430: Montenegro \& Escobedo 2004. Maynas.- 1: Ruelas \& López 2018, 3: MUSM 21116, MUSM 21117, 4: MUSM 21111-21115, 7: Ruelas \& López 2018, 8: AMNH 71427-71440, AMNH 71442-71453, 28: MUSM 24419, 32: LSUMZ 28430, LSUMZ 28431, 41, 50: Wilson, Ascorra \& Solari 1996, 52: MUSM 37642, 53: MUSM 42982, 54: MUSM 42983, 55: LSUMZ 28068-28072, LSUMZ 28074, LSUMZ 28076-28081, LSUMZ 28083-28085, LSUMZ 28093, LSUMZ 28094, LSUMZ 28096, LSUMZ 28097, 56: LSUMZ 28065-28067, 57: MUSM 42981, 58: MUSM 37643-37646, MUSM 37652, 60: MUSM 3764737650, 64: AMNH 98704-98706, AMNH 98772, 66: AMNH 73467, AMNH 73476, AMNH 73482-73494, AMNH 74006, AMNH 74007, 70: AMNH 7399474005, 74: MUSM 37641, 76: MUSM 31429-MUSM 31431, 77: MUSM 31265, MUSM 31266, 79: MUSM 37651, 80: MUSM 4207-4209, MUSM 4214, 81: MUSM 27683, MUSM 27925-27929, 83: MUSM 27930-27933, 85: Díaz \& Linares García 2012, 86: AMNH 130178-130185, 87: TCWC 27450-27454, 90: MUSM 27712-27728, 93: MUSM 28029, 98: MUSM 31418-31426, 106 FMNH 87046-87054, 110: MUSM 27793, MUSM 27794, 111: MUSM 27894, MUSM 27895, 122: MUSM 31427, MUSM 31428, 129: MUSM 31434, MUSM 31435, 134: MUSM 27795, 139: MUSM 28101-28105, 140: MUSM 2768427687, 142: MUSM 27702, 143: MUSM 28024-28026, 144: MUSM 2777027776, 146: MUSM 27703-27705, 151: MUSM 27818-27830, 152: MUSM 27706, MUSM 27707, 153: MUSM 27641, MUSM 27642, 157: MUSM 31432, MUSM 31433, 159: MUSM 28198, MUSM 28199, 160: MUSM 27766-27769, 163: MUSM 28185-28187, 164: MUSM 28188-28197, 166: MUSM 3154931551, 169: MUSM 28048-28066, 171: MUSM 31533-31545, 172: MUSM 31532, 173: MUSM 31546-31548, 175: MUSM 28232-28243, 177: MUSM 28027, MUSM 28028, 178: MUSM 27811- 27817, 179: MUSM 31577-31580, 180: Díaz 2011, 181: MUSM 31573-31576, 182: Bardales Ordoñez 2015, 183: MUSM 31437, MUSM 31438, 184: MUSM 27729-27731, 186: MUSM 31262-31264, 187: MUSM 28158-28163, 188: MUSM 28106-28129, 189: MUSM 27708-27710, 190: MUSM 31531, 191: MUSM 27896-27906, 192, 195: Díaz \& Linares García 2012, 196: MUSM 27796-27810, 199: MUSM 27907- 27913, 200: MUSM 31530, 202: MUSM 27978-28002, 204: MUSM 44880-44887, MUSM 45626-45632, 205: MUSM 31342-31350, 207: MUSM 44875-44877, 209: MUSM 27958-27977, 210: MUSM 28003-28023, 211: MUSM 28067-28070, 212: MUSM 44878, MUSM 44879, MUSM 4562345625, 213: MUSM 28071-28081, 214: MUSM 31525-31528, 215: Ruelas \& López 2018, 217: MUSM 28082-28087, 218: Ruelas \& López 2018, 220: MUSM 28088-28100, 223: MUSM 31570-31572, 224: Ruelas \& López 2018, 225: MUSM 42984-42986, 228: MUSM 31519, 229: MUSM 31521, MUSM 31522, 230: MUSM 31520, 231: Wilson, Ascorra \& Solari 1996, 232: MUSM 31355, MUSM 31356, MUSM 31436, 233: MUSM 27855-27874, 234: MUSM 27831-27854, 235: MUSM 27875-27893, 236: MUSM 31404-31414, 237: MUSM 31415, MUSM 31416, 238: Díaz \& Linares García 2012, 239: MUSM 31357, MUSM 31358, 241: MUSM 28219-28231, 244: MUSM 31364-MUSM 31366, 245: MUSM 31359, 247: MUSM 31508-31510, 248: Ruelas \& López 2018, 249: MUSM 31360-31363, 250: MUSM 27749-27765, 251: MUSM 27643-27656, 252: Hice et al. 2004, 253: MUSM 27732-27748, 255: Ruelas \& López 2018, 257: MUSM 31261, 260: Díaz 2011, 261: MUSM 31560-31569, 263: MUSM 31557, MUSM 31558, 264: MUSM 31552-31556, 265: MUSM 27688-27701, 266: Díaz \& Linares García 2012, 267: MUSM 31559, 270:
MUSM 27934-27957, 271: MUSM 27711, 272: MUSM 31529, 273: MUSM 27777-27792, 274: MUSM 26567, 276: MUSM 28031-28047, 277: MUSM 31439, 279: MUSM 31440, 280: MUSM 31382-31390, 281: MUSM 31367, MUSM 31368, 282: MUSM 31374-31381, 283: MUSM 31369, 284: MUSM 31370-31373, 285: Ruelas \& López 2018, 286: MUSM 31354, 287: MUSM 31468-31471, 288: MUSM 31448-31467, 290: MUSM 31447, 291: MUSM 31446, 292: MUSM 31502-31507, 293: MUSM 31472-31494, 294: MUSM 31443-31445, 295: MUSM 31417, 296: MUSM 31495-31501, 300: MUSM 31441, MUSM 31442, 303: MUSM 31334, MUSM 31335, 306: MUSM 31351, 309: MUSM 31283-31289, 310: MUSM 31290-31295, 312: MUSM 31296-31303, MUSM 31305-31313, 313: Ruelas \& López 2018, 314: MUSM 31314-31331, 315: MUSM 31336-31341, 316: Vásquez Újina 2019, 318: MUSM 31332, MUSM 31333, 320: Ramos et al. 2017, 323: MUSM 31581, MUSM 31582, 328: MUSM 31352, MUSM 31353, 329: Wilson, Ascorra \& Solari 1996, 335: Ruelas \& López 2018, 429: Montenegro \& Escobedo 2004, 433: Bravo 2010, 436, 437: Bravo \& Ríos 2007. Putumayo.- 6: AMNH 98774, AMNH 98776, AMNH 98778, 15, 23, 30: Montenegro \& Moya 2011, 431: Montenegro \& Escobedo 2004. Requena.- 355: Ruelas \& López 2018, 357: Wilson, Ascorra \& Solari 1996, 374: Voss et al. 2016, 379: MUSM 47620, 398: Medina et al. 2015, 399: Medina et al. 2015, 400: AMNH 67226, 407: MUSM 20421, 440, 442: Jorge \& Velazco 2006, 445: Escobedo 2015. Ucayali.- 396: Ruelas \& López 2018, 401: AMNH 76219-76235, 405: Ruelas \& López 2018, 412: MUSM 20422-20424, 413, 416, 417, 419: Ruelas \& López 2018, 420: MUSM 38278-38282, 423: MUSM 38283, MUSM 38284.

Carollia sp. (sensu Solari \& Baker 2006)

Datem del Marañón.- 37: MUSM 25851, MUSM 25852, 256: FMNH 8910789109, 343: MUSM 16351. Loreto.- 10: MUSM 41679-41685, 11: MUSM 41640, MUSM 41641, 13: MUSM 41686, 14: KU 158036, KU 158037, 22: MUSM 41678, 24: MUSM 41607, 29: MUSM 41608, 48: MUSM 41677, 338: MUSM 16352, MUSM 16353. Mariscal Ramón Castilla.- 430: Montenegro \& Escobedo 2004. Maynas.- 3: MUSM 21132, MUSM 21133, 4: MUSM 21129-21131, 5: MUSM 21127, MUSM 21128, 8: AMNH 71632, AMNH 71633, 41, 50: Wilson, Ascorra \& Solari 1996, 51: LSUMZ 28409, 55: LSUMZ 28089, LSUMZ 28098-28109, LSUMZ 28111-28115, LSUMZ 28117-28142, LSUMZ 28144-28152, MUSM 1437-1446, KU 143591, 62: MUSM 31215, MUSM 31216, 66: Pirlot 1968, 74: MUSM 37638, 76: MUSM 31217-31225, 79: MUSM 37640, 81: MUSM 27611, 90: MUSM 27593, MUSM 27594, 173: MUSM 31253, MUSM 31254, 178: MUSM 27599, 179: MUSM 31259, 180: MUSM 27634, MUSM 27635, 181: MUSM 31257, MUSM 31258, 182: Bardales Ordoñez 2015, 188: MUSM 27633, 191: MUSM 27608, 195: MUSM 27610, 197: MUSM 31256, 199: MUSM 27609, 201: MUSM 37639, 202: MUSM 27621-27623, 204: MUSM 44872-44874, MUSM 45622, 209: Ruelas \& López 2018, 210: MUSM 27624-27627, 213: Ruelas \& López 2018, 217: MUSM 27631, 220: MUSM 27632, 230: MUSM 31251, 233: MUSM 2760227607, 234: MUSM 27600, MUSM 27601, 236: MUSM 31197-31204, 241: MUSM 27640, 247, 248: Ruelas \& López 2018, 252: Hice et al. 2004, 253: MUSM 27595, MUSM 27596, 260, 265: Ruelas \& López 2018, 267: MUSM 31255, 270: Ruelas \& López 2018, 272: MUSM 31252, 273: Ruelas \& López 2018, 276: MUSM 27628, 277: MUSM 31226, 279: MUSM 31227-31229, 280: MUSM 31192, MUSM 31193, 281: MUSM 31188, MUSM 31189, 282: MUSM 31190, MUSM 31191, 285: MUSM 31194-31196, 286: MUSM 31187, 287: MUSM 31237, MUSM 31238, 288: MUSM 31235, MUSM 31236, 292: MUSM 31245, MUSM 31247, 293: MUSM 31239-31243, 294: MUSM 31231-31234, 295: MUSM 31205-31214, 296: MUSM 31244, MUSM 31246, 305: MUSM 31184, 309: MUSM 31169, 310: MUSM 31170-31172, 312, 313: Ruelas \& López 2018, 314: MUSM 31180-31182, 318: MUSM 31183, 320: Ramos et al. 2017, 322: MUSM 31230, 323: MUSM 31260, 328: MUSM 31185, MUSM 31186, 335: Ruelas \& López 2018, 429: Montenegro \& Escobedo 2004. Putumayo.- 15, 30, 38: Montenegro \& Moya 2011, 431: Montenegro \& Escobedo 2004. Requena.- 357: Wilson, Ascorra \& Solari 1996, 374: MUSM 15165.

Centronycteris maximiliani (J. Fischer, 1829)

Maynas.- 252: Hice et al. 2004, 255: Hice \& Solari 2002, 277: MUSM 30846, 332: MUSM 30847, Requena.- 394: Medina et al. 2015.

Chiroderma salvini Dobson, 1878

Mariscal Ramón Castilla.- 426, 428: Escobedo 2003. Maynas.- 77: MUSM 32809, 179: MUSM 32814, 230: MUSM 32813, 252: Hice et al. 2004, 285: MUSM 32810, 295: MUSM 32811, MUSM 32812, Requena.- 398: Medina et al. 2015.

Chiroderma trinitatum Goodwin, 1958

Datem del Marañón.- 256: FMNH 89092. Loreto.- 347: KU 158046, 368: MUSM 38388, MUSM 47627. Loreto.- 22: MUSM 41742. Mariscal Ramón Castilla.- 327: FMNH 89083, FMNH 89085, FMNH 89093. Maynas.- 40: 
MUSM 21134, MUSM 21135, 41: Wilson, Ascorra \& Solari 1996, 83: MUSM 29556, 179: MUSM 32824, 198: TCWC 27455, 202: MUSM 29557, 207: MUSM 44888, MUSM 44889, 218: MUSM 32823, 226: MUSM 32822, 232: MUSM 32818, 240: TCWC 27456-27460, 241: MUSM 29559, 243: MUSM 32819, 249: MUSM 32820, 252: Hice et al. 2004, 260: MUSM 29558, 282: MUSM 32821, 310: MUSM 32815, 311: MUSM 32817, 312: MUSM 32816. Requena.- 357: Wilson, Ascorra \& Solari 1996, 395: Medina et al. 2015, 398, 399: Medina et al. 2015, 441: Jorge \& Velazco 2006. Ucayali.- 405: MUSM 17945.

\section{Chiroderma villosum Peters, 1860}

Datem del Marañón.- 298: MUSM 43081, 342: MUSM 38923, 352: 0 Thomas 1927a. Loreto.- 208: MUSM 48249, 325: FMNH 122932- 122934 337: KU 140197-140199, 347: KU 158047, 361: MUSM 1637-1639, MUSM 2940, 365: FMNH 122919- 122931, 382: MUSM 47628. Mariscal Ramón Castilla.- 44: FMNH 104866, 327: FMNH 89079, FMNH 89080. Maynas.1: MUSM 21137, MUSM 21138, 3: MUSM 21136, 41: Wilson, Ascorra \& Solari 1996, 46: FMNH 87031- 87034, FMNH 87045, 50: Wilson, Ascorra \& Solari 1996, 55: LSUMZ 28263, LSUMZ 28277, 59: MUSM 26545, 75: FMNH 87035, 176: USNM 337940, 188: MUSM 29563, 191: MUSM 29560, 195: MUSM 29561, 198: TCWC 27461, TCWC 27462, 226: MUSM 32832, 240: TCWC 27463-27467, 252: Hice et al. 2004, 255: MUSM 16476, 258: MUSM 32829, 270: MUSM 29562, 280: MUSM 32827, 285: MUSM 32828, 288 MUSM 32830, 293: MUSM 32831, 309: MUSM 32825, 312: MUSM 32826, 316: Vásquez Újina 2019, 320: Ramos et al. 2017. Requena.- 357: Wilson, Ascorra \& Solari 1996, 395, 399: Medina et al. 2015. Ucayali.- 415, 424: Rodríguez et. al. 2001.

\section{Choeroniscus minor (Peters, 1868)}

Datem del Marañón.- 304: MUSM 16358, 333: AMNH 98734, 343: MUSM 16359, MUSM 16360, 438: Castro Vergara 2012. Loreto.- 14: KU 158049, KU 158050, 27: MUSM 41726, 347: KU 158048, 361: MUSM 4757. Maynas.- 1: MUSM 21139-21141, 51: LSUMZ 28410, 74: MUSM 37653, 218: MUSM 31812, 252: Hice et al. 2004, 272: MUSM 31813, 277: MUSM 31807, 286: MUSM 31806, 294: MUSM 31810, 296: MUSM 31811, 307: MUSM 31803, MUSM 31804, 312: MUSM 31802, 320: Ramos et al. 2017, 322: MUSM 31808, MUSM 31809, 328: MUSM 31805. Requena.- 355: MUSM 862, 357: Wilson, Ascorra \& Solari 1996, 374: Voss et al. 2016, 399: Medina et al. 2015.

\section{Chrotopterus auritus (Peters, 1856)}

Maynas.- 1: MUSM 21143, MUSM 21144, 40: MUSM 21142, 41: Wilson, Ascorra \& Solari 1996, 182: Bardales Ordoñez 2015, 186: MUSM 31975, MUSM 31976, 190: MUSM 31982, 218: MUSM 31980, MUSM 31981, 251: MUSM 28571, 252: Hice et al. 2004, 268: MUSM 31983, 277: MUSM 31979, 286: MUSM 31978, 311: MUSM 31977. Requena.- 354: Ascorra et al. 1993, 357: Wilson, Ascorra \& Solari 1996, 374: Voss et al. 2016, 442: Jorge \& Velazco 2006

\section{Cormura brevirostris (Wagner, 1843)}

Datem del Marañón.- 438: Castro Vergara 2012. Mariscal Ramón Castilla. 289: FMNH 89169, 327: FMNH 89122-89124. Maynas.- 8: AMNH 71637, 46: FMNH 87108-87111, 66: AMNH 73501, 70: AMNH 74103, AMNH 74104, AMNH 74106, 202: Díaz \& Linares García 2012, 233: MUSM 27142, 252: Hice et al. 2004, 253: Díaz \& Linares García 2012, 259: MUSM 30849, 293: MUSM 30851, 309: MUSM 30848, 332: MUSM 30850. Requena.- 355: Ascorra et al. 1993, 374: Voss et al. 2016, 395: Medina et al. 2015.

Cynomops milleri (Osgood, 1914)

Alto Amazonas.- 390: FMNH 19652 (Pacheco et al. 2009).

Cynomops planirostris (Peters, 1866)

Maynas.- 100: Díaz 2011, 249: MUSM 30918.

Cyttarops alecto Thomas 1913

Requena.- 394: Ludeña \& Medina 2017.

Desmodus rotundus (E. Geoffroy, 1810)

Alto Amazonas.- 388: Patterson \& López Wong 2014. Datem del Marañón.- 298: MUSM 43114-43126, 301: MUSM 43127-43135, 343: Quintana \& Pacheco 2007, 360: MUSM 43082-43093, 371: MUSM 43094-43113, MUSM 43136. Loreto.- 14: Quintana \& Pacheco 2007, 22: MUSM 41723, 325: Quintana \& Pacheco 2007, 338, 345, 348: Quintana \& Pacheco 2007. Maynas.- 1, 3, 8, 40: Quintana \& Pacheco 2007, 41: Wilson, Ascorra \& Solari 1996, 53: MUSM 42989, MUSM 42995, MUSM 42996, 54: MUSM 42987, MUSM 42988, MUSM 42990-42993, MUSM 42997-42999, 57: MUSM 42994, 77, 169, 178: Quintana \& Pacheco 2007, 182: Bardales Ordoñez 2015, 183,
186, 188, 210: Quintana \& Pacheco 2007, 225: MUSM 43000-43003, 231: Wilson, Ascorra \& Solari 1996, 232: Quintana \& Pacheco 2007, 244, 247 Quintana \& Pacheco 2007, 252: Hice et al. 2004, 261, 277, 294, 296, 300, 310-312: Quintana \& Pacheco 2007, 316: Vásquez Újina 2019, 320: Ramos et al. 2017, 323: Quintana \& Pacheco 2007. Requena.- 355: Quintana \& Pacheco 2007, 357: Wilson, Ascorra \& Solari 1996, 374: Quintana \& Pacheco 2007.

Diaemus youngi (Jentink, 1893)

Datem del Marañón.- 298: MUSM 43139, 301: MUSM 43140, MUSM 43141 360: MUSM 43137, MUSM 43138. Mariscal Ramón Castilla.- 47: Gardner 2008. Maynas.- 57: MUSM 43004.

Diclidurus albus Wied-Neuwied, 1820

Loreto.- 340: Escobedo \& Velazco 2012

Diclidurus isabella (Thomas, 1920)

Mariscal Ramón Castilla.- 334: Escobedo \& Velazco 2012.

Diphylla ecaudata Spix, 1823

Datem del Marañón.- 256: Quintana \& Pacheco 2007, 298: MUSM 43142, MUSM 43144, 302: MUSM 43143, 360: MUSM 43145-43155, 371: MUSM 43156, MUSM 43157. Loreto.- 14: Quintana \& Pacheco 2007. Maynas.- 316: Vásquez Újina 2019, 437: Bravo \& Ríos 2007. Requena.- 374: Quintana \& Pacheco 2007, 397-399: Medina et al. 2015. Ucayali.- 424: Pacheco \& Arias 2001.

Enchisthenes hartii (Thomas, 1892)

Mariscal Ramón Castilla.- 427, 428: Escobedo 2003. Requena.- 357: Wilson Ascorra \& Solari 1996, 440: Jorge \& Velazco 2006. Ucayali.- 425: Pacheco \& Arias 2001

Eptesicus brasiliensis (Desmarest, 1819)

Datem del Marañón.- 360: MUSM 43169. Loreto.- 14: KU 158157-158159. Maynas.- 66: AMNH 74016, 100: MUSM 30024, 122: MUSM 33403, 144: MUSM 30023, 149: MUSM 33404, 194: MUSM 33406, 252: Hice et al. 2004 296: MUSM 33405. Requena.- 357: Wilson, Ascorra \& Solari 1996, 394: Medina et al. 2015, 400: AMNH 67232, AMNH 67233.

Eptesicus furinalis (d'Orbigny, 1847)

Maynas.- 163: MUSM 30026, 251: MUSM 30025.

Eumops delticus Thomas, 1923

Maynas.- 165: Díaz 2011.

Eumops hansae Sanborn, 1932

Maynas.- 207: MUSM 44836, 329: Wilson, Ascorra \& Solari 1996. Requena.398: Medina et al. 2015, 399: Medina et al. 2015.

Eumops maurus (Thomas, 1901)

Maynas.- 140: Díaz 2011.

Eumops trumbulli (Thomas, 1901)

Mariscal Ramón Castilla.- 84: Medina et al. 2014. Maynas.- 43: Koopman 1971.

Furipterus horrens (F. Cuvier, 1828)

Maynas.- 63: Gardner 2008, 70: AMNH 74108. Requena.- 357: Wilson, Ascorra \& Solari 1996, 374: Voss et al. 2016. Ucayali.- 418: AMNH 98731.

Gardnerycteris crenulatum (E. Geoffroy, 1803)

Alto Amazonas.- 386: Patterson \& López Wong 2014. Datem del Marañón.- 341: MUSM 16382. Loreto.- 14: KU 158070, KU 158071. Mariscal Ramón Castilla.- 331: FMNH 89038-89040, FMNH 89151, FMNH 89152. Maynas.- 1: MUSM 21200, 8: AMNH 71690, 31: MUSM 24423, 34: LSUMZ 34956, 40: MUSM 21199, 41, 50: Wilson, Ascorra \& Solari 1996, 62: MUSM 32080, MUSM 32081, 76: MUSM 32082, 77: MUSM 32052-32055, 81: MUSM 28602, 90: MUSM 28593, 150: MUSM 32102, 169: MUSM 28619, 171: MUSM 32098-32100, 173: MUSM 32101, 182: Bardales Ordoñez 2015, 190: MUSM 32097, 191: MUSM 28598, 195: MUSM 28600, MUSM 28601, 199: Díaz \& Linares García 2012, 200: MUSM 32095, MUSM 32096, 202: MUSM 28607-MUSM 28609, 204: MUSM 45634, 210: MUSM 28610-28616, 213: MUSM 28620, 220: MUSM 28621, 226: MUSM 32090, MUSM 32091, 227: MUSM 32092-32094, 232: MUSM 32068, MUSM 32069, 233: MUSM 28595-28597, 236: MUSM 32072, MUSM 32073, 252: Hice et al. 2004, 253: MUSM 28594, 259: MUSM 32067, 260: MUSM 28622, 264: MUSM 32103, 265: MUSM 28592, 266: MUSM 28591, 270: MUSM 28603-28606, 
276: MUSM 28617, MUSM 28618, 281: MUSM 32070, 285: MUSM 32071, 287: MUSM 32086, 290: MUSM 32085, 291: MUSM 32084, 292: MUSM 32089, 293: MUSM 32087, MUSM 32088, 294: MUSM 32083, 295: MUSM 32074-32079, 305: MUSM 32061-32065, 308: MUSM 32066, 309: MUSM 32056, MUSM 32057, 312: MUSM 32058, 314: MUSM 32059, 315: MUSM 32060, 316: Vásquez Újina 2019, 320: Ramos et al. 2017, 329: Wilson, Ascorra \& Solari 1996, 437: Bravo \& Ríos 2007. Requena.- 355: Ascorra et al. 1993. 357: Ascorra et al. 1993, 374: Voss et al. 2016, 445: Escobedo 2015. Ucayali.- 405: MUSM 17959.

\section{Glossophaga bakeri Webster y Jones, 1987}

Loreto.-35: MUSM 26935, 68: MUSM 48250, 361: MUSM 1218. Maynas.- 50 Wilson, Ascorra \& Solari 1996, 56: LSUMZ 28049, 114: TCWC 26852, 210: MUSM 28426, 231: Wilson, Ascorra \& Solari 1996, 320: Ramos et al. 2017.

\section{Glossophaga soricina (Pallas, 1766)}

Alto Amazonas.- 391: MUSM 48017. Datem del Marañón.- 17: MUSM 41727. Loreto.- 10: MUSM 41729, 14: KU 158053-158055, 22: MUSM 41728, 168: MUSM 41131, 170: MUSM 41130, 348: MUSM 47632, 350: Toffoli et al. 2018, 366: FMNH 87069, 382: MUSM 47631. Mariscal Ramón Castilla.- 84: FMNH 89112, 327: FMNH 89110, FMNH 89111. Maynas.- 3: MUSM 21162-21164, 5: MUSM 21161, 8: AMNH 73473-73475, 28: MUSM 24420, 50: Wilson, Ascorra \& Solari 1996, 54: MUSM 43005, 55: LSUMZ 28107, LSUMZ 28110, LSUMZ 28143, 62: MUSM 31869, 70: AMNH 74012, AMNH 74014, AMNH 74015, AMNH 74111, AMNH 74112, 76: MUSM 3187831880, 81: MUSM 28436, MUSM 28491, 83: MUSM 28492, MUSM 28493, 87: TCWC 27468-27470, 89: MUSM 31837-31839, 90: MUSM 28448, 94: MUSM 28511, 98: MUSM 31870-31872, 107: MUSM 28462, 109: Díaz \& Linares García 2012, 114: AMNH 98763, AMNH 98764, USNM 256819, USNM 256820, USNM 256821, TCWC 26851, 122: MUSM 31876, MUSM 31877, 123: MUSM 28510, 126: MUSM 28438, MUSM 28439, 127: Angulo \& Díaz 2004, 129: MUSM 31882-31887, 136: MUSM 28463-28466, 142: MUSM 28443, 143: MUSM 28508, MUSM 28509, 150: MUSM 31910, MUSM 31911, 151: MUSM 28470, MUSM 28471, 153: MUSM 28427, MUSM 28428, 157: MUSM 31881, 158: MUSM 31841, MUSM 31842, 159: MUSM 28534, 160: MUSM 28457, 162: MUSM 28437, 163: MUSM 28529, MUSM 28530, 164: MUSM 28531-28533, 169: MUSM 28514-28516, 173: MUSM 31909, 174: MUSM 31873-31875, 178: MUSM 28467-28469, 179: MUSM 31919-31925, 180: MUSM 28525, 181: MUSM 31916-31918, 182: Bardales Ordoñez 2015, 183: MUSM 31888, 184: MUSM 28449, MUSM 28450, 187: MUSM 28526, 188: MUSM 28524, 189: MUSM 28444-28446, 191: MUSM 28482, 192, 195: Díaz \& Linares García 2012, 197: MUSM 31915, 199: MUSM 28483-28485, 202: MUSM 28502, MUSM 28503, 205: MUSM 31843, 209: MUSM 28500, MUSM 28501, 210: MUSM 28504-28507, 211: MUSM 28517, 212: MUSM 44900, MUSM 45635, 213: MUSM 28518-28520, 220: MUSM 28521-28523, 223: MUSM 31913, MUSM 31914, 224: MUSM 31906, 227: MUSM 31907, 231: Wilson, Ascorra \& Solari 1996, 234: MUSM 28472-MUSM 28474, 235 MUSM 28475-28481, 236: MUSM 31861-31863, 237: MUSM 31864-31867, 238: MUSM 28535, 241: MUSM 28536-28540, 247: MUSM 31905, 249: MUSM 31847-31849, 250: MUSM 28452-28456, 252: Hice et al. 2004, 253: MUSM 28451, 255: MUSM 16483, 260: MUSM 28527, MUSM 28528, 265: MUSM 28440-28442, 266: MUSM 28429-28435, 268: MUSM 31912, 270: Díaz \& Linares García 2012, 271: MUSM 28447, 273: MUSM 28458-MUSM 28460, 275: MUSM 31908, 276: MUSM 28512, MUSM 28513, 278: MUSM 31850, 285: MUSM 31851-31860, 288: MUSM 31890, MUSM 31891, 292: MUSM 31898-31900, MUSM 31902-31904, 293: MUSM 31892-31895, 294: MUSM 31889, 295: MUSM 31868, 296: MUSM 31896, MUSM 31897, MUSM 31901, 305: MUSM 31844-31846, 309: MUSM 31816-31818, 310: MUSM 31819-31823, 312: MUSM 31824, MUSM 31825, 313: MUSM 31814, MUSM 31815, 314: MUSM 31826-31835, 315: MUSM 31840, 316: Vásquez Újina 2019, 318: MUSM 31836, 320: Ramos et al. 2017, 324: MUSM 21159, 329: Wilson, Ascorra \& Solari 1996, 335: MUSM 9415, MUSM 9416, 435: Bravo \& Ríos 2007. Putumayo.- 434: Bravo 2010. Requena.- 355, 357: Ascorra et al. 1993, 374: Voss et al. 2016, 399: Medina et al. 2015, 443: Escobedo 2015, 408: MUSM 20427, 411: MUSM 20426.

Glyphonycteris daviesi (Hill, 1964)

Maynas.- 65: MUSM 37654, 71: MUSM 27074, 186: MUSM 31984, 277: MUSM 31987, MUSM 31988, 282: MUSM 31986, 283: MUSM 31985, 300: MUSM 31989. Putumayo.- 434: Bravo 2010. Requena.-374: Voss et al. 2016.

\section{Glyphonycteris sy/vestris Thomas, 1896}

Maynas.- 244: MUSM 31995, 259: MUSM 31992, 268: MUSM 32001, 272: MUSM 32000, 286: MUSM 31993, MUSM 31994, 288: MUSM 31997, 293: MUSM 31999, 309: MUSM 31990, 311: MUSM 31991. Requena.- 374: MUSM 15202
Hsunycteris dashe Velazco, Soto-Centeno, Fleck, Voss and Simmons, 2017 Requena.- 374: Velazco et al 2017

Hsunycteris pattoni (Woodman y Timm, 2006)

Maynas.- 77: MUSM 31927-31930, 324: MUSM 21173. Requena.- 355: Ascorra et al. 1993, 357: MUSM 5541, MUSM 5542, MUSM 5589, 374: MUSM 15207-15210.

Hsunycteris thomasi (J. A. Allen, 1904)

Datem del Marañón.- 342: MUSM 38924, MUSM 38925, 383: Patterson \& López Wong 2014, 387: Patterson \& López Wong 2014. Loreto.- 14: Parlos et al. 2014, 35: MUSM 26936, 347: Parlos et al. 2014, 350: Toffoli et al. 2018, 361: MUSM 2991. Loreto.- 19: MUSM 41730. Maynas.- 1: MUSM 21174, 39: MUSM 27073, 49: MUSM 26572, MUSM 26573, 50: Wilson, Ascorra \& Solari 1996, 75: FMNH 87070, 81: MUSM 28558, 90: MUSM 28544-28546, 166: MUSM 31968, 173: MUSM 31967, 180: MUSM 28568, 181: MUSM 31974 182: Bardales Ordoñez 2015, 187: MUSM 28569, MUSM 28570, 190: MUSM 31966, 195: MUSM 28557, 197: MUSM 31971-31973, 202: MUSM 28561, 204: MUSM 44901, 210: MUSM 28562, MUSM 28563, 213: MUSM 28566, 217: MUSM 28567, 233: MUSM 28554-28556, 234: MUSM 28550-28553, 236: MUSM 31937-31943, 242: MUSM 31960-31965, 244: MUSM 31934 247: MUSM 31955, MUSM 31956, 248: MUSM 31957, MUSM 31958, 251: MUSM 28541, 252: Hice et al. 2004, 253: MUSM 28547, MUSM 28548, 261: MUSM 31969, MUSM 31970, 265: Díaz \& Linares García 2012, 266: MUSM 28542, 270: MUSM 28559, MUSM 28560, 273: MUSM 28549, 275: MUSM 31959, 276: MUSM 28564, MUSM 28565, 278: MUSM 31935, 279: MUSM 31947, 281: MUSM 31936, 292: MUSM 31951-31954, 294: MUSM 31948 31950, 295: MUSM 31944-31946, 315: MUSM 31931, MUSM 31932, 316: Vásquez Újina 2019, 317: MUSM 26574, 320: Ramos et al. 2017, 324: MUSM 21170-21172, 328: MUSM 31933, 329: Wilson, Ascorra \& Solari 1996, 436: Bravo \& Ríos 2007. Requena.- 355, 357: Ascorra et al. 1993, 374: Voss et al. 2016, 394, 397: Medina et al. 2015. Ucayali.- 420: MUSM 38285.

Lampronycteris brachyotis (Dobson, 1879)

Maynas.- 1: MUSM 21165 (Pacheco et al. 2009), 50: Wilson, Ascorra \& Solari 1996. Requena.- 357: Solari, Pacheco \& Vivar 1999, Gardner 2008, 374: Voss et al. 2016.

Lasiurus blossevillii (Lesson y Garnot, 1826)

Alto Amazonas.- 391: MUSM 48165. Maynas.- 159: MUSM 30027.

Lasiurus ega (Gervais, 1856)

Maynas.- 66: Pirlot 1968, 155: MUSM 33408, 159: MUSM 30028, 252: Hice et al. 2004. Ucayali.- 401: Gardner 2008.

Lionycteris spurrelli Thomas, 1913

Datem del Marañón.- 341: MUSM 16377, 343: MUSM 16378, MUSM 16379.

Loreto.- 338: MUSM 16380. Maynas.- 4: MUSM 21166-21169, 59: MUSM 26533, MUSM 26534, 260: Díaz 2011, Requena.- 443: Escobedo 2015.

Lonchorhina aurita Tomes, 1863

Ucayali.- 405: MUSM 17981.

Lophostoma brasiliense Peters, 1866

Maynas.- 1: MUSM 21195, 8: AMNH 71619, AMNH 71620, AMNH 71623 AMNH 71625-71627, 232: MUSM 32002-32004, 247: MUSM 32005, MUSM 32006, 252: Hice et al. 2004, 320: Ramos et al. 2017. Requena.- 355: Ascorra et al. 1993, 357: Wilson, Ascorra \& Solari 1996, 374: Voss et al. 2016, 399: Medina et al. 2015

Lophostoma carrikeri (J. A. Allen, 1910)

Mariscal Ramón Castilla.- 427: Escobedo 2003. Maynas.- 186: MUSM 32007 231: Wilson, Ascorra \& Solari 1996, 287: MUSM 32008, 320: Ramos et al. 2017. Putumayo: 36: MUSM 23168. Requena.- 357: Wilson, Ascorra \& Solari 1996, Fonseca \& Pinto 2004

Lophostoma silvicolum d'Orbigny, 1836

Datem del Marañón.- 67: MUSM 25862, 72: MUSM 25863, MUSM 25864 298: MUSM 43158, 304: MUSM 16396, MUSM 16397, 333: AMNH 98732, AMNH 98733, 342: MUSM 38926, 343: MUSM 16398, MUSM 16399. Loreto.- 14: KU 158142-158145, 347: KU 158138-158141, 366: FMNH 87270 FMNH 87271, FMNH 89149. Mariscal Ramón Castilla.- 84: FMNH 89036, FMNH 89037, 426, 428: Escobedo 2003. Maynas.- 1: MUSM 21183, MUSM 21184, 3: MUSM 21182, 4: MUSM 21180, MUSM 21181, 8: AMNH 7145471471, 31: MUSM 24421, 34: LSUMZ 28406, 39: MUSM 27075, 41, 50: 
Wilson, Ascorra \& Solari 1996, 66: AMNH 73524-73527, 70: AMNH 73721, AMNH 74101, AMNH 74102, 71: MUSM 27076, 77: MUSM 32009, 82: Díaz \& Linares García 2012, 90: MUSM 28575, 106: FMNH 87112, FMNH 8726587269, 180: Díaz 2011, 187: MUSM 28585, 190: MUSM 32030, 199: MUSM 28578, 202: MUSM 28581, 204: MUSM 44902, MUSM 45636, 209: MUSM 28580, 210: MUSM 28582, MUSM 28583, 233: MUSM 28577, 236: MUSM 32025, 249: MUSM 32018, 250: MUSM 28576, 251: MUSM 28572, MUSM 28573, 252: Hice et al. 2004, 255: MUSM 16498, 261: MUSM 32031, 265: MUSM 28574, 268: MUSM 32032, 274: MUSM 26535, 278: MUSM 32019, 281: MUSM 32020, 283: MUSM 32021-32024, 291: MUSM 32027, 293 MUSM 32028, MUSM 32029, 295: MUSM 32026, 305: MUSM 32014-32016, 306: MUSM 32017, 312: MUSM 32010, MUSM 32011, 315: MUSM 32013 316: Vásquez Újina 2019, 318: MUSM 32012, 320: Ramos et al. 2017, 329: Wilson, Ascorra \& Solari 1996, 330: MUSM 21176, 335: MUSM 9414, 429: Montenegro \& Escobedo 2004, 432: Bravo \& Borman 2008, 435-437: Bravo \& Ríos 2007. Putumayo.- 38: Montenegro \& Moya 2011. Requena.- 355, 357: Ascorra et al. 1993, 374: Voss et al. 2016, 379: MUSM 47657, 380: ROM 77765, 385: MUSM 47658, 411: MUSM 20428, 442: Jorge \& Velazco 2006. Ucayali.- 401: AMNH 75318, AMNH 75319, 402: MUSM 703, 412: MUSM 20429-20431, 415: Pacheco \& Arias 2001

Macrophyllum macrophyllum (Schinz, 1821)

Requena.- 374: Voss et al. 2016. Ucayali.- 405: MUSM 17978.

\section{Mesophylla macconnelli Thomas, 1901}

Alto Amazonas.- 388: Patterson \& López Wong 2014. Datem del Marañón.- 16: MUSM 41665, 45: MUSM 25865. Loreto.- 13: MUSM 41743, 14: KU 158068, KU 158069, 22: MUSM 41759, 92: MUSM 39375, 208: MUSM 48251, 347: KU 158064-158067, 350: Toffoli et al. 2018, 368: MUSM 47636. Mariscal Ramón Castilla.- 430: Montenegro \& Escobedo 2004. Maynas.- 1: MUSM 21189-21191, 2: AMNH 71495, 4: MUSM 21187, 5: MUSM 21186, 8: AMNH 71665, AMNH 71668, AMNH 71674, AMNH 71679, AMNH 71686, 28: MUSM 24422, 32: LSUMZ 28440, 49: MUSM 26546, MUSM 26582, 55: LSUMZ 28030-28044, LSUMZ 28270, LSUMZ 28271, 57: MUSM 43006, 59: MUSM 26581, 63: LSUMZ 28441-28444, 70: AMNH 74100, 75: FMNH 8708587096, 173: MUSM 32866, 176: USNM 337942-337945, 179: MUSM 32872 181: MUSM 32869-32871, 182: Bardales Ordoñez 2015, 188: MUSM 29575 190: MUSM 32865, 202: MUSM 29570-29572, 210: MUSM 29573, 218: MUSM 32862-32864, 220: MUSM 29574, 223: MUSM 32868, 226: MUSM 32861, 247: MUSM 32859, MUSM 32860, 250: MUSM 29569, 251: MUSM 29564-29566, 252: Hice et al. 2004, 253: MUSM 29567, MUSM 29568, 259. MUSM 32841, 260: Díaz 2011, 263: MUSM 32867, 279: MUSM 32849, 280: MUSM 32846, MUSM 32847, MUSM 33251, 281: MUSM 32842-32844, 282: MUSM 32845, 287: MUSM 32853, MUSM 32854, 288: MUSM 32852, 292: MUSM 32855, MUSM 32858, 294: MUSM 32851, 295: MUSM 32848, 296: MUSM 32856, MUSM 32857, 306: MUSM 32839, MUSM 32840, 311: MUSM 32837, 312: MUSM 32833, 314: MUSM 32834, 315: MUSM 32838, 316: Vásquez Újina 2019, 317: MUSM 26547, 318: MUSM 32835, MUSM 32836, 320: Ramos et al. 2017, 323: MUSM 32873, 329: Wilson, Ascorra \& Solar 1996, 332: MUSM 32850, 433: Bravo 2010. Putumayo.- 434: Bravo 2010. Requena.- 355: MUSM 5935, MUSM 5936, MUSM 5941, 357: Ascorra et al. 1993, 367: FMNH 89052, 374: Voss et al. 2016, 394, 395, 398, 399: Medina et al. 2015, 408: MUSM 20434-20436, 411: MUSM 20432, MUSM 20433, 440: Jorge \& Velazco 2006. Ucayali.- 401: AMNH 76254, AMNH 76255, 405: MUSM 17906, MUSM 17942, MUSM 17954, MUSM 17999, MUSM 18004, 412: MUSM 20437, MUSM 20438, 420: MUSM 38286.

Micronycteris (Leuconycteris) brosseti Simmons y Voss, 1998

Loreto.- 69: MUSM 48252. Mariscal Ramón Castilla.- 327: Simmons et al. 2002. Maynas.- 66: Simmons et al. 2002. Requena.- 357: Simmons et al. 2002.

Micronycteris (Xenoctenes) hirsuta (Peters, 1869)

Datem del Marañón.- 304: MUSM 16381, 336: MUSM 38927. Requena.374: Voss et al. 2016

Micronycteris (Micronycteris) matses Simmons, Voss, y Fleck, 2002 Requena.- 374: Simmons et al. 2002, 397: Medina et al. 2015.

Micronycteris (Micronycteris) megalotis (Gray, 1842)

Datem del Marañón.- 67: MUSM 25866, 383: Patterson \& López Wong 2014, 387: Patterson \& López Wong 2014. Loreto.- 19: MUSM 41731, 96 MUSM 26943. Mariscal Ramón Castilla.- 84: FMNH 89105, FMNH 89106, FMNH 89171, 327: Simmons et al. 2002. Maynas.- 40: MUSM 21193, MUSM 21194, 41: Wilson, Ascorra \& Solari 1996, 59: MUSM 26536, 66: Simmons et al. 2002, 182: Bardales Ordoñez 2015, 195: Díaz \& Linares García 2012
199: MUSM 28587, 252: Hice et al. 2004, 253: MUSM 28586, 260: Díaz \& Linares García 2012, 264: MUSM 32040-32042, 295: MUSM 32038, 300: MUSM 32039, 305: MUSM 32033-32035, 308: MUSM 32036, 317: MUSM 26575, 320: Ramos et al. 2017, 323: MUSM 32043, 328: MUSM 32037, 329: Wilson, Ascorra \& Solari 1996. Putumayo.- 15: Montenegro \& Moya 2011. Requena.- 355: MUSM 5913, 357: Wilson, Ascorra \& Solari 1996, 374: Moras, Gomes \& Tavares 2014. Ucayali.- 402: MUSM 701, 405: MUSM 17992.

Micronycteris (Micronycteris) microtis Miller, 1898

Loreto.- 29: MUSM 41617. Requena.- 374: Moras, Gomes \& Tavares 2014, Voss et al. 2016.

Micronycteris (Schizonycteris) minuta (Gervais, 1856)

Datem del Marañón.- 33: MUSM 37786. Maynas.- 4: MUSM 21197, 8: Simmons et al. 2002, 139: MUSM 28590, 186: MUSM 32044, 223: MUSM 32051, 231: Wilson, Ascorra \& Solari 1996, 248: MUSM 32049, 252: Hice et al. 2004, 258: MUSM 32046-32048, 268: MUSM 32050, 311: MUSM 32045, 320: Ramos et al. 2017. Requena.- 357: Ascorra et al. 1993, 374: Voss et al. 2016.

Molossops neglectus Williams y Genoways, 1980

Maynas.- 172: MUSM 30920-30923, 212: MUSM 44837, 303: MUSM 30919. Requena.- 357: Ascorra et al. 1993.

Molossops temminckii (Burmeister, 1854)

Loreto.- 361: MUSM 799. Maynas.- 8: Gardner 2008, 204: MUSM 44838 , 252: Hice et al. 2004

Molossus alvarezi González-Ruiz, Ramírez-Pulido \& Arroyo-Cabrales, 2011 Maynas.- 42: MUSM 20986, 50: Wilson, Ascorra \& Solari 1996, 114: MUSM 16302.

Molossus coibensis J. A. Allen, 1904

Maynas.- 97: Díaz \& Linares García 2012, 116, 119: Díaz 2011, 120: Díaz \& Linares García 2012, 151: Díaz 2011.

Molossus molossus (Pallas, 1766)

Alto Amazonas.- 390: FMNH 19953. Datem del Marañón.- 256: FMNH 89023-89025, 298: MUSM 43042, 371: MUSM 43043. Loreto.- 14: KU 157989-157992, 24: MUSM 41604, 349: KU 157984-157988. Mariscal Ramón Castilla.- 44: FMNH 95909-95912. Maynas.- 40: MUSM 20985, 43: FMNH 34240-34242, 54: MUSM 42968, 66: AMNH 73505-73522, 83: MUSM 27297-27302, 97, 99: Díaz \& Linares García 2012, 100: MUSM 2733027334, 101, 103: Díaz \& Linares García 2012, 105: MUSM 30928-30931, 108: MUSM 27274-27280, 110: MUSM 27264, MUSM 27265, 113: MUSM 30924, 114: AMNH 98722, AMNH 98762, 116: MUSM 27271, MUSM 27272, 117: MUSM 20984, 118: MUSM 27273, 120: MUSM 27252-27260, 124: Díaz \& Linares García 2012, 127: Angulo \& Díaz 2004, 128: Díaz \& Linares García 2012, 132: MUSM 27303-27305, 133: FMNH 87008, 134: MUSM 27284, 136: MUSM 27281-27283, 144: MUSM 27240-27242, 148: Díaz \& Linares García 2012, 151: MUSM 27286-27289, 153: MUSM 27318-27322, 154: MUSM 27191-27193, 160: Díaz \& Linares García 2012, 162: MUSM 27187-27190, 165: Díaz 2011, 166: MUSM 30947-30951, 171: MUSM 30946, 172: MUSM 30939-30945, 183: MUSM 30932, MUSM 30933, 185: FMNH 122944-122951, 193: Díaz \& Linares García 2012, 196: MUSM 27285, 198: TCWC 27557, 204: MUSM 44844-44854, 205: MUSM 30925-30927, 207: MUSM 44839-44842, 212: MUSM 44843, 219: MUSM 30934-30938, 235: MUSM 27290, 252: Hice et al. 2004, 264: MUSM 30952-30954, 273: MUSM 27243, MUSM 27244, 324: MUSM 20983, 329: Wilson, Ascorra \& Solari 1996. Requena.- 355: MUSM 6663-6666, 357: Wilson, Ascorra \& Solari 1996, Tello et al. 2013, 374: Voss et al. 2016, 379: MUSM 47604, 394, 395: Medina et al. 2015, 411: MUSM 20394-20397. Ucayali.- 401: AMNH 76205-76218, AMNH 76250, 403: MUSM 4821.

Molossus rufus E. Geoffroy, 1805

Maynas.- 8: AMNH 71396, 66: AMNH 73976-73983, 104: MUSM 2734027346, 105: MUSM 30962-30965, 112: MUSM 27348, 113: MUSM 3095530961, 115: MUSM 27339, 121: MUSM 27347, 136: Díaz \& Linares García 2012, 138: MUSM 42392, 154: MUSM 27335-27338, 172: MUSM 30966, 212: MUSM 44855, 252: Hice et al. 2004. Requena.- 357: Wilson, Ascorra \& Solari 1996, 374: Voss et al. 2016, 394, 398: Medina et al. 2015. Ucayali.401: AMNH 75301- 75317.

\section{Myotis albescens (E. Geoffroy, 1806)}

Alto Amazonas.- 389: MUSM 48024, MUSM 48025, 391: MUSM 48166 48168, Loreto.- 347: KU 158160, 368: MUSM 38425, MUSM 47665. Maynas.- 
1: MUSM 21339, MUSM 21340, 3: MUSM 21338, 4: MUSM 21333-21337, 8: AMNH 71643, AMNH 71644, 50: Wilson, Ascorra \& Solari 1996, 66: AMNH 73233-73242, 70: AMNH 74017-74021, 110: MUSM 29963, 111: MUSM 29979, 116: MUSM 29968-29978, 125: MUSM 29964-29967, 139: MUSM 29982, 166: MUSM 33354, MUSM 33355, 181: MUSM 33356, MUSM 33357, 183: MUSM 33352, MUSM 33353, 184: Díaz \& Linares García 2012, 191: MUSM 29980, MUSM 29981, 252: Hice et al. 2004, 273: MUSM 29962, 335: MUSM 9435. Requena.- 362: MUSM 5508, 374: MUSM 15240. Ucayali.- 393: FMNH 55418, 401: AMNH 76238

Myotis keaysi J. A. Allen, 1914

Datem del Marañón.- 341: MUSM 16383, 383: Patterson \& López Wong 2014. Maynas.- 255: MUSM 16499.

Myotis nigricans (Schinz, 1821)

Alto Amazonas.- 390: FMNH 19957, 391: MUSM 48169. Datem del Marañón.- 25: MUSM 39751, 256: FMNH 89125, 373: AMNH 98790. Loreto.- 14: KU 158161. Mariscal Ramón Castilla.- 44: FMNH 95908. Maynas.- 41, 50: Wilson, Ascorra \& Solari 1996, 66: AMNH 73232, 76: MUSM 33375, 80: MUSM 4356, 105: MUSM 33371, 106: FMNH 87006, FMNH 87007, 114: FMNH 89126, 122: MUSM 33368-33370, 125: MUSM 29983, 129: MUSM 33376-33379, 137: MUSM 33372-33374, 149: MUSM 33380, 150: MUSM 33386, 151: MUSM 29984, 166: MUSM 33387, 172: MUSM 33383-33385, 179: MUSM 33392, 197: MUSM 33391, 223: MUSM 33389, MUSM 33390, 225: MUSM 43015-43017, 237: MUSM 33365, 243: MUSM 33360, 249: MUSM 33361, 252: Hice et al. 2004, 255: MUSM 16500, 261: MUSM 33388, 281: MUSM 33362-33364, 287: MUSM 33382, 290: MUSM 33381, 295: MUSM 33366, MUSM 33367, 315: MUSM 33358, MUSM 33359, 320: Ramos et al. 2017, 323: MUSM 33393, 329: Wilson, Ascorra \& Solari 1996, 435: Bravo \& Ríos 2007. Putumayo.- 23: Montenegro \& Moya 2011. Requena.- 355: MUSM 5512, 357: Ascorra et al. 1993, 374: Voss et al. 2016. Ucayali.- 401: AMNH 76239, 405: MUSM 17983, 422: MCZ 23931-23933.

\section{Myotis riparius Handley, 1960}

Loreto.- 12: MUSM 41629, 347: KU 158162. Maynas.- 1: MUSM 21344 8: AMNH 71639-71642, AMNH 71645, AMNH 71646, USNM 254648, 50: Wilson, Ascorra \& Solari 1996, 76: MUSM 33395, 83: MUSM 30007, MUSM 30008, 88: Díaz \& Linares García 2012, 93: MUSM 30012, 94: Díaz \& Linares García 2012, 115: MUSM 29989, MUSM 29990, 126: MUSM 29991, MUSM 29992, 127: Angulo \& Díaz 2004, 152: MUSM 29994-29996, 171: MUSM 33398, 174: MUSM 33394, 178: MUSM 30000, 190: MUSM 33397, 191: MUSM 30004, MUSM 30005, 194: MUSM 33396, 195: MUSM 30006, 196: MUSM 29998, MUSM 29999, 197: MUSM 33399, 210: MUSM 30009, 235: MUSM 30001-MUSM 30003, 238: MUSM 30018, 241: MUSM 30019, MUSM 30020, 251: MUSM 29985-29987, 252: Hice et al. 2004, 253: MUSM 29997 260: Díaz 2011, 265: MUSM 29993, 266: MUSM 29988, 276: MUSM 30016, 316: Vásquez Újina 2019, 320: Ramos et al. 2017, 329: Wilson, Ascorra \& Solari 1996, 330: MUSM 21342, MUSM 21343. Requena.- 355: MUSM 6662, 357: Wilson, Ascorra \& Solari 1996, 374: Voss et al. 2016.

\section{Myotis simus Thomas, 1901}

Maynas.- 3: MUSM 21346, 8: AMNH 71483-71494, USNM 254649, 61: AMNH 74378-74381, 70: AMNH 74105, AMNH 74109, AMNH 74110, 151: MUSM 30022, 162: MUSM 30021, 183: MUSM 33400, 252: Hice et al. 2004 329: Wilson, Ascorra \& Solari 1996, 330: MUSM 21345. Requena.- 357: Wilson, Ascorra \& Solari 1996. Ucayali.- 401: AMNH 76240-76249, AMNH 76252, AMNH 76253, 404: AMNH 98723, 418: AMNH 98730.

\section{Noctilio albiventris Desmarest, 1818}

Alto Amazonas.- 389: MUSM 48004, 390: FMNH 19859, 391: MUSM 48001 48003, MUSM 48120-48130. Loreto.- 35: MUSM 26934, 321: MUSM 1106 , 325: FMNH 122832, 337: KU 140190, 361: MUSM 1105, MUSM 3044, 363: MUSM 47606, 365: FMNH 122830, FMNH 122831. Mariscal Ramón Castilla.- 84: FMNH 89016-89018, 327: FMNH 89013, FMNH 89014, 331: FMNH 89015. Maynas.- 1: MUSM 20988-20991, 4: MUSM 20987, 8: AMNH 71397, AMNH 71398, AMNH 71475-71482, 56: LSUMZ 28045-28048, 94: MUSM 27380, 110: Díaz \& Linares García 2012, 111: MUSM 27377, MUSM 27378, 125: MUSM 27372-27376, 126: MUSM 27355- 27358, 127: Angulo \& Díaz 2004, 132: MUSM 27379, 139: MUSM 27381-27383, 166: MUSM 30974-30976, 172: MUSM 30973, 182: Bardales Ordoñez 2015, 183: MUSM 30969-30972, 252: Hice et al. 2004. Requena.- 346: USNM 241113, 355: Ascorra et al. 1993, 357: Wilson, Ascorra \& Solari 1996, 374: Voss et al. 2016. Ucayali.- 421: AMNH 169947

Noctilio leporinus (Linnaeus, 1758)

Loreto.- 14: KU 157994. Mariscal Ramón Castilla.- 327: FMNH 89002-89012,
FMNH 89161-89164. Maynas.- 66: AMNH 73523, AMNH 73925-73975, 114: AMNH 99311, 191: MUSM 27384, 432: Bravo \& Borman 2008.

Peropteryx kappleri Peters, 1867

Maynas.- 49: MUSM 26566. Requena.- 357: MUSM 6976, 374: Lim et al. 2010.

Peropteryx leucoptera Peters, 1867

Mariscal Ramón Castilla.- 327: FMNH 89103, FMNH 89104. Maynas.- 28: MUSM 24412, 40: MUSM 20973, MUSM 6744, 41: Wilson, Ascorra \& Solari 1996, 186: MUSM 30852, MUSM 30853, 226: MUSM 30862, 252: Hice et al. 2004, 265: MUSM 27144-27147, 276: Díaz \& Linares García 2012, 281: MUSM 30855-30858, 294. MUSM 30859-30861, 311: MUSM 30854. Requena.- 374: Lim et al. 2010

Peropteryx macrotis (Wagner, 1843)

Loreto.- 27: MUSM 41676. Maynas.- 4: MUSM 20974, 66: Lim et al. 2010, 116: Díaz 2011, 277: MUSM 30866, 284: MUSM 30865, 286: MUSM 30864, 300: MUSM 30867, 311: MUSM 30863, 320: Ramos et al. 2017, 335: MUSM 9410-MUSM 9412. Requena.- 374: Voss et al. 2016.

Peropteryx pallidoptera Lim, Engstrom, Reid, Simmons, Voss \& Fleck, 2010 Maynas.- 70: Lim et al. 2010, 265: Díaz 2011. Requena.- 372, 374: Lim et al. 2010, 399: Medina et al. 2015.

Phylloderma stenops Peters, 1865

Loreto.- 14: KU 158072, KU 158073, 359: MUSM 47637. Maynas.- 4: MUSM 21201, 55: LSUMZ 28279, 59: MUSM 26576, 186: MUSM 32104, 222: MUSM 32109, 232: MUSM 32107, 252: Hice et al. 2004, 269: MUSM 32108, 313: MUSM 32105, 318: MUSM 32106, 322: MUSM 32110, 332: MUSM 32111. Requena.- 354: MUSM 5495, 355: MUSM 5916, 357: Wilson, Ascorra \& Solari 1996

\section{Phyllostomus discolor Wagner, 1843}

Alto Amazonas.- 391: MUSM 48018, MUSM 48019, MUSM 48159. Loreto.368: MUSM 47638. Mariscal Ramón Castilla.- 427, 428: Escobedo 2003. Maynas.- 54: MUSM 43009, 57: MUSM 43007, MUSM 43008, 62: MUSM 32116, 81: MUSM 28623, 87: TCWC 27471, TCWC 27472, 98: MUSM $32117-$ 32121, 100: MUSM 28648, 105: MUSM 32124, 122: MUSM 32123, 126 MUSM 28625, MUSM 28626, 127: Angulo \& Díaz 2004, 129: MUSM $32127-$ 32130, 135: MUSM 28624, 149: MUSM 32131, MUSM 32132, 150: MUSM 32142, 151: MUSM 28630-28638, 152: MUSM 28627, MUSM 28628, 153: MUSM 28643-28647, 155: MUSM 32125, MUSM 32126, 158: MUSM 32114 MUSM 32115, 174: MUSM 32122, 179: MUSM 32145, MUSM 32146, 183: MUSM 32133-32135, 196: MUSM 28629, 197: MUSM 32144, 210: MUSM 28641, MUSM 28642, 225: MUSM 43010, MUSM 43011, 231: Wilson, Ascorra \& Solari 1996, 235: MUSM 28639, MUSM 28640, 242: MUSM 32140, MUSM 32141, 252: Hice et al. 2004, 267: MUSM 32143, 292: MUSM 32136, MUSM 32138, MUSM 32139, 296: MUSM 32137, 314: MUSM 32112, MUSM 32113, 316: Vásquez Újina 2019. Requena.- 355, 357: Ascorra et al. 1993.

Phyllostomus elongatus (E. Geoffroy, 1810)

Alto Amazonas.- 388: Patterson \& López Wong 2014. Datem del Marañón.16: MUSM 41658, 37: MUSM 25867, 438: Castro Vergara 2012. Loreto.- 11: MUSM 41659, 14: KU 158077-158084, 29: MUSM 41618, 347: KU 158074158076, 350: Toffoli et al. 2018, 368: MUSM 38404, MUSM 38405, MUSM 47641, MUSM 47642. Mariscal Ramón Castilla.- 327: FMNH 89041, FMNH 89042, FMNH 89150, 426, 427: Escobedo 2003. Maynas.- 1: MUSM 2120921212, 3: MUSM 21208, 4: MUSM 21206, MUSM 21207, 8: AMNH 7170971717, 39: MUSM 27077, 40: MUSM 6747, 41, 50: Wilson, Ascorra \& Solari 1996, 55: LSUMZ 28280, 56: LSUMZ 28278, 58: MUSM 37656, 66: AMNH 73528, 77: MUSM 32148, 87: TCWC 27473, 106: FMNH 87113- 87115, 151: MUSM 28653, 178: MUSM 28652, 182: Bardales Ordoñez 2015, 186: MUSM 32147, 190: MUSM 32160, MUSM 32161, 218: MUSM 32158, MUSM 32159, 231: Wilson, Ascorra \& Solari 1996, 248: MUSM 32157, 252: Hice et al. 2004 261: MUSM 32162, 265: MUSM 28649, MUSM 28650, 268: MUSM 32163, 269: MUSM 32150, MUSM 32151, 276: MUSM 28654, 280: MUSM 32153, 283: MUSM 32152, 291: MUSM 32155, 293: MUSM 32156, 295: MUSM 32154, 309: MUSM 32149, 317: MUSM 26537, 320: Ramos et al. 2017 329: Wilson, Ascorra \& Solari 1996, 335: MUSM 9413, 429: Montenegro \& Escobedo 2004, 432: Bravo \& Borman 2008, 433: Bravo 2010, 436, 437 Bravo \& Ríos 2007. Putumayo.- 15, 30: Montenegro \& Moya 2011, 431: Montenegro \& Escobedo 2004. Requena.- 354: MUSM 5494, 355: Ascorra et al. 1993, 357: Wilson, Ascorra \& Solari 1996, 374: Voss et al. 2016, 379: MUSM 47639, MUSM 47643, 384: Díaz \& Linares García 2012, 392: MUSM 47640, 397: Medina et al. 2015, 408: MUSM 20439-20441, 440: Jorge \& 
Velazco 2006, 443, 445: Escobedo 2015. Ucayali.- 401: AMNH 76165-76168, 405: MUSM 17912, 424: Pacheco \& Arias 2001.

Phyllostomus hastatus (Pallas, 1767)

Alto Amazonas.- 391: MUSM 48020. Datem del Marañón.- 37: MUSM 25868, 45: MUSM 25870, 67: MUSM 25869, 298: MUSM 43159, MUSM 43160. Loreto.- 14: KU 158088-158093, 347: KU 158085-158087, 361: MUSM 1170, 382: MUSM 47644. Maynas.- 8: AMNH 71399, AMNH 71400, AMNH 71472, 41, 50: Wilson, Ascorra \& Solari 1996, 58: MUSM 37655, 59: MUSM 26577, 62: MUSM 32207, 66: AMNH 73529-73534, AMNH 73986-73988, 70: AMNH 74098, AMNH 74099, 76: MUSM 32208, 81: MUSM 28690, MUSM 28691, 90: MUSM 28664, 104: MUSM 28675, MUSM 28676, 106 FMNH 87063-87067, 139: MUSM 28727, MUSM 28728, 148: Díaz \& Linares García 2012, 151: MUSM 28677, 158: MUSM 32178, 160: MUSM 28668, 169: MUSM 28720, 172: MUSM 32256-32258, 177: MUSM 21213, 180: MUSM 28729, MUSM 28730, 182: Bardales Ordoñez 2015, 195: MUSM 28688, MUSM 28689, 197: MUSM 32259, 199: MUSM 28685-28687, 202: MUSM 28704, MUSM 28705, 205: MUSM 32179-32184, 209: MUSM 28699-28703, 210: MUSM 28706, 214: MUSM 32254, 218: MUSM 32251, 220: Díaz \& Linares García 2012, 226: MUSM 32250, 229: MUSM 32253, 230: MUSM 32252, 231: Wilson, Ascorra \& Solari 1996, 233: MUSM 28678, 235: MUSM 28679-28684, 236: MUSM 32197, MUSM 32198, 237: MUSM 32199-32205, 242: MUSM 32255, 247: MUSM 32249, 249: MUSM 32185, MUSM 32186, 250: MUSM 28665-28667, 251: MUSM 28655-28657, 252 Hice et al. 2004, 255: MUSM 16484-16487, 257: MUSM 32164, 258: MUSM 32209, 262: Díaz \& Linares García 2012, 265: MUSM 28660-28662, 270: MUSM 28692-28698, 271: MUSM 28663, 273: MUSM 28669-28674, 276: MUSM 28719, 281: MUSM 32187, 282: MUSM 32188, MUSM 32189, 285 MUSM 32190-32196, 287: MUSM 32219-32225, 288: MUSM 32214-32218, 291: MUSM 32211-32213, 292: MUSM 32240-32243, MUSM 32246-32248, 293: MUSM 32226-32234, 295: MUSM 32206, 296: MUSM 32235-32239, MUSM 32244, MUSM 32245, 309: MUSM 32165-32168, 312: MUSM 32169, MUSM 32170, 314: MUSM 32171-32173, 316: Vásquez Újina 2019, 318: MUSM 32174-32177, 320: Ramos et al. 2017, 322: MUSM 32210, 329: Wilson, Ascorra \& Solari 1996, 429: Montenegro \& Escobedo 2004, 433 : Bravo 2010. Putumayo.- 38: Montenegro \& Moya 2011. Requena.- 355: Ascorra et al. 1993, 356: KU 143593, 357: Wilson, Ascorra \& Solari 1996 , 374: Voss et al. 2016, 394, 395, 398, 399: Medina et al. 2015, 440: Jorge \& Velazco 2006, 444: Escobedo 2015. Ucayali.- 401: AMNH 75296-75300, 406: AMNH 98715, AMNH 98716.

Phyllostomus latifolius (Thomas, 1901)

Maynas.- 4: Pacheco et al. 2009.

\section{Platyrrhinus brachycephalus (Rouk y Carter, 1972)}

Alto Amazonas.- 390: Velazco 2005, 391: MUSM 48021-48023, MUSM 48160-48162. Datem del Marañón.- 26: MUSM 41666, 256: Velazco 2005, 298: MUSM 43161, MUSM 43162, 341: MUSM 16384, 343: MUSM 16402. Loreto.- 10: MUSM 41744, MUSM 41745, 11: MUSM 41667, MUSM 41668, 12: MUSM 41621, MUSM 41622, 14: KU 158094, KU 158095, 325: Velazco 2005, 337: KU 140195, KU 140196, 344: Toffoli et al. 2018, 345: MUSM 38410, 350: Toffoli et al. 2018, 361: MUSM 3169, 365: Velazco 2005. Mariscal Ramón Castilla.- 84: Velazco 2005, 327: Velazco 2005. Maynas.- 4: MUSM 21219, MUSM 21231, 40, 46: Velazco 2005, 53: MUSM 43012, 57: MUSM 43013, 62: MUSM 32885-32887, 76: MUSM 32889, 77: MUSM 32874, MUSM 32875, 90: MUSM 29590-29593, 123: MUSM 29609, 129: MUSM 32891, 130 MUSM 32890, 135: MUSM 29578, MUSM 29579, 139: MUSM 29613, MUSM 29614, 146: MUSM 29583-29589, 149: MUSM 32892, MUSM 32893, 150 MUSM 32901, 156: MUSM 29580-29582, 158: MUSM 32878, 159: MUSM 29616, 160: MUSM 29594, MUSM 29595, 166: MUSM 32902, 169: MUSM 29611, MUSM 29612, 174: MUSM 32888, 178: MUSM 29599- 29601, 180: MUSM 29615, 181: MUSM 32903, 183: MUSM 32895, 196: MUSM 2959629598, 202: MUSM 29608, 206: MUSM 32900, 209: MUSM 29606, MUSM 29607, 221: TCWC 29566-29571, 222: MUSM 32894, 231: Wilson, Ascorra \& Solari 1996, 234: MUSM 29602, 235: MUSM 29603-29605, 240: ROM 77317, 241: MUSM 29617, MUSM 29618, 252: Hice et al. 2004, 255: MUSM 16488-16493, 258: MUSM 32896, 276: MUSM 29610, 281: MUSM 32879, 282: MUSM 32880, 285: MUSM 32881, MUSM 32882, 292: MUSM 32899, 295: MUSM 32883, MUSM 32884, 296: MUSM 32897, MUSM 32898, 310 MUSM 32876, 312: MUSM 32877, 316: Vásquez Újina 2019, 329: Wilson, Ascorra \& Solari 1996. Requena.- 355: Velazco 2005, 357: Wilson, Ascorra \& Solari 1996, 397: Medina et al. 2015, 398, 399: Medina et al. 2015, 441: Jorge \& Velazco 2006. Ucayali.- 420: MUSM 38287.

Platyrrhinus fusciventris Velazco, Gardner y Patterson, 2010 Requena.- 354, 355: Velazco 2005, Velazco et al. 2021.
Platyrrhinus incarum (Thomas, 1912)

Alto Amazonas.- 388: Patterson \& López Wong 2014, 390: Sanborn 1949b Datem del Marañón.- 25: MUSM 39752, MUSM 39753, 342: MUSM 38928. Loreto.- 10: MUSM 41746, 14: KU 158096, 325: FMNH 122879, 365: FMNH 122872, FMNH 122873, FMNH 122877, 382: MUSM 47645. Mariscal Ramón Castilla.- 44: FMNH 104772-104775, FMNH 104867, FMNH 104869, 327: Velazco 2005. Maynas.- 1: MUSM 21220, MUSM 21233, MUSM 21234, 4: MUSM 21226-21230, MUSM 21232, 40: MUSM 21223-21225, 41: Wilson, Ascorra \& Solari 1996, 46: Velazco 2005, 50: Wilson, Ascorra \& Solari 1996, 64: AMNH 98770, AMNH 98782, 70: AMNH 73990-73993, AMNH 74013, 83: MUSM 29644, 90: MUSM 29623-29625, 130: MUSM 32930, 164: MUSM 29664, 176: USNM 337941, 180: MUSM 29657-29659, 183: MUSM 32931, 184: MUSM 29626, 187: MUSM 29660, 188: MUSM 29656, 195: MUSM 29643, 197: MUSM 32948-32950, 199: MUSM 29641, MUSM 29642, 202: MUSM 29648-29650, 204: MUSM 44905, MUSM 45644, MUSM 45645, 207: MUSM 44903, 209: MUSM 29647, 210: MUSM 29651, MUSM 29652, 212: MUSM 44904, MUSM 45637-45643, 213: MUSM 29655, 221: TCWC 2957229578, 226: MUSM 32944, 233: MUSM 29635-29639, 234: MUSM 2962929634, 235: MUSM 29640, 237: MUSM 32926-32928, 240: ROM 77318, TCWC 27553-27556, 241: MUSM 29665-29668, 242: MUSM 32945-32947, 246: MUSM 32943, 247: MUSM 32942, 251: MUSM 29619, 252: Hice et al. 2004, 253: MUSM 29627, 260: MUSM 29661-MUSM 29663, 266: MUSM 29620-MUSM 29622, 270: MUSM 29645, MUSM 29646, 273: MUSM 29628, 276: MUSM 29653, MUSM 29654, 279: MUSM 32932, 280: MUSM 32923, 282: MUSM 32921, MUSM 32922, 285: MUSM 32924, MUSM 32925, 287: MUSM 32938, 288: MUSM 32935-32937, 291: MUSM 32933, MUSM 32934, 292: MUSM 32941, 293: MUSM 32939, MUSM 32940, 295: MUSM 32929, 307: MUSM 32920, 309: MUSM 32904, MUSM 32905, 310: MUSM 32906, MUSM 32907, 311: MUSM 32919, 312: MUSM 32908-32913, 314: MUSM 32914-32918, 316: Vásquez Újina 2019, 323: MUSM 32951, 330: MUSM 21221, 335: Velazco 2005, 437: Bravo \& Ríos 2007. Requena.- 354: Velazco 2005, 357: Wilson, Ascorra \& Solari 1996, 394, 395, 398, 399: Medina et al. 2015, 441: Jorge \& Velazco 2006.

\section{Platyrrhinus infuscus (Peters, 1880)}

Datem del Marañón.- 297: MUSM 37707, 439: Castro Vergara 2012. Loreto.- 12: MUSM 41623, 325: Velazco 2005. Maynas.- 1: MUSM 21235, 8: Velazco 2005, 76: MUSM 32953, 252: Hice et al. 2004, 310: MUSM 32952, 322: MUSM 32954. Requena.- 374: Voss et al. 2016, 411: MUSM 20442, MUSM 20443, 440: Jorge \& Velazco 2006. Ucayali.- 405: MUSM 17944, MUSM 17951, MUSM 17967, MUSM 17968, MUSM 17976, MUSM 18020.

Promops centralis Thomas, 1915

Maynas.- 108: MUSM 27353, MUSM 27354, 113: MUSM 30967, MUSM 30968, 212: MUSM 44856, 252: Hice et al. 2004. Requena.- 357: Wilson, Ascorra \& Solari 1996.

\section{Rhinophylla fischerae Carter, 1966}

Alto Amazonas.- 388: Patterson \& López Wong 2014. Datem del Marañón.256: FMNH 89051. Loreto.- 14: KU 158102-158106, 21: MUSM 41717, 347: KU 158097-158101, Loreto.- 10: MUSM 41716, 19: MUSM 41713, MUSM 41714, 22: MUSM 41715. Maynas.- 1: MUSM 21240-21242, 3: MUSM 21238, MUSM 21239, 39: MUSM 27072, 40: MUSM 21237, 41: Wilson, Ascorra \& Solari 1996, 55: LSUMZ 28054, LSUMZ 28061-28064, 58: MUSM 37657, 59: MUSM 26569, 76: MUSM 31599, MUSM 31600, 77: MUSM 31584, MUSM 31585, 199: MUSM 28246, 202: MUSM 28250, 206: MUSM 31612, 212: MUSM 45646, 218: MUSM 31610, 230: MUSM 31611, 236: MUSM 31597, MUSM 31598, 252: Hice et al. 2004, 253: MUSM 28244, 260: Díaz 2011, 261: MUSM 31613, MUSM 31614, 270: MUSM 28247-28249, 273: MUSM 28245, 274: MUSM 26525, 279: MUSM 31601, 281: MUSM 31596, 287: MUSM 31604, 288: MUSM 31603, 292: MUSM 31609, 293: MUSM 31605, MUSM 31606, 294: MUSM 31602, 296: MUSM 31607, MUSM 31608, 309: MUSM 31587, 310: MUSM 31588, 312: MUSM 31589, MUSM 31590, 313: MUSM 31586, 314: MUSM 31591, 315: MUSM 31593, 316: Vásquez Újina 2019, 317: MUSM 26526, 318: MUSM 31592, 320: Ramos et al. 2017, 323: MUSM 31615, 328: MUSM 31594, MUSM 31595, 329: Wilson, Ascorra \& Solari 1996. Requena.- 357: Wilson, Ascorra \& Solari 1996, 374: Voss et al. 2016, 394, 398, 399: Medina et al. 2015, 445: Escobedo 2015.

\section{Rhinophylla pumilio Peters, 1865}

Alto Amazonas.- 388: Patterson \& López Wong 2014. Datem del Marañón.16: MUSM 41650-41653, 17: MUSM 41718, 37: MUSM 25872, 67: MUSM 25873, 73: MUSM 25871, 304: MUSM 16386, 342: MUSM 38929. Loreto.11: MUSM 41654, 41655, 14: KU 158116-158121, 29: MUSM 41615-41616, 345: MUSM 47649, 347: KU 158107-158115, 361: MUSM 1521, 368: MUSM 
47650-47651, 13: MUSM 41722, 19: MUSM 41719, 22: MUSM 41720-41721. Mariscal Ramón Castilla.- 430: Montenegro \& Escobedo 2004. Maynas.-1: MUSM 21255-21264, 3: MUSM 21252-21254, 4: MUSM 21249-21251, 5: MUSM 21248, 8: AMNH 71657-71687, USNM 252761-252762, 28: MUSM 24425-24426, 31: MUSM 24424, 40: MUSM 21247, 6751, 41: Wilson, Ascorra \& Solari 1996, 49: MUSM 26528, 50: Wilson, Ascorra \& Solari 1996, 52: MUSM 37661-37662, 55: LSUMZ 28050-28060, 58: MUSM 37663, 59: MUSM 26527, 26570, 60: MUSM 37664, 37666, 63: LSUMZ 28434-28435, 66: AMNH 73460-73472, 73477, 73478, 73479, 73481, 74: MUSM 37658-37660, 76: MUSM 31711-31712, 77: MUSM 31616-31617, 81: MUSM 28334-28337, 83: MUSM 28338-28340, 90: MUSM 28270-28274, 169: MUSM 28385, 171: MUSM 31765, 173: MUSM 31766, 175: MUSM 28420, 177: MUSM 28380, 178: MUSM 28294-28297, 180: MUSM 28398-28411, 181: MUSM 31775, 187: MUSM 28412-28414, 188: MUSM 28391-28397, 191: MUSM 28330, 195: MUSM 28332-28333, 197: MUSM 31773-31774, 199: MUSM 28331, 202: MUSM 28361-28371, 204: MUSM 45650, 209: MUSM 2835328360, 210: MUSM 28372-28379, 212: MUSM 44906-45649, 213: MUSM 28386-28387, 217: MUSM 28388-28389, 218: MUSM 31761-31763, 220: MUSM 28390, 221: TCWC 29491-29492, 223: MUSM 31772, 233: MUSM 28306-28319, 234: MUSM 28298-28305, 235: MUSM 28320-28329, 236: MUSM 31701-31705, 239: MUSM 31671, 241: MUSM 28416-28419, 242: MUSM 31764, 243: MUSM 31673, 245: MUSM 31672, 246: MUSM 31760, 247: MUSM 31757-31758, 248: MUSM 31759, 249: MUSM 31674-31677, 250: MUSM 28286-28290, 251: MUSM 28253-28257, 252: Hice et al. 2004, Ramos-Rodríguez et al. 2018, 253: MUSM 28275-28285, 260: Díaz 2011, 261: MUSM 31769-31771, 263: MUSM 31767-31768, 265: MUSM 28265-28269, 266: MUSM 28258-28264, 270: MUSM 28341-28352, 273: MUSM 2829128293, 274: MUSM 26571, 276: MUSM 28381-28384, 277: MUSM 31713, 279: MUSM 31714, 280: MUSM 31683-31687, 281: MUSM 31678-31680, 283: MUSM 31681-31682, 285: MUSM 31688-31700, 286: MUSM 31670 287: MUSM 31722-31725, 288: MUSM 31718-31721, 292: MUSM 3174631756, 293: MUSM 31726-31742, 294: MUSM 31716-31717, 295: MUSM 31706-31710, 296: MUSM 31743-31754, 308: MUSM 31666, 309: MUSM 31619-31622, 310: MUSM 31623-31627, 312: MUSM 31628-31642, 313: MUSM 31618, 314: MUSM 31643-31652, 315: MUSM 31663-31665, 316: Vásquez Újina 2019, 317: MUSM 26529-26532, 318: MUSM 31653-31662, 320: Ramos et al. 2017, 322: MUSM 31715, 323: MUSM 31776: MUSM 31777, 324: MUSM 21246, 328: MUSM 31667, MUSM 31668-31669, 329: Wilson, Ascorra \& Solari 1996, 335: MUSM 9421, 436, 437: Bravo \& Ríos 2007. Putumayo.- 23: Montenegro \& Moya 2011, 38: Montenegro \& Moya 2011. Requena.- 355: MUSM 841-859, 357: Wilson, Ascorra \& Solari 1996, 374: Voss et al. 2016, 394: Medina et al. 2015, 395: Medina et al. 2015, 397: Medina et al. 2015, 399: Medina et al. 2015, 407: MUSM 20444-20446, 440-442: Jorge \& Velazco 2006. Ucayali.- 420: MUSM 38288.

\section{Rhynchonycteris naso (Wied-Neuwied, 1820)}

Alto Amazonas.- 369: FMNH 19968. Loreto.- 337: KU 140185, KU 140186, KU 140189, 350: Toffoli et al. 2018, 361: MUSM 1083, MUSM 1084, 365: FMNH 122827-122829. Mariscal Ramón Castilla.- 44: FMNH 104861-104863, 326: FMNH 19960-19967, 427: Escobedo 2003, 430: Montenegro \& Escobedo 2004. Maynas.- 1: MUSM 20975-20977, 2: AMNH 71496-71500, 8: AMNH 71649-71655, 40: MUSM 6745, 41, 50: Wilson, Ascorra \& Solari 1996, 66 : AMNH 73445-73459, 80: MUSM 4355, 85: Díaz \& Linares García 2012, 106: FMNH 87003, FMNH 87004, 122: MUSM 30873, 156: MUSM 27150, MUSM 27151, 166: MUSM 30881, 172: MUSM 30874-30880, 181: MUSM 30882, 184: Díaz \& Linares García 2012, 185: FMNH 123030, 189: MUSM 27152, 191: MUSM 27157-27162, 205: MUSM 30869-30872, 212: MUSM 44835, 238: MUSM 27168, 252: Hice et al. 2004, 303: MUSM 30868, 429: Montenegro \& Escobedo 2004, 432: Bravo \& Borman 2008, 433: Bravo 2010, 435: Bravo \& Ríos 2007. Putumayo.- 23: Montenegro \& Moya 2011, 434: Bravo 2010. Requena.- 374: Voss et al. 2016, 381: MUSM 47601, 443, 445: Escobedo 2015. Ucayali.- 401: AMNH 75321, AMNH 76200-76204.

\section{Saccopteryx bilineata (Temminck, 1838)}

Datem del Marañón.- 439: Castro Vergara 2012. Loreto.- 347: KU 157980 KU 157981, 361: MUSM 3084. Mariscal Ramón Castilla.- 44: FMNH 104860, 427: Escobedo 2003. Maynas.- 4: MUSM 20979, 8: AMNH 71401-71426, 31: MUSM 24413, MUSM 24414, 41: Wilson, Ascorra \& Solari 1996, 46: FMNH 87005, 50: Wilson, Ascorra \& Solari 1996, 64: AMNH 98702, AMNH 98703, 66: AMNH 73432-73444, AMNH 74008-AMNH 74011, 77: MUSM 30885, MUSM 30886, 90: MUSM 27171, MUSM 27172, 91: Díaz \& Linares García 2012, 151: MUSM 27176, MUSM 27177, 182: Bardales Ordoñez 2015, 186: MUSM 30883, MUSM 30884, 190: MUSM 30906, MUSM 30907, 191: MUSM 27178, 231: Wilson, Ascorra \& Solari 1996, 247: MUSM 30903, MUSM 30904, 248: MUSM 30905, 251: MUSM 27169, 252: Hice et al. 2004, 259: MUSM 30891, 268: MUSM 30908, 269: MUSM 30893-30896, 271: MUSM
27170, 273: Díaz \& Linares García 2012, 277: MUSM 30898-30900, 286: MUSM 30892, 291: MUSM 30902, 294: MUSM 30901, 295: MUSM 30897, 306: MUSM 30890, 311: MUSM 30887-30889, 317: MUSM 26521, 320: Ramos et al. 2017, 329: MUSM 20980, 429: Montenegro \& Escobedo 2004. Requena.- 357: Ascorra et al. 1993, 374: Voss et al. 2016, 395, 397: Medina et al. 2015, 400: AMNH 67228, AMNH 67229. Ucayali.- 401: AMNH 7618676199, AMNH 76258, AMNH 76259, 405: MUSM 17975, MUSM 17982, MUSM 17993, MUSM 17995, MUSM 18026, 406: AMNH 98718-98721.

Saccopteryx canescens Thomas, 1901

Maynas.- 80: MUSM 4357, MUSM 4358

\section{Saccopteryx leptura (Schreber, 1774)}

Loreto.- 14: KU 157982, KU 157983. Maynas.- 4: MUSM 20981, 8: AMNH 71638, 41: Wilson, Ascorra \& Solari 1996, 50: Wilson, Ascorra \& Solari 1996, 77: MUSM 30910, 78: MUSM 4354, 80: MUSM 4206, 172: MUSM 30916, 186: MUSM 30909, 191: MUSM 27182, 247: MUSM 30915, 252: Hice et al. 2004, 253: MUSM 27180, 265: MUSM 27179, 268: MUSM 30917, 273: MUSM 27181, 277: MUSM 30913, 283: MUSM 30911, MUSM 30912, 296: MUSM 30914, 320: Ramos et al. 2017, 329: Wilson, Ascorra \& Solari 1996. Requena.- 355: Ascorra et al. 1993, 357: Wilson, Ascorra \& Solari 1996, 374: Voss et al. 2016, 399: Medina et al. 2015, 444: Escobedo 2015. Ucayali.- 401: AMNH 75320, 418: AMNH 98788, AMNH 99315.

Sphaeronycteris toxophyllum Peters, 1882

Loreto.- 11: MUSM 41669, 12: MUSM 41624, 325: FMNH 122939, FMNH 122940. Mariscal Ramón Castilla.- 47: Cabrera 1958. Maynas.- 1: MUSM 21265, 77, 127: Angulo \& Díaz 2004, 212: MUSM 44908. Ucayali.- 401: AMNH 76251

\section{Sturnira giannae Velazco y Patterson, 2019}

Alto Amazonas.- 387: Patterson \& López Wong 2014, 391: MUSM 48163. Datem del Marañón.- 9: MUSM 47302, 301: MUSM 43163-43165, 371: MUSM 43166. Loreto.- 10: MUSM 41747-41751, 11: MUSM 41670, 12: MUSM 41625, 14: KU 158122-158127, 68: MUSM 48255, 208: MUSM 48253, MUSM 48254, 337: KU 140176, 338: MUSM 16387-16393, 345: MUSM 47653, 350: Toffoli et al. 2018, 365: FMNH 122941, FMNH 122942. Mariscal Ramón Castilla.- 44: FMNH 104870, 84: FMNH 89090. Maynas.1: MUSM 21269, 3: MUSM 21267, MUSM 21268, 8: AMNH 71691-71695, 46: FMNH 87055-87057, 50: Wilson, Ascorra \& Solari 1996, 62: MUSM 33000-33002, 75: FMNH 87058, 76: MUSM 33003, 83: MUSM 29744, 90: MUSM 29679, 150: MUSM 33060, 151: MUSM 29709, 158: MUSM 32961, 169: MUSM 29752, 173: MUSM 33059, 178: MUSM 29707, MUSM 29708, 179: MUSM 33067, MUSM 33068, 180: MUSM 29772, 181: MUSM 33065, MUSM 33066, 183: MUSM 33004, 187: MUSM 29773-29775, 188: MUSM 29765-29771, 191: MUSM 29739-29741, 195: MUSM 29743, 197: MUSM 33064, 199: MUSM 29742, 202: MUSM 29747, MUSM 29748, 204: MUSM 44910, MUSM 44911, MUSM 45652-45654, 210: MUSM 29749, 211: MUSM 29753-29758, 212: MUSM 45651, 213: MUSM 29759, MUSM 29760, 216: MUSM 33048, 220: MUSM 29761-29764, 223: MUSM 33063, 231: Wilson, Ascorra \& Solari 1996, 232: MUSM 32963, 233: MUSM 29721-29724, 234: MUSM 29710-29720, 235: MUSM 29725-29738, 236: MUSM 32987-32995, 239: MUSM 32964, 241: MUSM 29780-29787, 242: MUSM 33050-33058, 244: MUSM 32967, 247: MUSM 33042-33047, 249: MUSM 32965, MUSM 32966, 250: MUSM 29690-29701, 251: MUSM 29669-29671, 252: Hice et al. 2004, 253: MUSM 29680-29689, 255: MUSM 16496, MUSM 16497, 260: Díaz 2011, 265: MUSM 29674-29677, 266: MUSM 29672, MUSM 29673, 267: MUSM 33061, 268: MUSM 33062, 270: MUSM 29745, MUSM 29746, 271 MUSM 29678, 273: MUSM 29702-29706, 275: MUSM 33049, 276: MUSM 29750, MUSM 29751, 282: MUSM 32968-32972, 285: MUSM 32973-32986, 287: MUSM 33014, MUSM 33015, 288: MUSM 33005-33013, 292: MUSM 33036-33041, 293: MUSM 33016-33021, 295: MUSM 32996-32999, 296: MUSM 33022-33025, MUSM 33027-33035, MUSM 39026, 305: MUSM 32962, 309: MUSM 32956-32958, 314: MUSM 32959, 315: MUSM 32960, 320: Ramos et al. 2017, 335: MUSM 9433, 437: Bravo \& Ríos 2007. Putumayo.- 23: Montenegro \& Moya 2011. Requena.- 355: MUSM 5922-5925, 357: Wilson, Ascorra \& Solari 1996, 397: Medina et al. 2015. Ucayali.- 402: MUSM 704, 424: Pacheco \& Arias 2001.

Sturnira magna de la Torre 1966

Alto Amazonas.- 386: Patterson \& López Wong 2014. Datem del Marañón.- 256: FMNH 89087, 387: Patterson \& López Wong 2014. Loreto.- 14: KU 158128, 20: MUSM 41626, 21: MUSM 41753, 22: MUSM 41752, 338: MUSM 16394, MUSM 16395. Mariscal Ramón Castilla.- 428: Escobedo 2003. Maynas.- 3: MUSM 21270, MUSM 21271, 41: Wilson, Ascorra \& Solari 1996, 55: LSUMZ 28260-28262, LSUMZ 28288, LSUMZ 28289, 58: MUSM 37667, 
75: FMNH 87059-87062, 233: MUSM 29792-29794, 234: MUSM 29791, 236: MUSM 33079, MUSM 33080, 240: TCWC 27474, 242: MUSM 33095, 247: MUSM 33090-33092, 251: MUSM 29788, 252: Hice et al. 2004, 253: MUSM 29789, 272: MUSM 33094, 273: MUSM 29790, 275: MUSM 33093 276: MUSM 29795, 280: MUSM 33075, MUSM 33076, 282: MUSM 33074, 285: MUSM 33077, MUSM 33078, 288: MUSM 33085, 290: MUSM 33084 291: MUSM 33083, 293: MUSM 33086-33088, 295: MUSM 33081, 296: MUSM 33089, 309: MUSM 33069, 312: MUSM 33070, 315: MUSM 33072, MUSM 33073, 316: Vásquez Újina 2019, 318: MUSM 33071, 320: Ramos et al. 2017, 323: MUSM 33096, 332: MUSM 33082, 429: Montenegro \& Escobedo 2004. Putumayo.- 15: Montenegro \& Moya 2011. Requena.- 354: MUSM 5496, 357: Wilson, Ascorra \& Solari 1996, 374: Velazco \& Patterson 2013, 394, 398, 399: Medina et al. 2015. Ucayali.- 402: MUSM 698, 409: MUSM 5253, 412: MUSM 20447, 420: MUSM 38289.

Sturnira oporaphilum (Tschudi, 1844)

Ucayali.- 415: Pacheco \& Arias 2001

\section{Sturnira tildae de la Torre, 1959}

Datem del Marañón.- 342: MUSM 38930. Loreto.- 14: KU 158131, KU 158132, 208: MUSM 48256, 347: KU 158129, KU 158130, 350: Toffoli et al. 2018, 361: MUSM 3996-3998. Loreto.- 22: MUSM 41755, 48: MUSM 41754 Maynas.- 1: MUSM 21279-21292, 3: MUSM 21273-21278, 4: MUSM 21272, 76: MUSM 33132, 171: MUSM 33141-33144, 178: MUSM 29813, MUSM 29814, 180: MUSM 29830, 182: Bardales Ordoñez 2015, 186: MUSM 33099, 187: MUSM 29831, MUSM 29832, 191: MUSM 29821-29823, 197: MUSM 33145, 203: MUSM 33136, 204: MUSM 44912-44914, MUSM 45658, 209: MUSM 29825, 212: MUSM 45655-45657, 214: MUSM 33137, 217: MUSM 29828, 220: MUSM 29829, 233: MUSM 29818, MUSM 29819, 234: MUSM 29815-29817, 235: MUSM 29820, 241: MUSM 29833-29836, 242: MUSM 33138-33140, 249: MUSM 33111-33114, 250: MUSM 29806-29812, 251: MUSM 29797, MUSM 29798, 252: Hice et al. 2004, 253: MUSM 29803MUSM 29805, 260: Díaz 2011, 265: MUSM 29799-29802, 270: MUSM 29824, 276: MUSM 29826, MUSM 29827, 282: MUSM 33115-33120, 285: MUSM 33121-33131, 288: MUSM 33133, 293: MUSM 33134, 296: MUSM 33135, 309: MUSM 33100, 312: MUSM 33101-33104, 314: MUSM 3310533110, 316: Vásquez Újina 2019, 320: Ramos et al. 2017, 429: Montenegro \& Escobedo 2004. Putumayo.- 23: Montenegro \& Moya 2011. Requena.355: MUSM 5921, MUSM 5926, 357: Wilson, Ascorra \& Solari 1996, 395: Medina et al. 2015. Ucayali.- 405: MUSM 17928, MUSM 17934, MUSM 18001, 423: MUSM 38290.

Thyroptera discifera (Lichtenstein y Peters, 1855)

Mariscal Ramón Castilla.- 44: FMNH 95907. Maynas.- 141: O. Thomas 1928d. Requena.- 357: Wilson, Ascorra \& Solari 1996.

Thyroptera lavali Pine, 1993

Mariscal Ramón Castilla.- 327: Velazco et al. 2014. Maynas.- 180: Díaz 2011, 186: MUSM 33344, 272: MUSM 33345, MUSM 33346. Requena.355: Solari et al. 2004.

Thyroptera tricolor Spix, 1822

Datem del Marañón.- 256: FMNH 89113-89116. Mariscal Ramón Castilla. 44: Velazco et al. 2014, 289: FMNH 89166-89168, 327: Velazco et al. 2014 Maynas.- 8: AMNH 71647, AMNH 71648, 50: Wilson, Ascorra \& Solari 1996, 61: AMNH 74376, AMNH 74377, 70: Velazco et al. 2014, 210: MUSM 29959, 231: Wilson, Ascorra \& Solari 1996, 232: MUSM 33348, MUSM 33349, 248: MUSM 33350, 252: Hice et al. 2004, 263: MUSM 33351, 311: MUSM 33347, 320: Ramos et al. 2017. Requena.- 374: Velazco et al. 2014, 441: Jorge \& Velazco 2006.

Thyroptera wynneae Velazco, Gregorin, Voss y Simmons, 2014 Requena.- 357: Velazco et al. 2014.

Tonatia maresi Williams, Willig y Reid, 1995

Alto Amazonas.- 387: Patterson \& López Wong 2014. Loreto.- 350: Toffoli et al. 2018. Maynas.- 1: MUSM 21293, MUSM 21294, 40: MUSM 21179, 41, 50: Wilson, Ascorra \& Solari 1996, 59: MUSM 26578, 188: MUSM 28735, 218: MUSM 32281, 226: MUSM 32280, 227: MUSM 32282, MUSM 32283 247: MUSM 32279, 251: MUSM 28731, 252: Hice et al. 2004, 261: MUSM 32284, 265: MUSM 28732, 269: MUSM 32266, 270: MUSM 28734, 273: MUSM 28733, 274: MUSM 26538, MUSM 26539, 280: MUSM 32268, 283 MUSM 32267, 285: MUSM 32269, MUSM 32270, 287: MUSM 32276, 291: MUSM 32275, 292: MUSM 32277, 295: MUSM 32271-32273, 296: MUSM 32278, 309: MUSM 32260, 312: MUSM 32261, 316: Vásquez Újina 2019, 318: MUSM 32262, MUSM 32263, 320: Ramos et al. 2017, 323: MUSM
32285, 328: MUSM 32264, MUSM 32265, 332: MUSM 32274, 437: Bravo \& Ríos 2007. Requena.- 357: Ascorra et al. 1993, 374: Voss et al. 2016, 392 MUSM 47655, MUSM 47656, 442: Jorge \& Velazco 2006.

\section{Trachops cirrhosus (Spix, 1823)}

Datem del Marañón.- 256: FMNH 89026-89030, 360: MUSM 43167, MUSM 43168, Loreto.- 14: KU 158149-158151, 338: MUSM 16400, 347: KU 158146158148, 350: Toffoli et al. 2018, 359: MUSM 47659. Mariscal Ramón CastiIla.- 47: FMNH 29450, 327: FMNH 89031-89035, 426, 427: Escobedo 2003. Maynas.- 1: MUSM 21296-21301, 3: MUSM 21295, 31: MUSM 24427, 39: MUSM 27078, 40: MUSM 21178, 41, 50: Wilson, Ascorra \& Solari 1996, 55 LSUMZ 28281, 28282, 70: AMNH 74026, 186: MUSM 32287, 188: MUSM 28737, 204: MUSM 45659, 230: MUSM 32301, 231: Wilson, Ascorra \& Solari 1996, 251: MUSM 28736, 252: Hice et al. 2004, 263: MUSM 32302, 274: MUSM 26540, 277: MUSM 32298, 278: MUSM 32297, 286: MUSM 32295, MUSM 32296, 288: MUSM 32300, 308: MUSM 32293, MUSM 32294, 310: MUSM 32290, 311: MUSM 32291, 313: MUSM 32288, MUSM 32289, 315 MUSM 32292, 316: Vásquez Újina 2019, 317: MUSM 26541, 329: Wilson, Ascorra \& Solari 1996, 332: MUSM 32299. Putumayo.- 30: Montenegro \& Moya 2011, 431: Montenegro \& Escobedo 2004. Requena.- 357: Ascorra et al. 1993, 374: Voss et al. 2016, 443, 445: Escobedo 2015. Ucayali.- 401: AMNH 76162-76164.

\section{Trinycteris nicefori (Sanborn, 1949)}

Alto Amazonas.- 388: Patterson \& López Wong 2014. Datem del Marañón. 342: MUSM 38931. Maynas.- 41, 50: Wilson, Ascorra \& Solari 1996, 66 : Pirlot 1968, 171: MUSM 32308, MUSM 32309, 181: MUSM 32310, MUSM 32311, 209: MUSM 28739, 222: MUSM 32304, 248: MUSM 32307, 251: MUSM 28738, MUSM 28740, 252: Hice et al. 2004, 258: MUSM 32305, 269: MUSM 31996, 280: MUSM 32303, 293: MUSM 31998, MUSM 32306, 320: Ramos et al. 2017, 323: MUSM 32312, 329: MUSM 21304, MUSM 21305 , 330: MUSM 21302, MUSM 21303. Requena.- 357: Ascorra et al. 1993, 374: Voss et al. 2016.

Uroderma bilobatum Peters, 1866

Alto Amazonas.- 388: Patterson \& López Wong 2014, 391: MUSM 48164 Datem del Marañón.- 25: MUSM 39754, 67: MUSM 25874, MUSM 25875 256: FMNH 89081, 343: MUSM 16401, 383: Patterson \& López Wong 2014. Loreto.- 11: MUSM 41671, MUSM 41672, 208: MUSM 48257, 325: FMNH 122890-122901, KU 140204-140221, KU 140235, KU 140248, KU 140249, KU 140257-140259, KU 140265, KU 140266, 344: Toffoli et al. 2018, 345: MUSM 47660, 347: KU 158152, 348: MUSM 47661, 361: MUSM 1026, MUSM 4151, MUSM 4152, 365: FMNH 122883-122889, 366: FMNH 87037, FMNH 87083. Mariscal Ramón Castilla.- 327: FMNH 153623, FMNH 89084, FMNH 89086, FMNH 89130, FMNH 89131, 426: Escobedo 2003. Maynas.- 1: MUSM 21310-21319, 4: MUSM 21307-21309, 40: MUSM 21306, MUSM 6749, 41: Wilson, Ascorra \& Solari 1996, 46: FMNH 87036, FMNH 87082, 50: Wilson, Ascorra \& Solari 1996, 55: LSUMZ 28265-28267, LSUMZ 28274-28276, 63: LSUMZ 28433, 66: AMNH 73504, 71: MUSM 27083, 75: FMNH 87038-87043, FMNH 87084, FMNH 89127-89129, 76: MUSM 33186, MUSM 33187, 77: MUSM 33146, 80: MUSM 4364, 83: MUSM 29882, MUSM 29883, 87: TCWC 27487, 90: MUSM 29845-29847, 139: MUSM 29898, 149: MUSM 33188, 151: MUSM 29862-29872, 158: MUSM 33159, 163: MUSM 29903, 172: MUSM 33206, 179: MUSM 33213-33216, 180: MUSM 29900, 181: MUSM 33210-33212, 183: MUSM 33189-33191, 184: MUSM 29848, 188: MUSM 29899, 189: MUSM 29844, 195: MUSM 29881, 196: MUSM 29852-29861, 197: MUSM 33208, MUSM 33209, 198: TCWC 27484, 199: MUSM 29879 MUSM 29880, 202: MUSM 29890, MUSM 29891, 204: MUSM 45661, 207 MUSM 44915, MUSM 44916, MUSM 44918, MUSM 44919, 209: MUSM 29887-29889, 211: MUSM 29892, MUSM 29893, 212: MUSM 44917, MUSM 45660, 217: MUSM 29894, 220: MUSM 29895-29897, 221: TCWC 29493-29504, 222: MUSM 33169, 223: MUSM 33207, 225: MUSM 43014, 231: Wilson, Ascorra \& Solari 1996, 232: MUSM 33170, 233: MUSM 29875 234: MUSM 29873, MUSM 29874, 235: MUSM 29876-29878, 236: MUSM 33181, 238: MUSM 29904, 239: MUSM 33171, 240: ROM 77319, ROM 77320, TCWC 27475-27483, TCWC 27485, TCWC 27486, 242: MUSM 33205, 243: MUSM 33172, 247: MUSM 33200-33202, 249: MUSM 33173, MUSM 33174, 250: MUSM 29849, 251: MUSM 29837-29839, 252: Hice et al. 2004, 260: MUSM 29901, MUSM 29902, 265: MUSM 29843, 266: MUSM 2984029842, 269: MUSM 33175, 270: MUSM 29884-29886, 273: MUSM 29850, MUSM 29851, 275: MUSM 33203, MUSM 33204, 280: MUSM 33177, 282: MUSM 33176, 285: MUSM 33178-33180, 286: MUSM 33168, 292: MUSM 33196, MUSM 33197, MUSM 33199, 293: MUSM 33193-33195, 295: MUSM 33182-33185, 296: MUSM 33198, 305: MUSM 33160-33163, 306: MUSM 33164, 308: MUSM 33165, 309: MUSM 33147-33153, 312: MUSM 33154, 314: MUSM 33155, MUSM 33156, 315: MUSM 33158, 316: Vásquez Újina 
2019, 318: MUSM 33157, 320: Ramos et al. 2017, 322: MUSM 33192, 323: MUSM 33217, 328: MUSM 33166, MUSM 33167, 433: Bravo 2010, 437: Bravo \& Ríos 2007. Requena.- 357: Wilson, Ascorra \& Solari 1996, 367: FMNH 89082, 395, 397-399: Medina et al. 2015, 411: MUSM 20448, 441: Jorge \& Velazco 2006, 444: Escobedo 2015. Ucayali.- 405: MUSM 17923, MUSM 17937, MUSM 17960, MUSM 17970, MUSM 18019, 409: FMNH 64313, FMNH 64315, MUSM 1027, MUSM 1028, 412: MUSM 20449, 415: Pacheco \& Arias 2001.

\section{Uroderma magnirostrum Davis, 1968}

Loreto.- 348: MUSM 47662, 350: Toffoli et al. 2018. Mariscal Ramón Castilla.- 426, 428: Escobedo 2003. Maynas.- 50: Wilson, Ascorra \& Solari 1996, 55: LSUMZ 28264, LSUMZ 28272, 64: AMNH 98707: AMNH 98768 80: MUSM 4362, 81: MUSM 29924-29926, 83: MUSM 29927-29930, 90: MUSM 29910, 110: MUSM 29915, 130: MUSM 33227, 134: MUSM 29916 135: MUSM 29906, MUSM 29907, 140: MUSM 29908, 143: MUSM 29935, 146: MUSM 29909, 149: MUSM 33228-MUSM 33230, 159: MUSM 29944 169: MUSM 29937-MUSM 29939, 173: MUSM 33234, 181: MUSM 33235, MUSM 33236, 183: MUSM 33231, 184: MUSM 29911, 191: MUSM 29923 196: MUSM 29917-MUSM 29918, MUSM 29919, 202: MUSM 29931-29934, 211: MUSM 29940, 213: MUSM 29941, MUSM 29942, 220: MUSM 29943, 234: MUSM 29920-29922, 237: MUSM 33226, 247: MUSM 33233, 250: MUSM 29912-29914, 251: MUSM 29905, 252: Hice et al. 2004, 276: MUSM 29936, 282: MUSM 33225, 288: MUSM 33232, 309: MUSM 33218-33220, 312: MUSM 33221, 314: MUSM 33222, MUSM 33223, MUSM 33224, 316: Vásquez Újina 2019, 320: Ramos et al. 2017, 335: MUSM 9431. Putumayo.38: Montenegro \& Moya 2011. Requena.- 357: MUSM 5595, Wilson, Ascorra \& Solari 1996. Ucayali.- 401: AMNH 76236, AMNH 76237.

Vampyressa thyone Thomas, 1909

Alto Amazonas.- 388: Patterson \& López Wong 2014. Datem del Marañón.9: MUSM 47303, 67: MUSM 25876, 304: MUSM 16406, 342: MUSM 38932 383: Patterson \& López Wong 2014. Loreto.- 11: MUSM 41673, 13: Ruelas \& Pacheco 2015, 14: KU 158155, KU 158156, 22: MUSM 41756-41758, 29: Ruelas \& Pacheco 2015, MUSM 41627, 168: MUSM 41134, 170: MUSM 41132, MUSM 41133, 347: KU 158154, 359: MUSM 38423. Mariscal Ramón Castilla.- 84: FMNH 89069, 327: FMNH 89059-89067, FMNH 89141-89145. Maynas.- 1: MUSM 21188, MUSM 21328, 3: Ruelas \& Pacheco 2015, 8: AMNH 71675, AMNH 71680, 32: LSUMZ 28437, 40: MUSM 21324, MUSM 21325, MUSM 6748, 41, 50: Wilson, Ascorra \& Solari 1996, 63: LSUMZ 28438, LSUMZ 28439, 75: FMNH 87097-87107, FMNH 89137, FMNH 89138, 77: Ruelas \& Pacheco 2015, 179: MUSM 33284, MUSM 33285, 181: MUSM 33283, 183: MUSM 33256, 195: MUSM 29945, 198: TCWC 27521, TCWC 27522, 202: MUSM 29946, 207: MUSM 44920, MUSM 44921, 213: MUSM 29947, 216: MUSM 33267-33280, 218: MUSM 33264, MUSM 33265, 221 TCWC 29544-29565, 224: MUSM 33281, 226: MUSM 33263, 230: MUSM 33266, 236: MUSM 33254, 239: MUSM 33328, 240: ROM 77380-77382, TCWC 27523-27552, 252: Hice et al. 2004, 260: Díaz 2011, 263: MUSM 33282, 279: MUSM 33257, MUSM 33258, MUSM 33329, 280: MUSM 33252, 285: MUSM 33253, 291: MUSM 33261, MUSM 33262, 295: MUSM 33255, 300: MUSM 33259, 307: MUSM 33250, 310: MUSM 33241-33244, 312: MUSM 33245, MUSM 33246, 313: MUSM 33239, MUSM 33240, 315: MUSM 33247-33249, 329: MUSM 21185, 332: MUSM 33260, 437: Bravo
\& Ríos 2007. Putumayo.- 15: Montenegro \& Moya 2011. Requena.- 355 : MUSM 861, 357: Wilson, Ascorra \& Solari 1996, 367: FMNH 89053-89056, FMNH 89139, FMNH 89140, 394, 398, 399: Medina et al. 2015, 411: MUSM 20450, MUSM 20451, 440-442: Jorge \& Velazco 2006. Ucayali.- 405: MUSM 17921, MUSM 17996, MUSM 18007, MUSM 18009, 420: MUSM 38291, 425: Pacheco \& Arias 2001.

Vampyriscus bidens (Dobson, 1878)

Alto Amazonas.- 388: Patterson \& López Wong 2014. Datem del Marañón.17: Ruelas \& Pacheco 2015, 26: MUSM 41674, 343: Ruelas \& Pacheco 2015, 352: FMNH 29177, FMNH 29178, 387: Patterson \& López Wong 2014, 438: Castro Vergara 2012. Loreto.- 20, 22: Ruelas \& Pacheco 2015, 92: MUSM 39376, 325: FMNH 122881, FMNH 122882, KU 140203, 347: KU 158153, 382: MUSM 47663. Maynas.- 3: Ruelas \& Pacheco 2015, 8: AMNH 71656, 28: MUSM 24428, 46: FMNH 87071, FMNH 87072, FMNH 87081, 55: LSUMZ 28273, 71: MUSM 27084, 75: FMNH 87075, FMNH 87076, 179: MUSM 33324, 207: MUSM 44922-44924, 212: MUSM 44925, MUSM 45662-45666, 216: Ruelas \& Pacheco 2015, 218: MUSM 33311-33321, 221: TCWC 2950529543, 226: MUSM 33310, 230: MUSM 33323, 240: TTU 47979-47982, ROM 77376-77379, TCWC 27488-27520, 241: MUSM 29952, 251: MUSM 29949, 252: Hice et al. 2004, 260: Ruelas \& Pacheco 2015, 265: MUSM 29950, 269: Ruelas \& Pacheco 2015, 274: MUSM 26548, 280: MUSM 33301, 281: MUSM 33299, 283: MUSM 33300, 285: MUSM 33302, MUSM 33303, 287: MUSM 33305, 288: MUSM 33304, 293: MUSM 33306-33309, 309: MUSM 33287, 310: MUSM 33288-33290, 312: MUSM 33291-33293, 313: MUSM 33286, 314: MUSM 33294, 317: MUSM 26549, MUSM 26550, 318: MUSM 33295, MUSM 33296, 320: Ramos et al. 2017, 324: MUSM 21320, 329: Wilson, Ascorra \& Solari 1996, 435: Bravo \& Ríos 2007. Requena.- 440: Jorge \& Velazco 2006, 443: Escobedo 2015. Ucayali.- 405: MUSM 17980, MUSM 18015.

Vampyriscus brocki (Peterson, 1968)

Mariscal Ramón Castilla.- 428: Escobedo 2003. Maynas.- 90: Ruelas \& Pacheco 2015, 179: MUSM 33342, 181: Ruelas \& Pacheco 2015, 186: Ruelas \& Pacheco 2015, 188: MUSM 29957, 190, 202: Ruelas \& Pacheco 2015, 207 : MUSM 44926-44929, 212: MUSM 44930, MUSM 44931, 216: MUSM 33338, 218, 247, 251: Ruelas \& Pacheco 2015, 252: Hice et al. 2004, 263: MUSM 33340, 270: MUSM 29955, 293, 305: Ruelas \& Pacheco 2015, 316: Vásquez Újina 2019, 320: Ramos et al. 2017. Requena.- 355, 357: Ascorra et al. 1993, 394, 398, 399: Medina et al. 2015. Ucayali.- 425: Pacheco \& Arias 2001.

Vampyrodes caraccioli (Thomas, 1889)

Datem del Marañón.- 343: Velazco \& Simmons 2011, 352: Thomas 1924b, 353: Velazco \& Simmons 2011. Loreto.- 325: Velazco \& Simmons 2011 , 337: KU 140200-140202, 361: Velazco 2005, 365: Velazco \& Simmons 2011. Maynas.- 1, 40: Velazco \& Simmons 2011, 41: Wilson, Ascorra \& Solari 1996, 46: Velazco \& Simmons 2011, 252: Hice et al. 2004, 258: MUSM 33343, 320: Ramos et al. 2017. Requena.- 374: MUSM 15288, MUSM 15289, 399: Medina et al. 2015, 441: Jorge \& Velazco 2006.

Vampyrum spectrum (Linnaeus, 1758)

Maynas.- 244: MUSM 32313, MUSM 32314, 251: Díaz 2011, 263: MUSM 32316, 300: MUSM 32315. Requena.- 357: Wilson, Ascorra \& Solari 1996. 
Anexo 3. Mapas de distribución de las especies de murciélagos de Loreto, Perú.
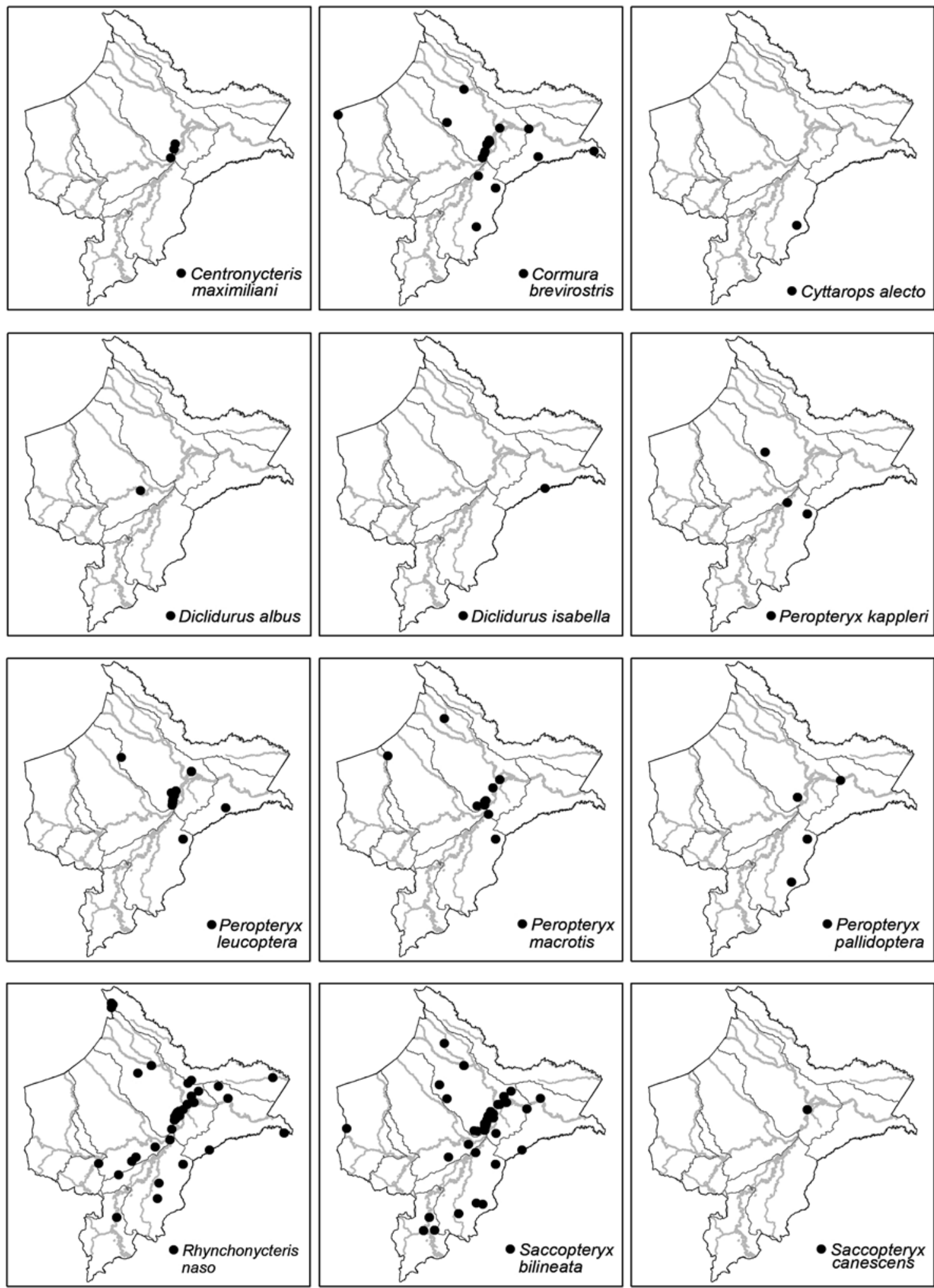

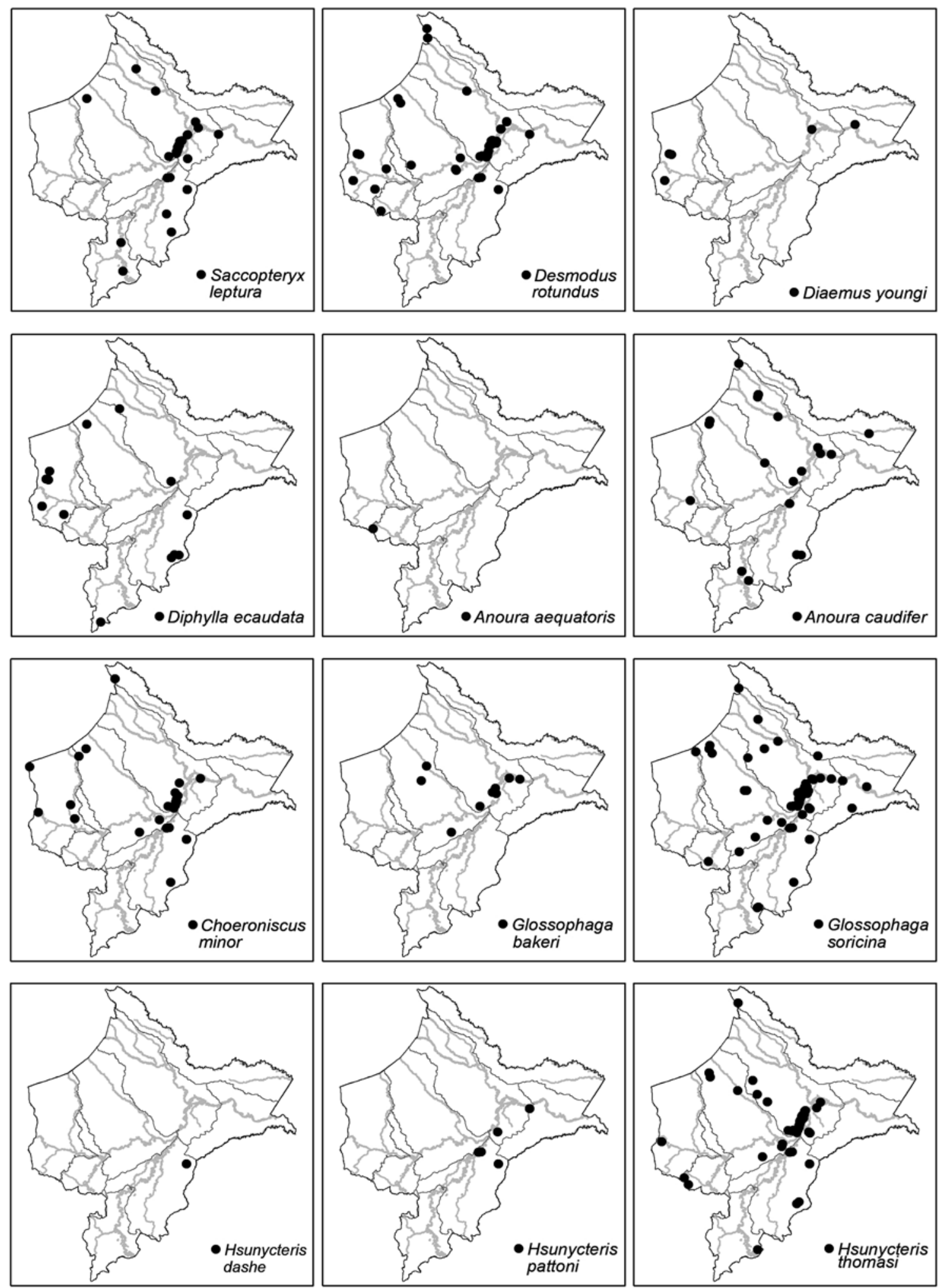

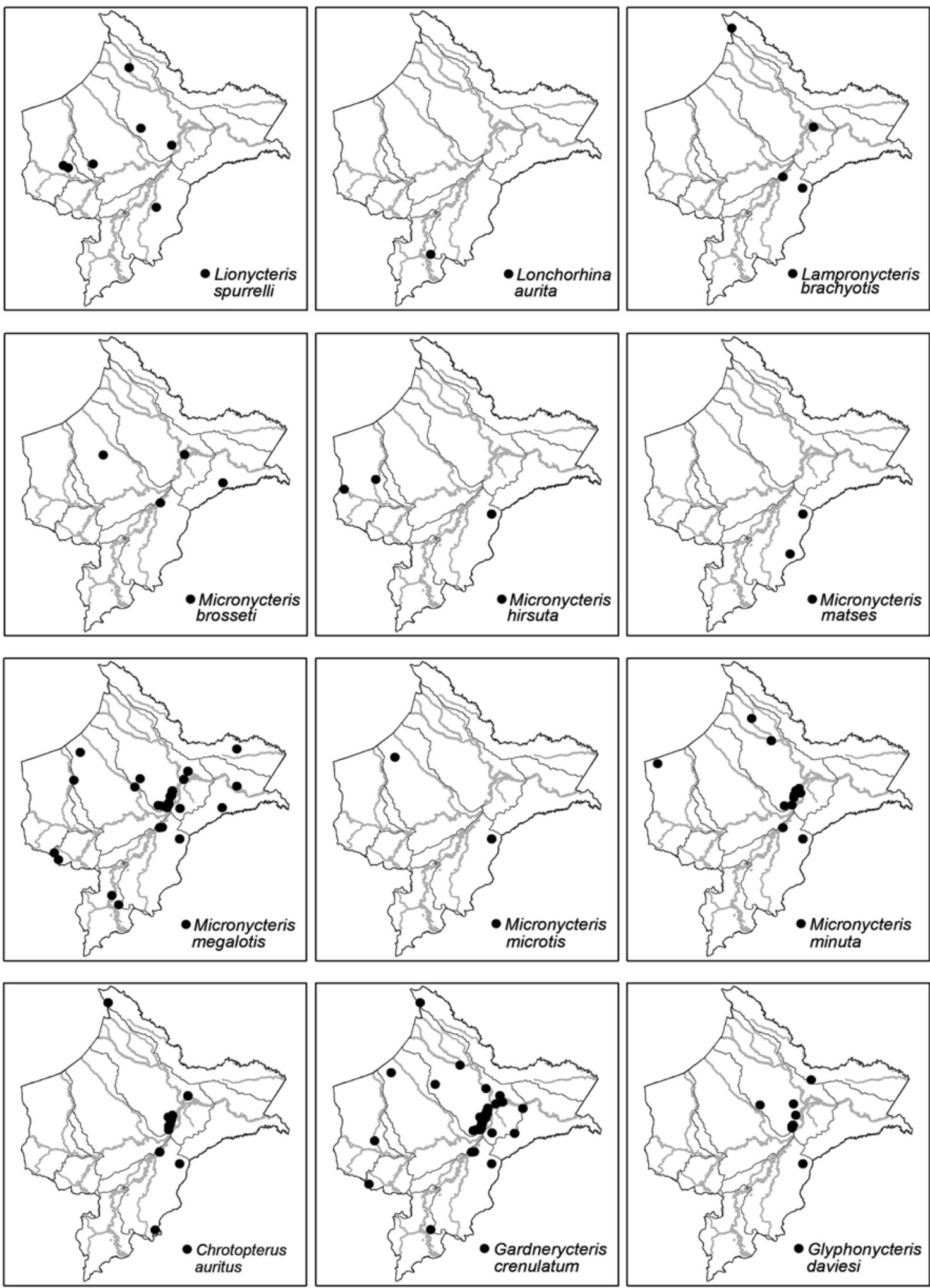

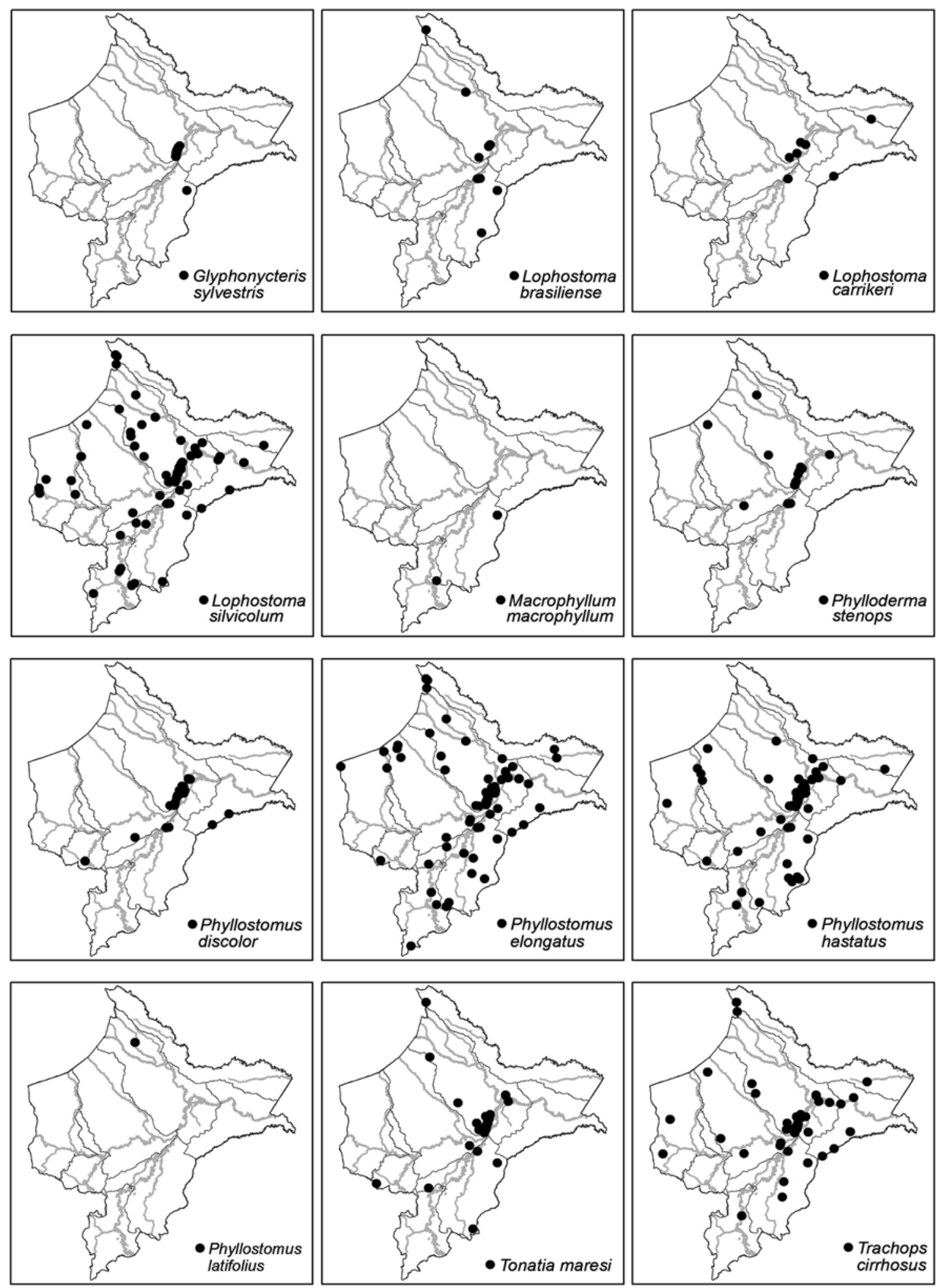

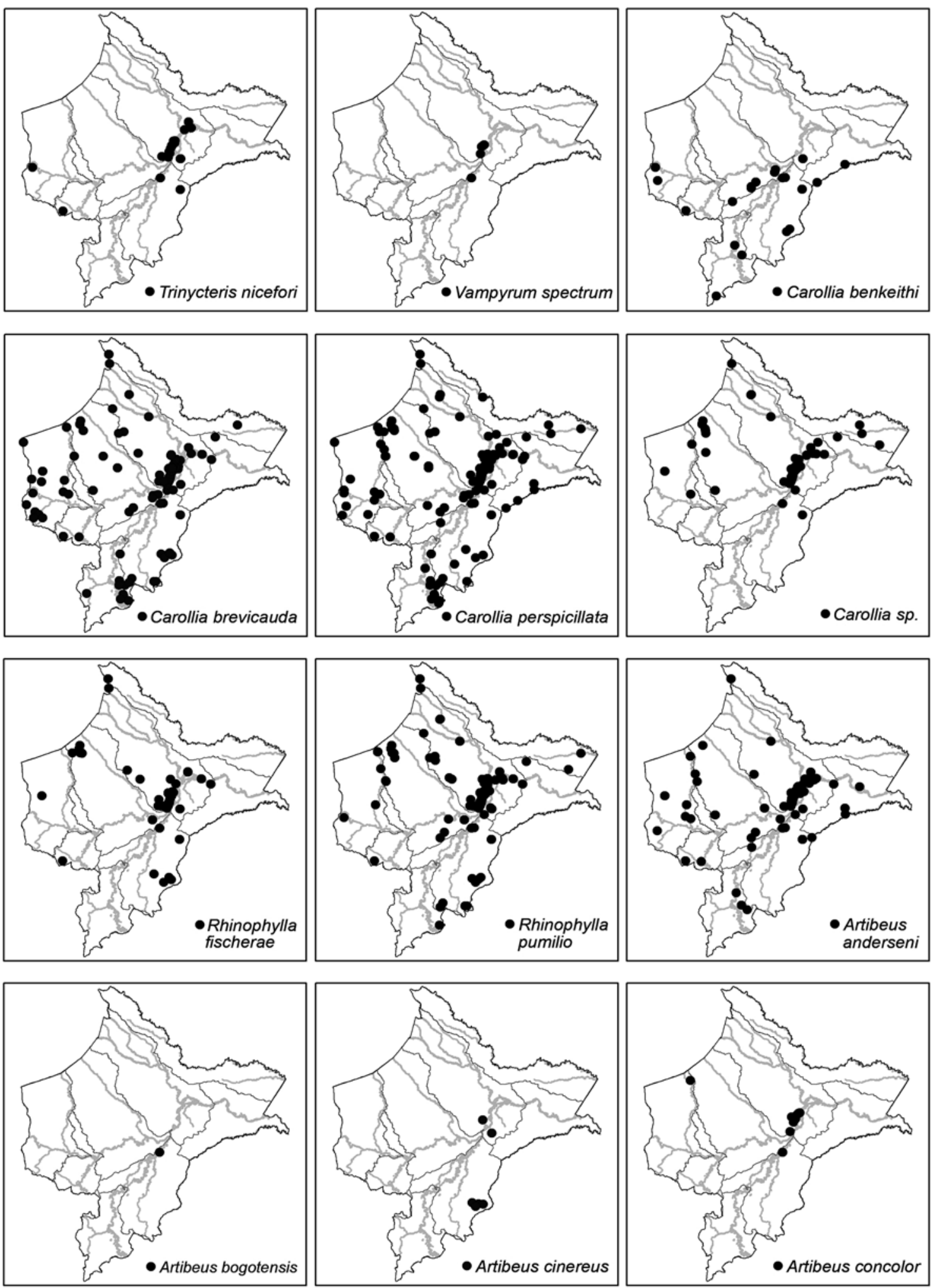

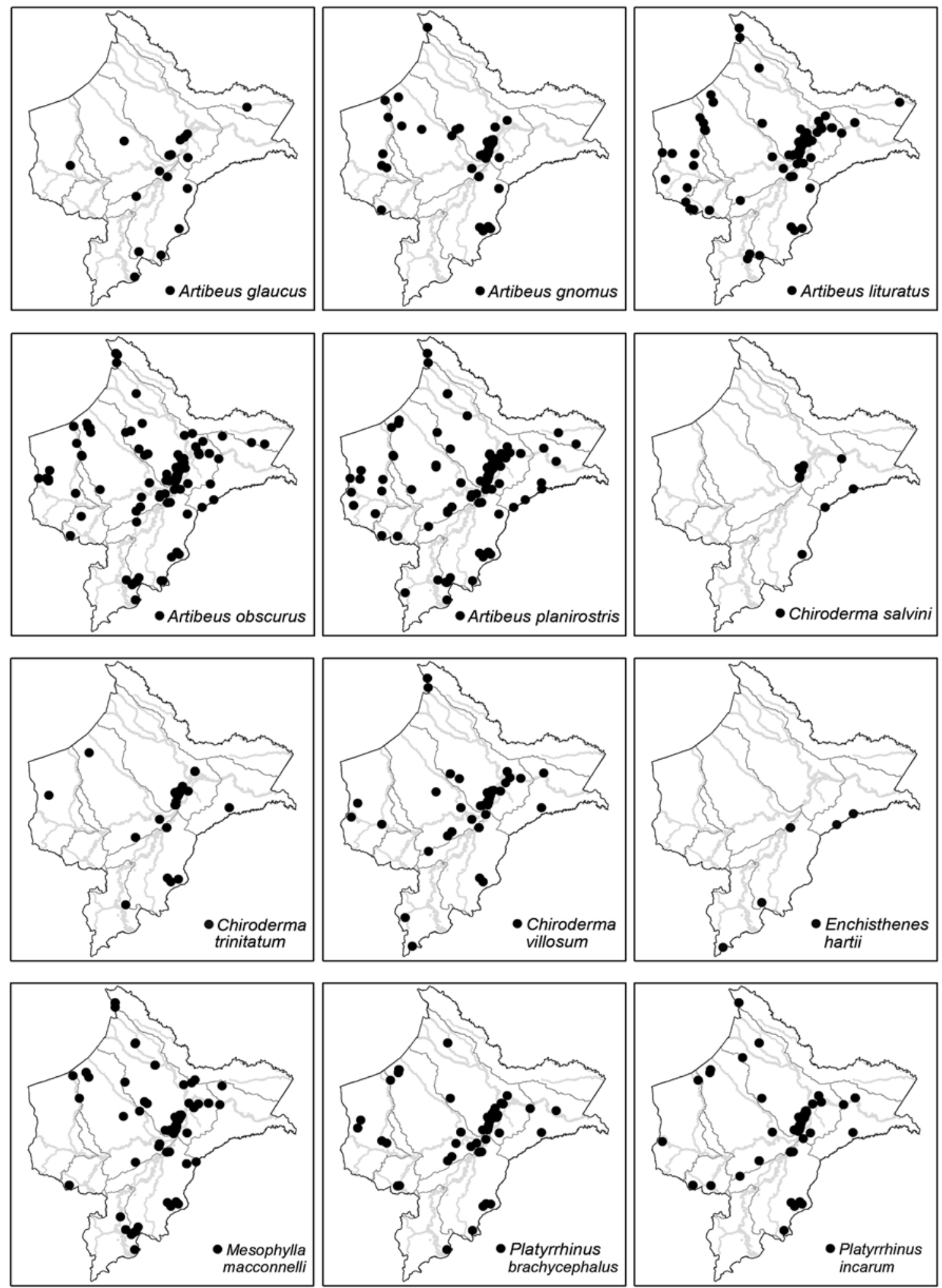

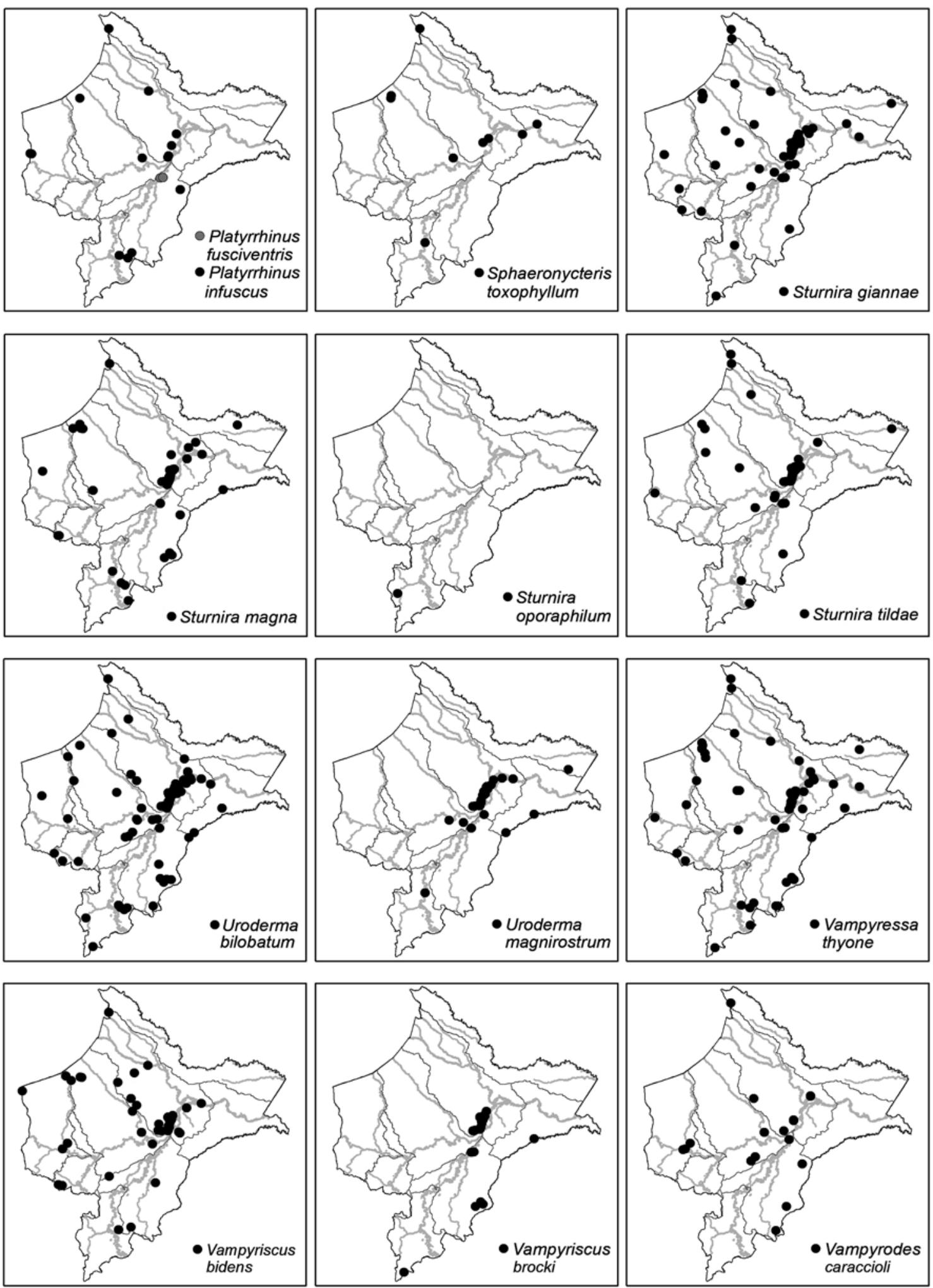

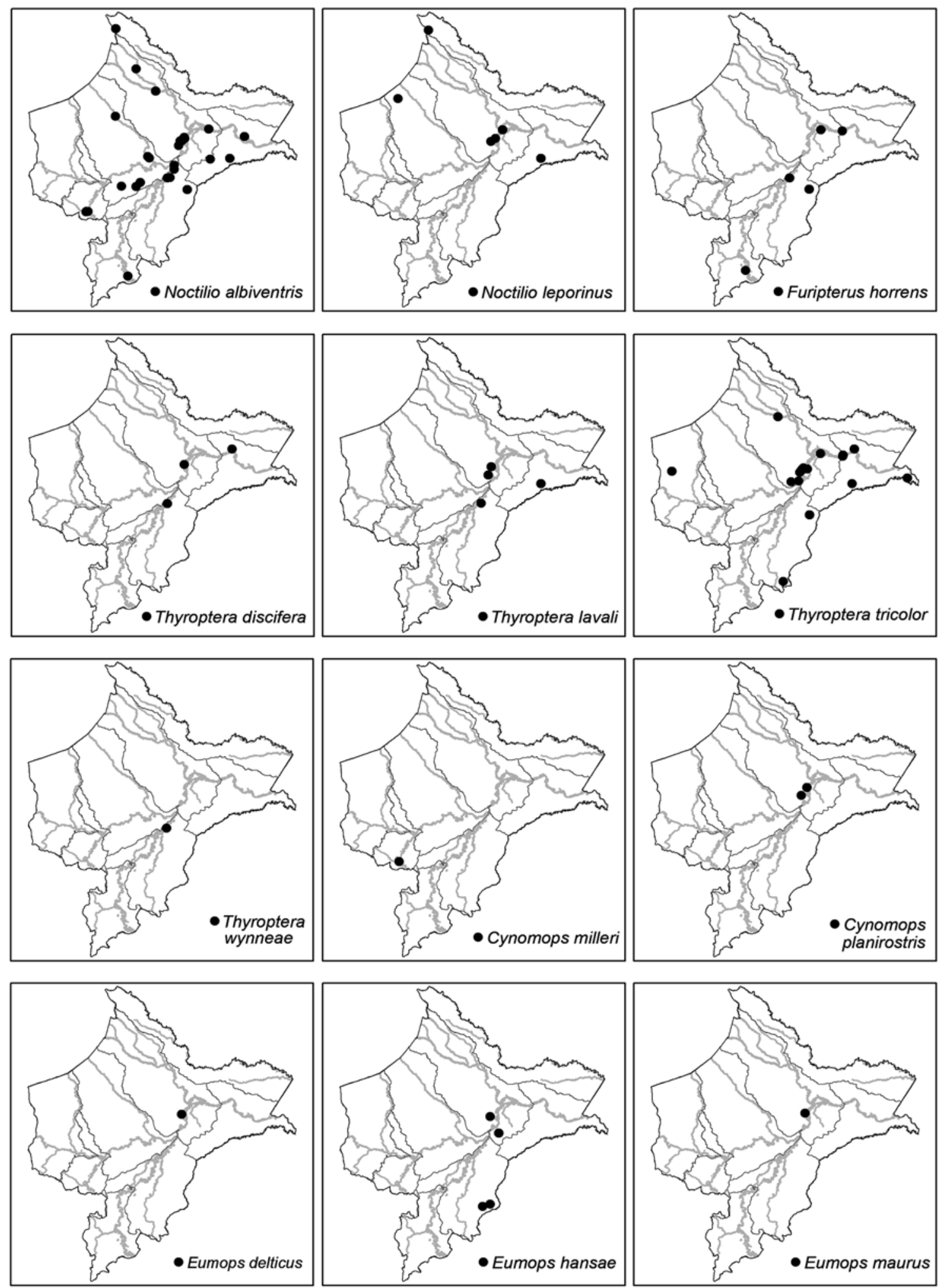

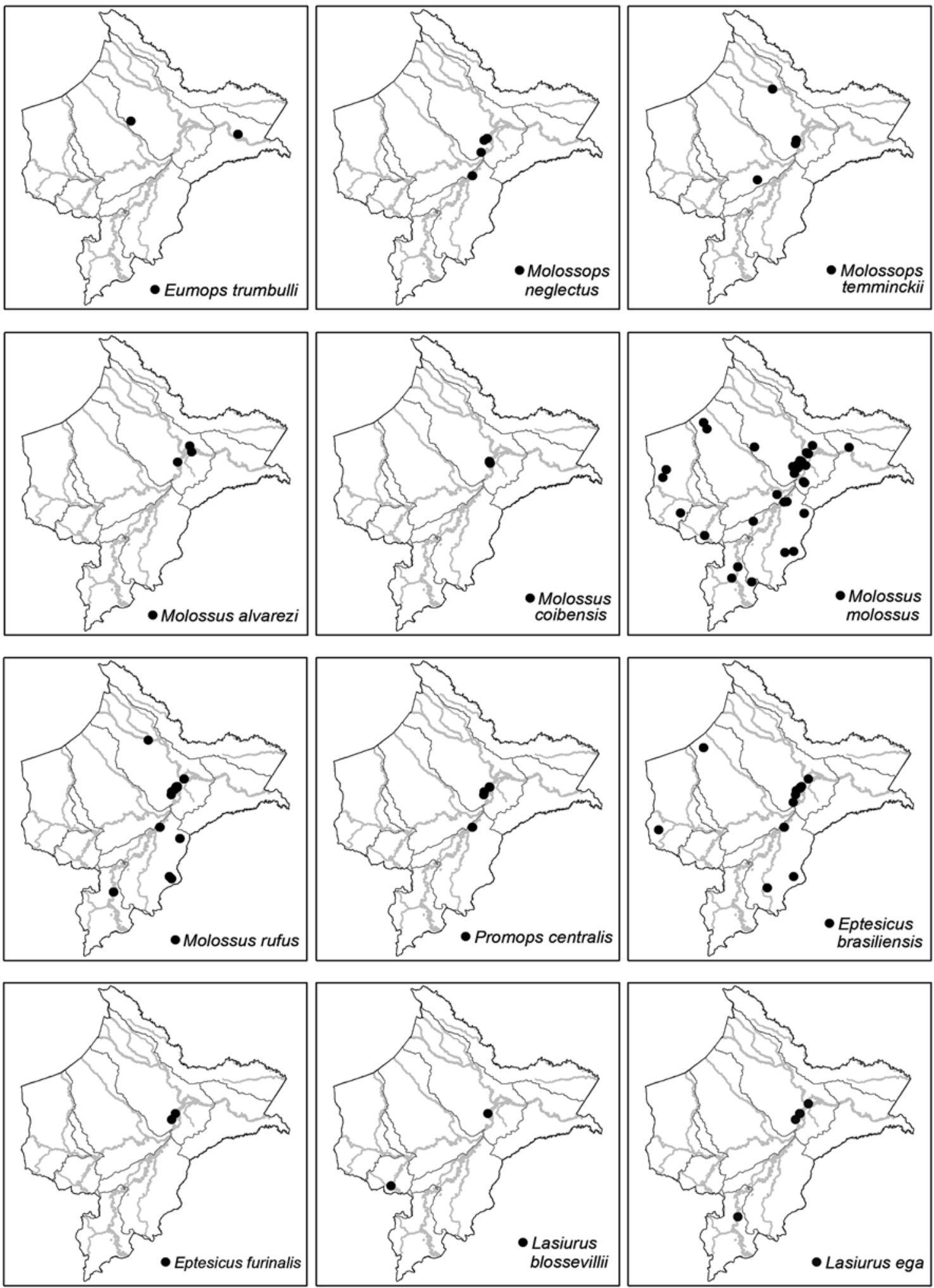

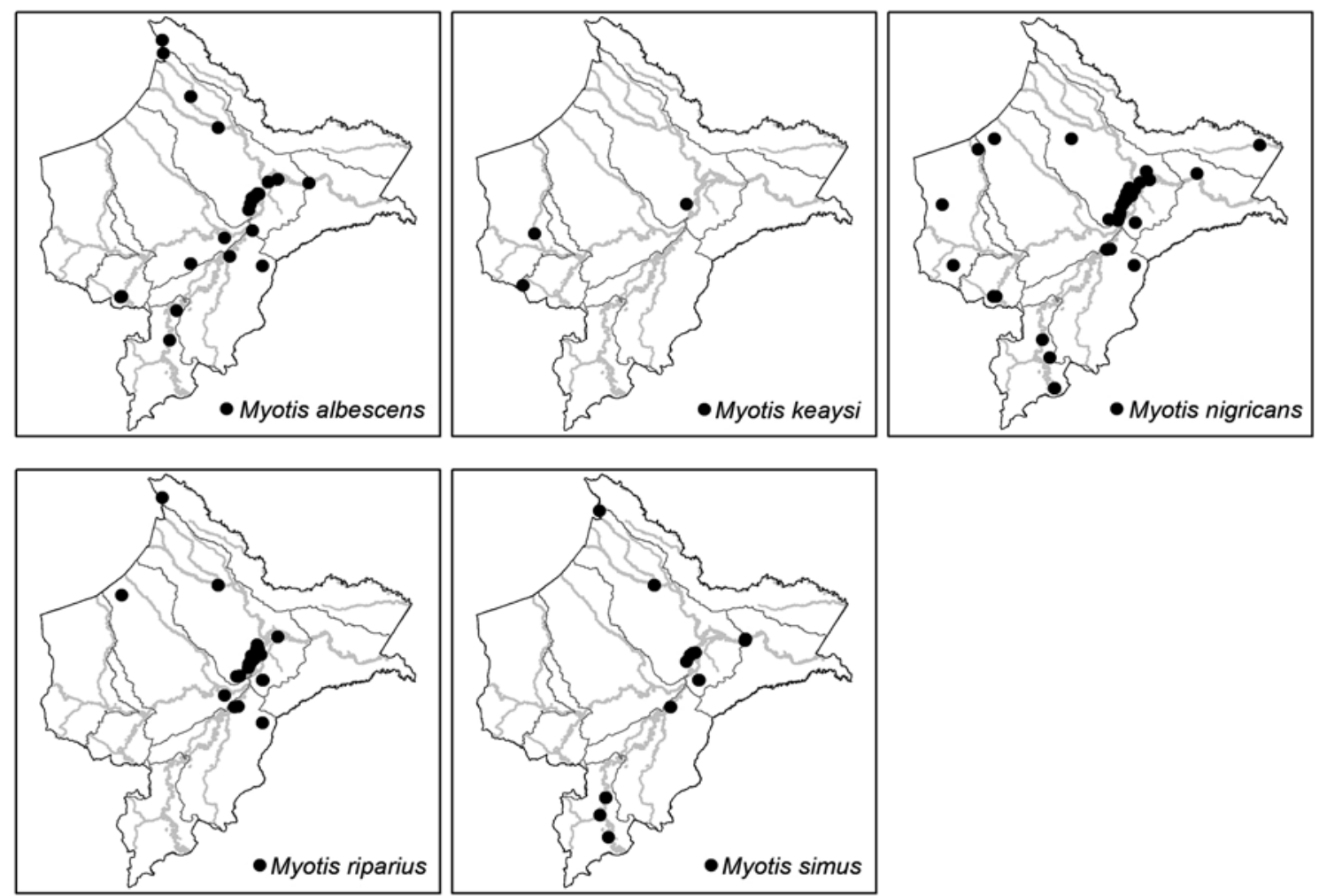